\title{
The structure-specific endonuclease complex SLX4/XPF regulates Tus/Ter-induced homologous recombination
}

Ralph Scully ( $\square$ rscully@bidmc.harvard.edu )

Beth Israel Deaconess Medical Cntr, Harvard Medical School https://orcid.org/0000-0002-5064-0175

\section{Rajula Elango}

Beth Israel Deaconess Medical Center/Harvard Medical School

\section{Arvind Panday}

Beth Israel Deaconess Medical Center/Harvard Medical School

\section{Francis Lach}

The Rockefeller Univeristy

\section{Nicholas Willis}

Beth Israel Deaconess Medical Center/Harvard Medical School

\section{Erin Duffey}

Beth Israel Deaconess Medical Center/Harvard Medical School

\section{Agata Smogorzewska}

The Rockefeller University https://orcid.org/0000-0001-6285-1562

\section{Article}

Keywords: Fanconi anemia, homologous recombination, SLX4, XPF, replication fork stalling, Tus/Ter, DSB repair, long tract gene conversion, break-induced replication

Posted Date: October 11th, 2021

DOI: https://doi.org/10.21203/rs.3.rs-904746/v1

License: (1) (1) This work is licensed under a Creative Commons Attribution 4.0 International License. Read Full License

Version of Record: A version of this preprint was published at Nature Structural \&amp; Molecular Biology on August 8th, 2022. See the published version at https://doi.org/10.1038/s41594-022-00812-9. 
The structure-specific endonuclease complex SLX4/XPF regulates Tus/Ter-induced homologous recombination

9 Rajula Elango ${ }^{1}$, Arvind Panday ${ }^{1}$, Francis P. Lach ${ }^{2}$, Nicholas A. Willis ${ }^{1}$, Erin E. Duffey ${ }^{1}$, Agata Smogorzewska ${ }^{2}$ and Ralph Scully, ${ }^{1 *}$

11

17 1. Department of Medicine, Division of Hematology-Oncology and Cancer Research Institute, 18 Beth Israel Deaconess Medical Center and Harvard Medical School, Boston, MA 02215, USA.

19 2. Laboratory of Genome Maintenance, The Rockefeller University, New York, NY 10065, 20 USA.

$21 *$. Correspondence: rscully@,bidmc.harvard.edu 


\section{Abstract}

24 Vertebrate replication forks arrested at an interstrand DNA crosslink (ICL) can engage the

25 Fanconi anemia (FA) pathway of ICL repair. The FANCP product, SLX4, binds the

26 FANCQ/XPF/ERCC4-ERCC1 endonuclease, which incises bidirectionally arrested forks to

27 'unhook' the ICL. The resulting double strand break (DSB) is repaired by homologous

28 recombination (HR). Whether this mechanism operates at replication blocks other than ICLs is

29 unknown. Here, we study the role of mammalian SLX4 in HR triggered by a site-specific,

30 chromosomal DNA-protein replication fork barrier formed by the Escherichia coli-derived

31 Tus/Ter complex. We identify an SLX4-XPF-mediated step that is required for Tus/Ter-induced

32 HR but not for HR induced by a replication-independent DSB. We additionally identify a

33 requirement for SLX4-XPF in DSB-induced 'long tract' gene conversion, a replicative HR

34 pathway related to break-induced replication. Our work suggests that Tus/Ter-induced HR

35 recapitulates the incision step of replication-coupled ICL repair, and that the full FA mechanism 36 can process DNA-protein barriers for HR.

38 Keywords: Fanconi anemia, homologous recombination, SLX4, XPF, replication fork stalling, 39 Tus/Ter, DSB repair, long tract gene conversion, break-induced replication. 
Introduction

42 DNA replication is frequently challenged by DNA template alterations that impede progression

43 of the replication fork. Replication fork stalling ('replication stress') can be triggered by specific

44 types of DNA damage or DNA repair intermediates, by unusual secondary structures in

45 undamaged DNA, by collisions with transcription complexes, or by depletion of the nucleotide

46 pool ${ }^{1-4}$. Interstrand crosslinks (ICLs) covalently bind the two parental strands, preventing

47 passage of the replicative CMG helicase. Unless the ICL can be disrupted or bypassed, it is an

48 absolute block to replication fork progression ${ }^{5-7}$. In higher eukaryotes, the Fanconi anemia (FA)

49 pathway can process the ICL-blocked fork for repair by homologous recombination (HR) ${ }^{8}$.

50 Fanconi anemia is a rare, hereditary syndrome characterized by progressive bone marrow failure,

51 developmental anomalies and cancer predisposition ${ }^{9,10}$. Cells from FA patients display

52 characteristic hypersensitivity to ICL-inducing agents, including aldehydes, which are an

53 endogenous source of both ICLs and DNA-protein crosslinks ${ }^{6,11-14}$. FA mutant cells reveal

54 elevated frequencies of ICL-induced 'chromatid-type' aberrations, reflecting a defect in

55 replication-coupled ICL repair. To date, 23 FA genes have been identified.

57 Studies of repair of ICL-containing plasmids replicating in vitro in Xenopus laevis egg extracts

58 have begun to reveal the fundamental steps of the FA pathway ${ }^{8,15}$. In this setting, FA pathway-

59 mediated repair requires stalling of two opposing replication forks at the ICL. This bidirectional

60 fork stalling triggers replisome disassembly ('fork collapse'), orchestrated by the E3 ubiquitin

61 ligase TRAIP, which polyubiquitinates CMG helicase components in trans, targeting them for

62 extraction by the p97/VCP ATPase and for proteasomal degradation ${ }^{16}$. Association of the DNA 
63 translocase and scaffolding protein FANCM and its associated proteins with the stalled fork

64 recruits the FA core complex-a large multi-subunit E3 ubiquitin ligase that monoubiquitinates

65 and thereby activates the FANCD2-FANCI heterodimer ${ }^{8,17-19}$. Monoubiquitinated FANCD2-I is

66 a sliding clamp that encircles dsDNA at the stall site ${ }^{20,21}$; in parallel, the stalled fork may

67 undergo asymmetric fork reversal ${ }^{22}$. The motor proteins that mediate fork reversal during FA

68 pathway activation are unknown; FANCM is one candidate, since it can reverse model fork

69 structures in vitro and its motor activity is required for ICL resistance ${ }^{19,23,24}$. The combination of

70 fork reversal and FANCD2-I activation at the ICL promotes recruitment of the endonuclease

71 scaffold SLX4/FANCP and its associated endonuclease XPF (xeroderma pigmentosum group

72 F)/FANCQ in complex with ERCC1 (excision repair cross complementation group 1) ${ }^{25}$. XPF

73 makes incisions either side of the ICL on one sister chromatid, generating a two-ended double

74 strand break (DSB) opposite an 'unhooked' ICL on the unbroken sister chromatid. The single

75 stranded (ss)DNA gap opposite the unhooked ICL is filled by translesion synthesis (TLS) DNA

76 polymerases, and the DSB is repaired by conservative, two-ended HR. Indeed, several core HR

77 genes are also FA genes. These include the hereditary breast and ovarian cancer predisposition

78 genes $B R C A 1 / F A N C S$ and $B R C A 2 / F A N C D 1$, as well as the central mitotic recombinase

$79 R A D 51 / F A N C R$ and certain $R A D 51$ paralogs ${ }^{9}$. Despite these advances, it is unclear whether the

80 full FA mechanism is restricted to ICL repair, or whether FA-mediated incisions can induce HR

81 at other types of fork-stalling lesions, such as DNA-protein complexes ${ }^{26}$. In this regard, a

82 molecularly defined system that recapitulates the FA mechanism in vivo on a mammalian

83 chromosome has been lacking. 
85 To study HR triggered by replication fork stalling at a defined chromosomal locus of cycling mammalian cells, we previously adapted the Escherichia coli Tus/Ter replication fork barrier

87 (RFB) for use in mammalian cells ${ }^{27}$. We found that an array of six tandem $23 \mathrm{bp}$ Ter repeats,

88 when targeted to a specific genomic locus and bound by the Tus protein, triggers bidirectional

89 fork stalling and HR at the Tus/Ter RFB. The Tus/Ter RFB is a DNA-protein barrier lacking

90 covalent DNA-protein crosslinks or ICLs. Fork stalling events at Tus/Ter do not map to a single

91 defined nucleotide, but are distributed over the $\sim 200 \mathrm{bp} 6 \mathrm{xTer}$ array ${ }^{28}$. Thus, Tus/Ter-stalled

92 forks do not present a 'classical' ICL substrate for the FA pathway. It is therefore important to

93 determine the mechanisms underlying Tus/Ter-induced HR from first principles. Does it entail

94 formation of a DSB intermediate, analogous to the DSB intermediate generated by SLX4-XPF

95 during replication-coupled ICL repair by the FA pathway? Alternatively, does Tus/Ter-induced

96 HR resemble HR induced at the Schizosaccharomyces pombe RTS1 RFB, which does not

97 proceed via a DSB intermediate ${ }^{29}$ ?

99 The major conservative HR outcome at the Tus/Ter RFB, 'short tract' gene conversion (STGC),

100 is a product of two-ended HR, implicating bidirectional fork stalling in its initiation. Tus/Ter-

101 induced STGC is a non-crossover outcome mediated by the canonical BRCA-Rad51 HR

102 pathway ${ }^{27,28,30}$. These findings implicate the non-crossover 'synthesis-dependent strand

103 annealing' (SDSA) pathway as the underlying HR mechanism ${ }^{30,31}$. We found that FANCM

104 motor protein function and FANCM-mediated recruitment of the FA core complex are

105 specifically required for efficient Tus/Ter-induced STGC ${ }^{24}$. Indeed, FANCM and FA core genes

106 act epistatically in this function. Thus, Tus/Ter-induced STGC is mediated by early components

107 of the FA pathway and by canonical HR proteins. Whether Tus/Ter-induced STGC also entails 
an SLX4-mediated incision step, analogous to the unhooking step of FA pathway-mediated ICL repair, is currently unknown.

$111 S L X 4 / F A N C P$ encodes a scaffolding protein that binds multiple endonuclease complexes in a cell

112 cycle-dependent manner to cleave branched DNA structures ${ }^{32-38}$. In S phase cells, SLX4

113 interacts with the XPF-ERCC1 heterodimer and SLX1 (synthetic lethal of unknown function 1)

114 via the SLX4 MLR (MEI9- Interaction-Like Region) and SBD (SLX1-binding) domains,

115 respectively, as well as with MutS $\beta$ via the SLX4 N-terminus. The SLX4-XPF interaction is

116 required for cellular resistance to $\mathrm{ICLs}^{39}$. XPF-ERCC1 binds and cleaves the 3' arm of

117 replication fork-like substrates containing an ICL, but can also cleave dsDNA internal to the

118 branch junction, leading to ICL unhooking ${ }^{25,40-42}$. SLX4 binding enhances XPF nuclease activity

119 and increases its specificity for replication fork substrates. In mitosis, SLX4 additionally binds

120 nucleases MUS81 (methyl methanesulfonate and UV sensitive 81)-EME1 (essential meiotic

121 endonuclease 1) via the SAP (SAF-A/B, Acinus and PIAS) domain. These interactions enable

122 the SLX4 'trinuclease' complex to act on a variety of branched DNA substrates in mitosis,

123 cleaving stalled replication forks at sites of incomplete DNA synthesis and resolving post-

124 replicative Holliday junctions ${ }^{32-36}$. MUS81-EME1 preferentially cleaves 3' flap structures and

125 nicked Holliday junctions ${ }^{43-45}$. The preferred substrate for SLX1 is a 5 ' flap, which it cuts at the

126 ssDNA-dsDNA junction ${ }^{46}$. SLX4 binding enhances SLX1 substrate promiscuity, allowing

127 SLX1 to cleave splayed arms, 5' flaps, 3' flaps and Holliday junctions. SLX4 contains at its N-

128 terminus two conserved Ubiquitin Binding Domain 4 (UBZ) motifs that facilitate its localization

129 to laser-induced damage sites, implicating tethering of the UBZ motifs to as yet unidentified 
130 K63-linked poly-ubiquitinated substrates at sites of laser damage ${ }^{47,48}$. The role of the SLX4

131 tandem UBZ domain in SLX4 function at stalled mammalian replication forks is unknown.

133 Biallelic SLX4/FANCP mutations have been identified in several FA patients ${ }^{47,49}$. Two

134 independent FA kindreds were found to carry germ line SLX4 mutations that delete in-frame one 135 or both UBZ motifs. Another FA patient was found to carry a 1-bp deletion (c.286delA) in exon 1361 of $S L X 4$, resulting in an early frameshift, and a premature stop at codon 126

137 (p.Thr96Leu $f$ X30) in the second allele. Despite this disruption of the SLX4 open reading frame 138 (ORF), translation initiation at an alternative start site produced low levels of truncated protein in 139 cells from the affected individual ${ }^{49}$. Thus, FA-associated SLX4 mutations described to date are 140 hypomorphic alleles, encoding polypeptides with disrupted UBZ motifs. Possibly, more severe $141 S L X 4$ mutations are incompatible with human development. Indeed, mice carrying biallelic Slx4 142 germ line mutations are born in sub-mendelian ratios and reveal prominent growth defects ${ }^{50}$, 143 while biallelic deletion of SLX4 in chicken DT40 lymphoblastoid cells is cell lethal ${ }^{51}$.

144 Pathogenic mutations in $X P F / F A N C Q$ also cause FA ${ }^{52,53}$. Whether and how XPF contributes to 145 mammalian HR remains to be determined.

147 SLX4, together with MUS81, has been implicated in mitotic DNA synthesis (MiDAS) at 148 chromosomal 'fragile sites' — a form of aberrant replication restart that occurs when cells enter 149 mitosis with incompletely replicated chromosomes ${ }^{54,55}$. MiDAS is mediated by RAD52, SLX4, 150 MUS81-EME1, RTEL1 and POL $\delta$. Current models propose that SLX4-MUS81 initiates MiDAS 151 by breaking isolated stalled replication forks in mitotic cells, provoking break-induced 152 replication (BIR) at the resulting one-ended breaks. Aberrant replication restart is also observed 
153 in response to a Tus/Ter RFB, leading to 'long tract' gene conversion (LTGC) and, in BRCA1-

154 defective cells, the formation of non-homologous tandem duplications (TDs) ${ }^{28}$. Tus/Ter-induced

155 LTGC and TD formation are suppressed by FANCM and the Bloom's syndrome helicase BLM, 156 suggesting an underlying BIR-like migrating bubble mechanism ${ }^{24,30}$. The cell cycle phases in 157 which Tus/Ter-induced STGC, LTGC and TD formation occur, and thus their relationships to 158 MiDAS, are unknown.

160 It is also unknown whether SLX4 and its associated nucleases have roles in HR downstream of

161 DSB formation. In wild type cells, the majority of HR events triggered by a nuclease-induced

162 site-specific DSB resolve as STGC-a conservative HR outcome mediated by SDSA ${ }^{30}$. A

163 minority ( $\sim 5 \%)$ resolve as LTGC — an aberrant replicative 'gap repair' outcome of HR,

164 characterized by gene conversions of $>3 \mathrm{~kb}^{56-59}$. Work in yeast has emphasized similarities

165 between LTGC and BIR, and recent work in mammalian cells supports this connection ${ }^{60}$.

166 Although DSB-induced STGC and LTGC are both Rad51-dependent, mutation of certain core

167 HR genes disproportionately impairs STGC, resulting in a bias in favor of LTGC ${ }^{27,57,58}$. The

168 identification of genes that specifically support LTGC is important for understanding

169 mechanisms of genomic instability in human disease. In this regard, a number of different

170 experimental models implicate DNA polymerase $\delta$ in LTGC ${ }^{60-62}$. Recent studies additionally

171 identified the MCM8-MCM9-MCM8IP/HROB helicase complex and the PIF1 helicase as

172 mediators of mammalian DSB-induced LTGC ${ }^{60,63,64}$. It is unknown whether DSB-induced

173 LTGC is primarily S phase-restricted or, alternatively, a G2/M process potentially related to

174 MiDAS. SLX4 may be a useful tool with which to study this question. 
176 In this study, we use CRISPR/Cas9 genome editing to engineer precise, defined mutations in the

177 Slx4 gene of mouse embryonic stem (mES) cells that carry a reporter of stalled fork- and DSB-

178 induced HR. We systematically examine the role of individual nuclease-interacting domains of

179 SLX4 in stalled fork HR. Our results identify a critical role for the SLX4-XPF complex in

180 Tus/Ter-induced STGC. We define a requirement for the SLX4 UBZ motifs in the recruitment of

181 SLX4 to stalled forks, providing important insight into the mechanisms that connect clinically-

182 described $S L X 4 / F A N C P$ mutations to genomic instability. We also identify an unexpected role

183 for SLX4 in supporting LTGC in the repair of a replication-independent DSB.

\section{Results}

186 Slx4 regulates error-free HR at Tus/Ter-stalled replication forks.

187 To study the role of Slx4 in mammalian stalled fork repair, we used dual CRISPR/Cas9-mediated

188 incisions to introduce defined mutations into the endogenous $S l x 4$ gene of mouse embryonic

189 stem (ES) cells that contain a single copy of a 6xTer-HR reporter targeted to the Rosa26 locus on

190 chromosome VI ${ }^{27}$ (Fig. 1a). In this reporter, HR can be triggered by either Tus/Ter-induced fork

191 stalling or by a site-specific DSB induced by the rare-cutting homing endonuclease I-SceI. The

192 reporter enables simultaneous flow cytometric quantitation of error-free HR as "short tract" gene

193 conversion (STGC; $\mathrm{GFP}^{+} \mathrm{RFP}^{-}$) outcomes, and aberrant replicative HR responses as "long tract"

194 gene conversion (LTGC; $\mathrm{GFP}^{+} \mathrm{RFP}^{+}$) products. We engineered a $125 \mathrm{bp}$ frame-shift deletion in

195 exon $2\left(S l x 4^{\Delta 125}\right)$, thereby disrupting $S l x 4$ early in the ORF (Fig. 1b), and likely generating a

196 larger undefined deletion $\left(S l \times 4^{\Delta}\right)$ within the second allele ${ }^{24,65}$. We assayed two independent $S l \times 4$

197 mutant clones $\left(S l \times 4^{\Delta 125 / \Delta} \# 40,42\right)$ and two isogenic clones that received the same CRISPR/Cas9

198 treatment but retained wild type $\operatorname{Slx} 4\left(S l x 4^{+/+} \# 3,13\right)$ (Fig. 1b). Both $S l x 4^{\Delta 125 / \Delta}$ clones expressed 
199 normal levels of Slx 4 mRNA (Supplementary Fig. 1), suggesting that $S l x 4^{\Delta 125}$ may not be

200 functionally a true null allele. To determine the sensitivity of $S l x 4^{\Delta 125 / \Delta}$ clones to the ICL-

201 inducing agent mitomycin C (MMC), we mixed $\sim 80 \%$ uncolored (GFP-) Slx $4^{\Delta 125 / \Delta}$ mutants (or

202 isogenic $\mathrm{Sl} 4^{+/+} \mathrm{GFP}^{-}$controls) with $\sim 20 \% \mathrm{GFP}^{+}$wild type cells and exposed them to titrated

203 concentrations of MMC for 48 hours (Fig. 1c). We observed a significant enrichment of GFP

204 cells in $S l x 4^{\Delta 125 / \Delta}$ mixed populations, implying loss of viability of $S l x 4^{\Delta 125 / \Delta}$ cells in comparison

205 to $\mathrm{GFP}^{+}$wild type cells (Fig. 1c). Thus, as expected, Slx $4^{\Delta 125 / \Delta}$ mutants are sensitive to MMC.

207 To measure stalled fork HR and DSB-induced HR, we transfected Ter-HR reporter cells, in

208 parallel, with Tus or I-SceI expression vectors and assayed repair products 72 hours post-

209 transfection (see methods). Each $S l x 4^{\Delta 125 / \Delta}$ clone revealed a $\sim 4$-fold reduction in Tus/Ter induced

210 STGC compared to the two isogenic wild type clones, whereas Tus/Ter-induced LTGC was

211 unaffected by Slx4 disruption (Fig. 1d, 1e and 1f). Hence, HR at Tus/Ter was skewed in favor of

212 LTGC (Supplementary Fig. 1). In contrast, I-SceI-induced STGC was unaltered in Slx $4^{\Delta 125 / \Delta}$

213 cells, whereas I-SceI-mediated LTGC was reduced (Fig. 1d, 1g and 1h). Hence, in DSB-induced

214 HR, the ratio of LTGC:Total HR was reduced 2-fold in Slx $4^{\Delta 125 / \Delta}$ cells (Supplementary Fig. 1).

215 These results identify distinct roles for mammalian Slx4 in stalled fork HR and in conventional

216 DSB-induced HR. Specifically, the data suggest that Slx4 mediates error-free HR (i.e., STGC) at

217 stalled replication forks but not at a replication-independent DSB. Conversely, Slx 4 specifically

218 mediates DSB-induced LTGC, but is dispensable for Tus/Ter-induced LTGC.

219

220 Slx4 hemizygous cells phenocopy wild type cells 
221 To facilitate analysis of individual SLX4 domains in stalled fork repair, we used dual

222 CRISPR/Cas9 incisions at exon 2 and exon 15 of Slx4 to delete one copy of the entire $19.3 \mathrm{~kb}$

223 gene (Supplementary Fig. 1). We retrieved 202/262 Slx $4^{+/+}$clones from this experiment, 60/262

$224 S l x 4^{+/-}$clones but no Slx $x 4^{-/-}$clones. The expected $S l x 4^{-/-}$frequency was $(60 / 262)^{2}=\sim 13 / 262$.

225 This result therefore suggests that $S l x 4^{-/-}$is cell lethal. We used direct sequencing of breakpoint

226 PCR products to identify the deletion breakpoint in $S l x 4^{+/-}$cells and to confirm that the retained

227 allele is wild type at the sgRNA target sites (Supplementary Fig. 1). Slx4 expression in the

228 Sl $x 4^{+/}$clone was at wild type levels, suggesting that transcription is upregulated in

229 compensation for hemizygosity (Supplementary Fig. 1). Importantly, Slx $4^{+/}$cells revealed wild

230 type HR repair frequencies (Supplementary Fig. 1). Thus, $S l \times 4^{+/}$mES cells are phenotypically

231 wild type for stalled fork- and DSB-induced HR.

232

\section{The SLX4 UBZ domain recruits SLX4 to stalled forks to mediate error-free HR}

234 To study the role of the SLX4 UBZ domain in Tus/Ter-mediated HR, we used dual

235 CRISPR/Cas9-mediated incisions to delete in-frame the UBZ1- and UBZ2-coding regions in

$236 S l x 4^{+/}$Ter-HR reporter cells (Fig. 2a, Supplementary Fig. 2). UBZ-encoding mRNA sequences

237 were undetectable in $S l x 4^{\mathrm{AUBZ} /}$ cells, whereas sequences downstream (encoding MLR, SAP and

238 SBD domains) were expressed at normal levels (Supplementary Fig. 2). We performed

239 competitive growth assays using three independent $S l x 4^{\Delta \mathrm{UBZ} /-}$ clones $(\# 1,2,5)$ and three

240 independent sgRNA/Cas9 treated, yet unaltered, $S l x 4^{+/}$clones $(\# 7,25,44)$, in the presence of

241 MMC or Olaparib. All Sl $x 4^{\Delta \mathrm{UBZ} /-}$ clones were hypersensitive to MMC (Supplementary Fig. 2)

242 and were resistant to Olaparib (Supplementary Fig. 2). A characteristic cellular feature of FA is

243 increased late S/G2 phase enrichment following exposure to MMC ${ }^{9}$. Untreated Slx $4^{\Delta \mathrm{UBZ} /-}$ cells 
244 revealed cell cycle distribution patterns similar to $S l x 4^{+/}$cells (Fig. 2b). However, in response to

245 titrated doses of MMC, Slx $4^{\Delta \mathrm{UBZ} /-}$ cells accumulated in G2/M more readily than Slx $4^{+/-}$controls,

246 and revealed a corresponding reduction in the S-phase fraction (Fig. 2b).

248 Next, we analyzed six independent $S l x 4^{\Delta \mathrm{UBZ} /-}$ clones and six similarly Cas9/sgRNA-exposed 249 independent $S l x 4^{+/}$clones in stalled fork repair and DSB repair. Strikingly, Tus/Ter-induced 250 STGC was reduced $\sim 6$-fold in $S l x 4^{\Delta \mathrm{UBZ} /-}$ clones in comparison to $S l x 4^{+/-}$controls (Fig. 2c). In 251 contrast, the frequency of I-SceI induced STGC was only modestly reduced (Fig. 2d). Transient 252 expression of wild type human SLX4 restored Tus/Ter-induced STGC to wild type levels in 253 Sl $x 4^{\triangle \mathrm{UBZ} /-}$ clones (\#2 and \#5), but had no impact on Slx $4^{+/-}$controls (\#25 and \#44) (Fig. 2e and

254 Supplementary Fig. 2). A UBZ 4C $>$ A mutant that specifically disrupts UBZ domain function ${ }^{39}$

255 failed to complement the STGC defect of Slx $4^{\Delta \mathrm{UBZ} /-}$ cells, despite equivalent levels of

256 expression. In the same experiment, expression of wild type $S L X 4$ or $4 \mathrm{C}>\mathrm{A}$ had no statistically

257 significant impact on I-SceI-induced STGC in either $S l x 4^{\Delta \mathrm{UBZ} /-}$ or $S l x 4^{+/-}$clones (Fig. 2f). The 258 ability of wild type SLX4 but not the 4C $>$ A mutant to complement the defect in Tus/Ter-induced 259 STGC in Slx $4^{\Delta \mathrm{UBZ} /-}$ cells indicates a specific requirement for the SLX4 UBZ domain in error-free 260 HR (i.e., STGC) at stalled forks. In contrast, the SLX4 UBZ domain has no clear role in DSB261 induced STGC. Thus, $S l x 4^{\Delta \mathrm{UBZ}}$ is a separation-of-function allele that distinguishes control of 262 STGC at stalled forks from STGC at replication-independent DSBs.

264 The same experiments revealed different effects on LTGC. Tus/Ter-induced LTGC was only 265 minimally reduced in the six $S l x 4^{\Delta \mathrm{UBZ} /-}$ clones; hence, the ratio of LTGC:Total HR was elevated, 266 reflecting the severe defect in STGC (Supplementary Fig. 2). Tus/Ter-induced LTGC was 
267 unaffected by expression of either wild type SLX4 or the 4C>A mutant (Supplementary Fig. 2).

268 Thus, there is no clear evidence of a role for the SLX4 UBZ domain in Tus/Ter-induced LTGC.

269 In contrast, I-SceI-induced LTGC was reduced $>2$-fold in the six $S l x 4^{\Delta \mathrm{UBZ} /-}$ clones in comparison

270 to Slx $4^{+/}$controls; as a result, the ratio of LTGC:Total HR was reduced $\sim 2$-fold, reflecting the

271 specific reduction in I-SceI-induced LTGC (Supplementary Fig. 2). The LTGC defect was

272 complemented by wild type SLX4 but not by the 4C>A mutant (Supplementary Fig. 2). Thus,

$273 S l \times 4^{\Delta \mathrm{UBZ}}$ is a separation-of-function allele that distinguishes control of LTGC at replication-

274 independent DSBs from LTGC at stalled forks.

275

276 To facilitate detection of the endogenous Slx4 gene product in mouse ES cells, we targeted the

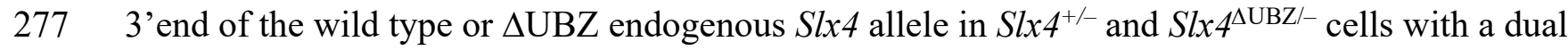

278 minimal auxin-inducible and SMASh degron containing an 8x HA epitope tag positioned N-

279 terminal of the SMASh degron cleavage site ${ }^{24,66-68}$, to generate $S l x 4^{\text {deg } /-}$ and $S l x 4^{\text {AUBZdeg } /-}$ clones

280 (Fig. 2g). Slx $4^{\text {deg } /-}$ and $S l x 4^{\Delta \mathrm{UBZdeg} /-}$ clones expressed comparable levels of Slx4 mRNA (Fig. 2h);

281 HA-tagged gene products were detected in the chromatin fraction but not in whole cell extracts.

282 Unexpectedly, the HA-tagged $S l \times 4^{\Delta \mathrm{UBZdeg}}$ gene product was detected at higher levels in the

283 chromatin fraction than the wild type protein (Fig. 2i). Activation of the dual degron triggered

284 partial degradation of endogenous SLX4, confirming the specificity of the anti-HA western blot

285 signal. We used these tools, in combination with chromatin immunoprecipitation (ChIP), to

286 study SLX4 recruitment to Tus/Ter-stalled forks. We noted robust recruitment of wild type

287 SLX4-deg to the Tus/Ter RFB (Fig. 2j). Strikingly, despite its greater abundance in chromatin

288 fractions, SLX4- $\Delta$ UBZdeg was detected at much lower levels at Tus/Ter by ChIP. Thus, the 
SLX4 UBZ domain mediates Tus/Ter-induced STGC by promoting SLX4 accumulation at sites

290 of fork stalling.

292 Interaction of SLX4 with MUS81 or SLX1 is dispensable for Tus/Ter-induced and DSB293 induced HR

294 Interactions of SLX4 with the endonucleases MUS81 and SLX1 in mitosis facilitate cleavage of 295 branched DNA structures, including stalled replication forks and Holliday junctions (HJs) ${ }^{32}$. To 296 study the SLX4-MUS81 interaction in Tus/Ter-induced repair, we used Cas9-dual sgRNA 297 incisions to generate in-frame deletions of Slx4 exons 11-13, encoding the SAP domain, in $298 S l x 4^{+/-}$Ter-HR reporter cells (Fig. 3a and Supplementary Fig. 3). The mRNA sequence 299 encoding the SAP domain was undetectable in $S l x 4^{\Delta \mathrm{SAP} /-}$ cells, whereas expression of other $S l x 4$ 300 elements was at wild type levels (Supplementary Fig. 3). Consistent with a previous study ${ }^{39}$, 301 we found that three independent $\operatorname{Sl} x 4^{\Delta \mathrm{SAP} /-}$ clones (\#21, \#28, \#51) are modestly sensitive to 302 MMC and Olaparib (Fig. 3b and 3c) as compared to three $S l x 4^{+/-}$clones $(\# 5, \# 8, \# 17)$ that had 303 been similarly treated with Cas9/sgRNA. We analyzed stalled fork and DSB repair functions in 304 five independent $S l x 4^{\Delta \mathrm{SAP} /-}$ clones and five isogenic Sl $x 4^{+/-}$clones. Tus/Ter induced and I-SceI305 induced STGC and LTGC were indistinguishable between $S l x 4^{\Delta \mathrm{SAP} /-}$ and $S l x 4^{+/-}$clones (Fig. 3d, 306 3e and Supplementary Fig. 3). Thus, the SLX4 SAP domain and, hence, the interaction of 307 SLX4 with MUS81, is dispensable for these repair functions.

309 To study the SLX4-SLX1 interaction in Tus/Ter induced repair, we used Cas9-dual sgRNA 310 incisions to generate in-frame deletions of Slx4 exons 13-15, encoding the SBD domain, in $311 S l x 4^{+-}$Ter-HR reporter cells (Fig. 3f and Supplementary Fig. 3). The mRNA sequence 
312 encoding the SBD domain was almost undetectable in the $S l x 4^{\Delta \mathrm{SBD} /-}$ cells, whereas expression of

313 other elements of Slx4 was at normal levels (Supplementary Fig. 3). Competitive growth assays

314 revealed that $S l x 4^{\Delta \mathrm{SBD} /-}$ mutants (\#15, \#29, \#31) are not sensitive to MMC or Olaparib (Fig. $3 \mathrm{~g}$

315 and $\mathbf{3 h}$ ), as compared to $S l x 4^{+/-}$clones $(\# 2, \# 16, \# 22)$ that had been similarly treated with

316 Cas9/sgRNA. We analyzed stalled fork and DSB repair functions in six independent $S l x 4^{\Delta \mathrm{SBD} /-}$

317 clones and six isogenic $S l x 4^{+/-}$clones. No alterations in Tus/Ter-induced or I-SceI-induced

318 STGC and LTGC were detected (Fig. 3i, 3j, and Supplementary Fig. 3). Thus, the SLX4 SBD

319 domain and, hence, the interaction of SLX4 with SLX1 is dispensable for these repair functions.

\section{The SLX4-XPF interaction is required for STGC at stalled forks}

322 To study the SLX4-XPF interaction in Tus/Ter stalled fork repair, we used dual sgRNAs to 323 generate in-frame deletion of the MLR-encoding exons 5 and 6 in $S l x 4^{+/-}$Ter-HR reporter cells

324 (Fig. 4a, Supplementary Fig. 4). Slx 4 mRNA transcripts in $S l x 4^{\Delta \mathrm{MLR} /-}$ clones lacked the deleted 325 region but were otherwise detected at normal levels (Supplementary Fig. 4). Slx $4^{\Delta \mathrm{MLR} /-}$ clones 326 were slow-growing compared to isogenic $S l x 4^{+/-}$controls that had been similarly exposed to

327 Cas9/sgRNA but had retained wild type Slx4. In untreated cells, the proportion of cells in late $328 \mathrm{~S} / \mathrm{G} 2 / \mathrm{M}$ was elevated in $S l x 4^{\mathrm{MLLR} /-}$ cells $(24 \%)$ in comparison to $\operatorname{Sl} 4^{+/-}$cells (12\%; Fig. 4b). The 329 S-phase fraction was correspondingly reduced. Similarly, following exposure of cells to titrated 330 doses of MMC, Slx $4^{\Delta \mathrm{MLR} /-}$ cells revealed more dramatic late S/G2/M arrest and S phase depletion 331 than Slx $4^{+/-}$controls (Fig. 4b). Competitive growth assays confirmed that Slx $4^{\Delta \mathrm{MLR} /-}$ cells (\#5, $332 \# 20, \# 31)$ are hypersensitive to MMC but not to Olaparib in comparison to Slx $4^{+/-}$controls (\#13, $333 \# 17, \# 28$ ) (Supplementary Fig. 4). Slow growth and the increased late S/G2/M arrest is 334 consistent with the idea that $S l x 4^{\Delta \mathrm{MLR} /-}$ cells experience high levels of spontaneous replication 
335 stress. To explore this effect further, we analyzed metaphase spreads from $S l x 4^{+/-}, S l x 4^{\Delta U B Z /-}$ and

$336 S l x 4^{\triangle M L R /-}$ cultures that were either untreated or exposed to $20 \mathrm{ng} / \mathrm{ml}$ of MMC for 12 hours. Both

$337 S l \times 4^{\triangle U B Z /-}$ and $S l x 4^{\triangle M L R /-}$ cells revealed elevated levels of breaks and radial chromosomes in

338 comparison to $S l x 4^{+/-}$controls, but $S l x 4^{\triangle M L R /-}$ cells revealed a more severe defect than $S l x 4^{\Delta U B Z /-}$

339 cells (Fig. 4c, $4 \mathbf{d}$ and 4e). Thus, both $S l x 4^{\Delta U B Z /-}$ and $S l x 4^{\Delta M L R /-}$ cells display a classical Fanconi

340 anemia chromosome breakage phenotype. In a second experiment, we additionally analyzed

$341 S l \times 4^{\triangle S A P /-}$ and $S l x 4^{\triangle S B D /-}$ cells (Supplementary Fig. 4). Slx $4^{\triangle S A P /-}$ cells were indistinguishable

342 from $S l x 4^{+/-}$cells. Slx $4^{\Delta S B D /-}$ cells revealed minimally elevated frequencies of breaks and radials

343 compared to Slx $4^{+/-}$cells.

345 We analyzed stalled fork- and DSB-induced HR in six independent $S l x 4^{\Delta \mathrm{MLR} /-}$ clones and six 346 independent $S l x 4^{+/-}$isogenic clones. Tus/Ter-induced STGC was almost undetectable in 347 Sl $x 4^{\Delta \mathrm{MLR} /-}$ cells, whereas LTGC was detected at wild type levels (Fig. 5a and Supplementary

348 Fig. 5). We noted reduced I-SceI induced STGC in Slx $4^{\Delta \mathrm{MLR} /-}$ clones, while I-SceI induced

349 LTGC was marginally reduced (Fig. 5b and Supplementary Fig. 5). These findings suggest that

350 the SLX4 MLR domain and, hence the interaction of SLX4 with XPF, is required for Tus/Ter-

351 induced STGC and for efficient DSB-induced STGC. A caveat of attempts to quantify repair

352 directly in Slx $4^{\mathrm{MLR} /-}$ cells is the possibility that the high levels of replication stress in these cells

353 might artifactually alter the measured HR frequencies ${ }^{69}$. To address this concern, we performed

354 complementation experiments in Slx $4^{\Delta \mathrm{UBZ} /-}$ Ter-HR reporter cells, which exhibit no proliferation

355 defect. In addition to testing full-length $\mathrm{h} S L X 4$ expression vectors, we generated 'mini-SLX4'

356 constructs that encode hSLX4 polypeptides that are truncated C-terminal to the BTB domain and

357 therefore lack the MUS81-binding SAP or SLX1-binding SBD domains ${ }^{40}$. These mini-SLX4 
constructs included wild type, $4 \mathrm{C}>\mathrm{A}$ and $\triangle \mathrm{MLR}$ variants (Fig. 5c). Following transient transfection, hSLX4 was expressed equivalently from each construct (Fig. 5d). Consistent with

360 our above-noted findings that the SLX4 SAP and SBD domains are dispensable for Tus/Ter-

361 induced STGC, expression of mini-SLX4 complemented the defect in Tus/Ter-induced STGC in

$362 S l x 4^{\Delta \mathrm{UBZ} /-}$ cells as efficiently as wild type $S L X 4$ (Fig. 5f). Importantly, both full length

363 SLX4AMLR and mini-SLX4AMLR failed to complement the defect in Tus/Ter-induced STGC in $364 S l \times 4^{\Delta \mathrm{UBZ} /-}$ cells, behaving similarly to $S L X 44 \mathrm{C}>\mathrm{A}$ vectors. No significant effects of any of these 365 constructs were observed on Tus/Ter-induced STGC in Slx $4^{+/-}$cells, and I-SceI-induced STGC 366 was unaffected by any $S L X 4$ construct in either cell type (Fig. 5f). These results show that intact 367 SLX4 UBZ and MLR domains are independently required for Tus/Ter-induced STGC. The 368 absence of interallelic complementation between the endogenous $S l x 4^{\Delta \mathrm{UBZ}}$ and exogenous $369 S L X 4 \triangle \mathrm{MLR}$ alleles suggests that the UBZ and MLR domains must each be intact on a single 370 SLX4 molecule in order for SLX4 to mediate Tus/Ter-induced STGC.

372 In the same set of experiments, Tus/Ter-induced LTGC was unaffected by any of the above373 noted $\mathrm{h} S L X 4$ constructs in either $S l x 4^{\Delta \mathrm{UBZ} /-}$ or $S l x 4^{+/-}$cells (Supplementary Fig. 5). In contrast, 374 I-SceI-induced LTGC, which is specifically impaired in Slx $4^{\Delta \mathrm{UBZ} /-}$ cells, was complemented by 375 expression of full-length wild type $S L X 4$ (consistent with our previous experiments), but not by 376 the $\triangle$ MLR mutant (Supplementary Fig. 5). Similarly, wild type mini-SLX4 complemented the 377 defect in I-SceI-induced LTGC, whereas mini-SLX4 mutants 4C $>$ A and $\triangle$ MLR failed to do so. 378 These results suggest that I-SceI-induced LTGC requires both the UBZ and MLR domains of 379 SLX4, but is independent of domains distal to the BTB dimerization domain. 
382 The above findings implicate the SLX4-XPF interaction in Tus/Ter-induced STGC. We therefore 383 used CRISPR/Cas9 with dual sgRNA targeting to delete the one entire $\sim 42.3 \mathrm{~kb} X p f$ allele in $384 T e r$-HR reporter cells (Supplementary Fig. 6). $X p f$ expression was reduced by $\sim 50 \%$ in $X p f^{+/-}$ 385 cells compared to an isogenic $X p f^{4 /+}$ clone, implying an absence of transcriptional compensation 386 for hemizygosity (Supplementary Fig. 6). Nonetheless, $X p f^{+/-} T e r$-HR reporter cells revealed 387 wild type repair frequencies (Supplementary Fig. 6). To determine whether the XPF nuclease 388 domain contributes to stalled fork HR, we used Cas9 with dual sgRNAs to delete in-frame the 389 nuclease domain-encoding region of $X p f$ in $X p f^{4 /-}$ cells (Fig. 6a and $6 \mathbf{b}$ ). We obtained one $390 X p f^{\text {Nuc/ }}$ clone containing an in-frame deletion (\#1) and three that contained a frameshift (\#2, \#11, $391 \# 13)$. Analysis of Xpf expression in each mutant clone revealed the absence of nuclease domain392 encoding sequences but normal levels of expression of regions encoding the upstream helicase 393 domain and the downstream $\mathrm{HhH} 2$ domain (Supplementary Fig. 6). This finding suggests that 394 there was no nonsense-mediated decay of frame-shifted transcripts. We were unable to detect the 395 endogenous XPF protein in mES cells. Western blot analysis of the HhH2-binding XPF 396 heterodimeric partner ERCC1 in whole cell extracts revealed variably reduced abundance of

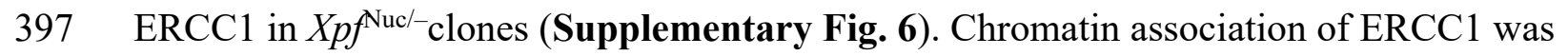

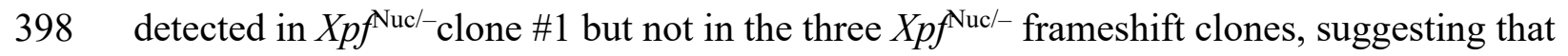
399 interaction of XPF with ERCC1 is required for chromatin association of ERCC1

400 (Supplementary Fig. 6). All four $X p \mathrm{f}^{\mathrm{Nuc} /}$ clones displayed a proliferative defect similar to that 401 of Slx $4^{\triangle \mathrm{MLR} /-}$ cells, associated with in an increase in the G2/M fraction and a reduction in the S402 phase fraction in unperturbed cells (Supplementary Fig. 6). Similarly, following exposure of 403 cells to titrated doses of MMC, $X p f^{\mathrm{Nuc} /}$ cells revealed more dramatic G2/M arrest and S phase 
404 depletion than $X p f^{+/-}$controls. Competitive growth assays corroborated these findings, revealing 405 that $X p f^{\text {Nuc/- }}$ cells are hypersensitive to MMC, but not to Olaparib, in comparison to $X p f^{+/-}$

406 controls (Fig. 6c and 6d). We prepared metaphase spreads from $X p f^{\mathrm{Nuc} /}$ or $X p f^{+/-}$cells that were 407 either untreated or exposed to low dose MMC. Strikingly, $X p f^{\text {Nuc/- }}$ cells revealed dramatically 408 elevated frequencies of MMC-induced chromatid breaks and radials, consistent with a classical

409 FA phenotype (Fig. 6e, $6 \mathbf{6}$ and $\mathbf{6 g}$ ). These findings underscore the importance of $X P F / F A N C Q$ as 410 a bona fide FA gene.

412 We analyzed stalled fork and DSB repair functions in each of the four $X p f^{\mathrm{Nuc} /-} \mathrm{Ter}$-HR reporter 413 clones and four isogenic $X p f^{+/-}$clones. Similar to $S l x 4^{\mathrm{MLLR} /-}$ cells, $X p f^{\mathrm{Nuc} /}$ cells revealed near414 complete loss of Tus/Ter-induced STGC (Fig. 6h) but no detectable alteration in Tus/Ter 415 induced LTGC (Supplementary Fig. 6). We also noted a significant reduction in I-Scel induced 416 STGC (Fig. 6i) and a modest reduction in I-SceI induced LTGC (Supplementary Fig. 6). Thus, $417 X p f^{\mathrm{Nuc} /}$ cells phenocopy $S l \times 4^{\Delta \mathrm{MLR} /}$ cells in stalled fork repair. The slow growth phenotype of

$418 X p f^{\text {Nuc/ }}$ cells suggests that these repair frequencies should be interpreted with caution. However, 419 it is notable that all the experimental systems described herein point to SLX4-XPF as a critical 420 mediator Tus/Ter-induced STGC. Further, the Slx4 and Xpf mutants that revealed the most 421 severe FA phenotype, in classical assays of MMC sensitivity and MMC-induced chromatid-type 422 aberrations, also exhibited the most profound impairment of Tus/Ter-induced STGC.

\section{Discussion}

425 We show here that the structure-specific endonuclease scaffold SLX4 regulates distinct HR 426 pathways at stalled replication forks and at replication-independent DSBs. In response to fork 
427 stalling, two-ended, conservative HR (i.e., STGC) is dependent on Slx4, while DSB-induced

428 STGC is either unaffected by or less sensitive to Slx4 mutation. Conversely, DSB-induced

429 LTGC, a replicative HR outcome possibly related to BIR, is Slx4-dependent, while LTGC at

430 stalled forks is unaffected by Slx4 mutation.

432 Tus/Ter-induced STGC is a product of the Fanconi anemia pathway

433 Our previous work showed that Tus/Ter-induced STGC is dependent on the early steps of the FA

434 pathway of ICL repair. Similar to FA-mediated ICL repair, Tus/Ter-induced STGC is a product

435 of two-ended HR, implicating bidirectional fork stalling as an initiating event ${ }^{27}$. The stalled fork

436 scaffold and motor protein FANCM regulates Tus/Ter-induced STGC by recruiting the FA core

437 complex to stalled forks through direct interaction with FANCF ${ }^{24,70}$. A Fancm mutation that

438 disrupts FANCF binding is specifically defective for Tus/Ter-induced STGC, and this mutation

439 is epistatic with loss of FA core complex proteins FANCA and FANCL. Experiments reported

440 here suggest that Tus/Ter-induced STGC is almost completely dependent on a later step of the

441 FA pathway, involving SLX4 and the XPF nuclease. We propose that SLX4-XPF controls

442 Tus/Ter-induced STGC by introducing one or more incisions at the site of bidirectional fork

443 arrest (Fig. 7). The resulting two-ended DSB intermediate is then repaired by canonical

444 HR/SDSA. In other words, the role of SLX4-XPF in Tus/Ter-induced STGC recapitulates its

445 role in the 'unhooking' step of ICL repair ${ }^{8,25,42}$. However, in the absence of an ICL at the stall

446 site, only one XPF-mediated incision of a stalled leading parental strand would be required to

447 generate a two-ended DSB at Tus/Ter (Fig. 7). Thus, the minimal requirements for two-ended

448 DSB formation at Tus/Ter may be less stringent than at an ICL. The proposed model would

449 explain why SLX4 plays a major role in Tus/Ter-induced STGC but a much less significant role 
450 in I-SceI-induced STGC. In the former case, if SLX4-XPF coordination at the stalled fork is

451 impaired, production of a DSB at the stall site is suppressed, resulting in failure of FA-mediated

452 HR and low levels of Tus/Ter-induced STGC. In the latter case, the DSB is induced by the I-SceI

453 endonuclease, bypassing any requirement for SLX4-XPF in DSB formation.

455 This model raises additional important questions regarding mechanism. In FA-mediated ICL 456 repair, close proximity of the two opposing replisomes is required to support TRAIP-mediated

457 ubiquitination of opposing CMG complex in trans, leading to FA pathway activation ${ }^{16}$. It might 458 appear that the $\sim 200$ bp Tus/Ter RFB would not allow the two opposing replisomes to approach

459 sufficiently closely to one another to allow TRAIP-mediated CMG ubiquitination in trans.

460 However, the Tus/Ter RFB is not an absolute barrier to the replicative helicase. Our previous 461 study using high throughput genomewide translocation sequencing showed that fork stalling 462 events are distributed throughout the $6 x$ Ter array ${ }^{28}$. Thus, the Tus/Ter RFB might be compatible 463 with productive TRAIP activation. These considerations suggest that the full FA mechanism 464 might indeed be activated at Tus/Ter-stalled forks, consistent with the findings presented here. In 465 light of these findings, it will be important in future experiments to determine whether Tus/Ter466 induced STGC requires TRAIP-mediated CMG ubiquitination, monoubiquitination of FANCD2467 FANCI and/or a fork reversal step ${ }^{22}$.

469 Our findings extend the scope of the FA pathway beyond ICL repair and answer in the 470 affirmative the longstanding question of whether the FA pathway can process a DNA-protein 471 replication barrier for $\mathrm{HR}^{26}$. Notably, aldehydes, a class of chemicals to which FA mutant cells 472 are hypersensitive, can form both ICLs and DNA-protein crosslinks (DPCs) ${ }^{6,11-14}$. Conceivably, 
473 some aldehyde-induced DPCs encountered during replication might escape proteolytic

474 mechanisms of DPC bypass and be processed by the FA pathway for HR ${ }^{26,71-74}$. This hypothesis

475 predicts that the sensitivity of FA mutant cells to aldehydes is, in part, a reflection of the inability

476 of these cells to process aldehyde-induced DPCs for HR.

478 Our genetic analysis of SLX4 shows that the UBZ and MLR domains are each independently 479 required for Tus/Ter-induced STGC. The absence of inter-allelic complementation between the 480 endogenous $S l x 4^{\triangle \mathrm{UBZ}}$ and exogenous $S L X 4^{\triangle \mathrm{MLR}}$ alleles (Fig. 5e) suggests that each of the UBZ 481 and MLR domains must be intact on a single SLX4 molecule for this function to be supported. In 482 contrast, the SAP and SBD domains are fully dispensable for Tus/Ter-induced STGC. This result 483 shows that Tus/Ter-induced STGC does not require assembly of the mitosis-associated SLX4 484 trinuclease complex. Rather, Tus/Ter-induced STGC appears to be coupled to S phase, when 485 SLX4-XPF interactions dominate.

487 We show that a key function of the SLX4 UBZ domain is to support efficient retention of SLX4 488 at the stall site. This finding is important in light of recent structural studies, which showed that 489 activated (monoubiquitinated) FANCD2-FANCI forms a sliding clamp on dsDNA ${ }^{20,75}$.

490 Importantly, the ubiquitin marks associated with FANCD2-FANCI are buried within the barrel 491 of the heterodimer and are unavailable for binding to SLX4 ${ }^{21}$. Our findings underscore the 492 importance of ubiquitin binding by SLX4 in stalled fork HR; the identity of the ubiquitinated 493 substrate that anchors SLX4 at replication stall sites remains to be discovered. The Slx $4^{\mathrm{ABZ} /-}$ 494 phenotype also sheds light on clinical cases of Fanconi anemia associated with SXL4/FANCP 495 mutation. As noted earlier, FA-associated SLX4 mutations are hypomorphs, implicating 
496 inactivation of UBZ domain function as a common defect in FANCP patients ${ }^{47,49}$. Our data 497 suggest that a key defect in FANCP mutant FA patient cells is ineffective retention of SLX4 at 498 stalled forks. Unlike the true $S L X 4$ null, which our data suggests is cell lethal when homozygous, 499 germ line homozygous SLX4 $\triangle \mathrm{UBZ}$ mutations may be compatible with human development 500 because the SLX4 retention defect is quantitative rather than absolute.

\section{Role of SLX4 in DSB-induced HR}

503 Our work unexpectedly identified SLX4 as a mediator of mammalian DSB-induced LTGC. This 504 finding might appear to connect DSB-induced LTGC to MiDAS, which is also mediated by 505 SLX4. However, unlike the role of SLX4 in MiDAS, where SLX4-MUS81 interaction is thought 506 to initiate the process by cleaving solitary arrested forks, we find that interaction of SLX4 with 507 either MUS81 or SLX1 is dispensable for DSB-induced LTGC, i.e., there is no indication of a 508 role for the mitotic SLX4 trinuclease complex. Instead, DSB-induced LTGC requires the SLX4 509 UBZ and MLR domains, implicating ubiquitin chain binding and the SLX4-XPF interaction in a 510 mechanism that operates subsequent to DSB induction. We show here that this Slx4-dependent 511 step, presumably involving XPF-mediated incisions, differentiates DSB-induced LTGC from

512 DSB-induced STGC. The non-involvement of SLX4 in Tus/Ter-induced LTGC further

513 underscores the idea that LTGC at stalled forks, which is Rad51-independent ${ }^{27}$, is the product of 514 a mechanism distinct from that of DSB-induced LTGC.

516 How SLX4 might promote DSB-induced LTGC is a matter of speculation. Both STGC and 517 LTGC are initiated by Rad51-mediated strand invasion by one end of the DSB, forming a D-loop 518 as a precursor to repair by SDSA ${ }^{30,31}$. Both pathways will require a 3' flap endonuclease to 
519 cleave non-homologous sequences from the invading strand. Perhaps the defects in I-SceI-

520 induced STGC we observed in $S l x 4^{\Delta \mathrm{MLR} /-}$ and $X p f^{\mathrm{Nuc} /}$ cells, but not in $S l x 4^{\Delta \mathrm{UBZ} /-}$ cells, reflect a

521 ubiquitin-binding-independent role for SLX4-XPF in this 3' flap-processing activity. DSB-

522 induced LTGC might be specifically favored if synapsis or processing of the two ends of the

523 DSB were impaired, or if normal maturation of the D-loop were disturbed ${ }^{57}$. Efficient branch

524 migration of the lagging edge of the D loop might normally promote nascent strand

525 displacement, thereby supporting timely HR termination and STGC. Conceivably, SLX4-XPF-

526 mediated incision of the trailing edge of the D-loop might disable the branch migration

527 mechanism that normally promotes STGC. This disruption of D-loop processing might stabilize

528 the nascent strand and thereby promote LTGC. We assume that such a role of SLX4-XPF in

529 DSB-induced LTGC would be opportunistic and pathological, favoring chromosome

530 rearrangements driven by LTGC and, potentially, BIR.

\section{Acknowledgements}

533 We thank Drs. Johannes Walter and Ketan J. Patel for helpful discussions. This work was

534 supported by grants R01GM134425 (to R.S.) and R01CA204127 (to A.S.), and by AACR

535 fellowship 19-40-12-PAND (to A.P.). A.S. is a Howard Hughes Faculty Scholar.

\section{Author Contributions}

538 R.E., A.P., F.P.L., N.A.W., and E.E.D. conducted the experiments; R.E., A.S. and R.S. designed 539 the experiments. R.E. and R.S wrote the manuscript.

\section{Declaration of Interests}


542 The authors declare no competing interests.

\section{$544 \quad$ Figure Legends}

Fig. 1. Slx4 regulates error-free HR at Tus/Ter stalled forks. a. Schematic of 6xTer-HR

547 reporter and repair products of Tus/Ter induced fork stalling. Light blue boxes: mutant GFP

548 alleles. Green box: wild type GFP. Open circles A and B: 5' and 3' artificial RFP exons,

549 respectively. 5' Tr-GFP: 5'-truncated GFP. Red triangle: 6xTer array. Black line: I-SceI

550 restriction site. STGC/LTGC, short/long tract gene conversion outcomes. LTGC generates wild-

551 type RFP through RNA splicing (red filled circles). b. Strategy for generation of Slx $4^{\Delta 125}$

552 frameshift allele. Gel shows products of PCR of gDNA from Slx $4^{+/+}$and $S l x 4^{\Delta 125 / \Delta}$ clones. Red

553 letters above sequencing chromatogram indicate frame shift. c. Proliferative competition assay in

554 presence of Mitomycin $\mathrm{C}(\mathrm{MMC})$, measuring enrichment of $\mathrm{GFP}^{+}$wild type vs. $\mathrm{GFP}^{-}$test

555 culture. Data, normalized to $0 \mathrm{ng} / \mathrm{mL}$ MMC, shows mean $(\mathrm{n}=3)$ and standard deviation. $\mathbf{d}$.

556 Representative raw FACS data (uncorrected for transfection efficiency) for one $S l x 4^{+/+}$(\#3) and

557 one $S l x 4^{\Delta 125 / \Delta}(\# 40) 6 x$ Ter-HR reporter clone co-transfected with either empty vector (EV), I-

558 SceI or Tus expression vectors as shown. FACS plots produced from pooled technical duplicates,

$559 \mathrm{n}=6$. Numbers indicate percentages. e and f. Tus/Ter-induced STGC (e) and LTGC (f) in $S l x 4^{+/+}$

560 clones and Slx $4^{\Delta 125 / \Delta}$ clones. $\mathbf{g}$ and $\mathbf{h}$. I-SceI-induced STGC (g) and LTGC (h) in Slx $4^{+/+} v s$.

$561 S l x 4^{\Delta 125 / \Delta}$ clones. Data shows mean values, $\mathrm{n}=6$. Error bars: standard error of the mean (s.e.m). In

562 this and all subsequent figures: ${ }^{*} \mathrm{p}<0.05 ;{ }^{*} \mathrm{p}<0.01 ; * * * \mathrm{p}<0.001 ; *^{* * *} \mathrm{p}<0.0001$; ns, not 563 significant. 

stalled forks. a. Schematic of SLX4 protein. MLR: XPF-interacting domain. SAP: MUS81-

567 interacting domain. SBD: SLX1-interacting domain. b. Cell cycle distribution of $S l x 4^{+/-}$and $568 S l \times 4^{\Delta \mathrm{UBZ} /-}$ cells either untreated or treated with $20,30,40$ or $50 \mathrm{ng} / \mathrm{mL}$ of MMC. Data shows 569 mean values, $\mathrm{n}=3$. Error bars: s.e.m. Untreated samples were compared using the Student's $t$-test; 570 MMC-treated groups were compared by one-way ANOVA. $\mathbf{c}$ and $\mathbf{d}$. Tus/Ter-induced STGC (c) 571 and I-SceI induced STGC (d) in $S l x 4^{+/-} v s . S l x 4^{\Delta \mathrm{UBZ} /-}$ clones. Analysis by ANOVA, n=5. Error 572 bars: s.e.m. e and f. Impact of exogenous hSLX4 on Tus/Ter induced STGC (e) and I-SceI 573 induced STGC (f) in two independent $S l x 4^{+/-}$and two independent $S l x 4^{\Delta \mathrm{UBZ} /-}$ clones. EV: empty 574 vector. $4 \mathrm{C}>\mathrm{A}$ : hSLX4 with inactivated UBZ motifs. Analysis by Student's $t$-test, $\mathrm{n}=6$. Error bars: 575 s.e.m. g. Schematic of $S l x 4^{+/}$and $S l x 4^{\Delta \mathrm{UBZ} /-}$ tagged with a dual degron containing a C-terminal 576 8xHA tag. h. RT-qPCR analysis of Slx 4 mRNA in Slx $4^{\mathrm{deg} /-}$ and $S l x 4^{\Delta \mathrm{UBZ} \operatorname{deg} /-}$ clones. Data 577 normalized to Gapdh mRNA using the $2^{-\Delta \mathrm{CT}}$ method. Expression of $S l x 4^{\Delta \mathrm{UBZdeg} /-}$ normalized to $578 S l \times 4^{\operatorname{deg} /-}$. Analysis by Student's $t$-test (n=3). Error bars: standard deviation (s.d.). i. Anti-HA 579 immunoblot of HA-degron tagged SLX4, 24 hours after addition of degron-activating drugs 5580 IAA and/or Asv. Asv: Asunaprevir. 5-IAA: 5-adamantyl-IAA. H3: Histone H3 loading control. 581 j. Anti-HA ChIP analysis of SLX4-HA at Tus/Ter RFB in Slx $4^{\mathrm{deg} /-}$ and Slx $4^{\Delta \mathrm{UBZ} \operatorname{deg} /-}$ cells. 582 Analysis by Student's $t$-test $(\mathrm{n}=3)$. Error bars: s.d.

584 Fig. 3. Interactions of SLX4 with MUS81 and with SLX1 are dispensable for Tus/Ter585 induced HR. a. Schematic of SLX4 protein lacking the MUS81-interacting SAP domain. b and 586 c. Proliferative competition assay in presence of MMC (b), Olaparib (c), showing enrichment of 587 wild type $\mathrm{GFP}^{+}$cells against the genotypes shown. Data show mean of values normalized to 
untreated samples (n=3). Error bars: s.d. d and e. Tus/Ter-induced STGC (d) and I-SceI induced STGC (e) in $S l x 4^{+/-} v s . S l x 4^{\Delta \mathrm{SAP} /-}$ clones. Analysis by ANOVA, n=5. Error bars: s.e.m. f.

590 Schematic of SLX4 protein lacking the SLX1-interacting SBD domain. $\mathbf{g}$ and $\mathbf{h}$. Proliferative 591 competition assay in presence of $\operatorname{MMC}(\mathbf{g})$, Olaparib (h), showing enrichment of wild type GFP ${ }^{+}$ 592 cells against the genotypes shown. Data show mean of values normalized to untreated samples 593 (n=3). Error bars: s.d. (i and j). Tus/Ter-induced STGC (i) and I-SceI induced STGC (j) in $594 S l x 4^{+/-} v s . S l x 4^{4 \mathrm{SBD} /-}$ clones. Analysis by ANOVA, n=5. Error bars: s.e.m.

Fig. 4. Loss of the SLX4-XPF interaction causes a characteristic FA phenotype a. Schematic 597 of SLX4 protein lacking the XPF-interacting MLR domain. b. Cell cycle distribution of Slx $4^{+/-}$ 598 and $S l x 4^{\Delta \mathrm{MLR} /-}$ cells either untreated or treated with $20,30,40$ or $50 \mathrm{ng} / \mathrm{mL}$ of MMC. Data shows 599 mean values, $\mathrm{n}=3$. Error bars: s.e.m. Untreated samples were compared using the Student's $t$-test; 600 MMC-treated groups were compared by one-way ANOVA. c. Representative images of 601 metaphase spreads from Slx4 mutants indicated, showing chromatid breaks (black arrows) and 602 radial chromosomes (black arrowheads) in untreated cells and cells treated with $20 \mathrm{ng} / \mathrm{mL} \mathrm{MMC}$ 603 for $12 \mathrm{~h}$ (d) and (e). Quantitation of the number of breaks (d) and radial chromosomes (e) per 604 metaphase nucleus in $S l x 4^{+/-}, S l x 4^{\Delta \mathrm{UBZ} /-}$ and $S l x 4^{\Delta \mathrm{MLR} /-}$ cell lines. Fig. 5. The SLX4-XPF interaction is required for Tus/Ter-induced STGC. a and b Tus/Ter607 induced STGC (a) and I-SceI induced STGC (b) in Slx $4^{+/-} v s . S l x 4^{\Delta \mathrm{MLR} /-}$ clones. Analysis by 608 ANOVA, n=4. Error bars: s.e.m. c. Schematic showing mutants of human SLX4 used for 609 transient expression experiments. d. RT-qPCR analysis of expression of human SLX4 variants in 610 mES cells. SLX4 expression normalized to Gapdh and displayed as fold difference compared to 
611 empty vector (EV) of the same experiment, $\mathrm{n}=3$. e and f. Impact of $\mathrm{h} S L X 4$ variants on Tus/Ter612 induced STGC (e) and I-SceI-induced STGC (f), following transient SLX4 expression in Slx $4^{+/-}$ 613 and Slx $4^{\Delta \mathrm{UBZ} /-}$ Ter-HR reporter clones.

614

615 Fig. 6. The XPF nuclease domain is required for Tus/Ter- and I-SceI-induced STGC. a.

616 Schematic of wild type XPF protein and XPF mutant lacking the nuclease domain. b. Strategy

617 for generating $X p f^{\triangle \mathrm{Nuc}}$ allele. Red half-arrow heads: genotyping primers. Gel shows PCR

618 products on gDNA from $X p f^{4 /-}$ and $X p f^{\Delta \mathrm{Nuc} /-}$ clones and sequencing chromatogram showing the 619 breakpoints in the in-frame mutant (\#6) $\mathbf{c}$ and $\mathbf{d}$. Proliferative competition assay in presence of $620 \mathrm{MMC}(\mathbf{c})$ or Olaparib (d), showing enrichment of wild type $\mathrm{GFP}^{+}$cells against the genotypes 621 shown. Data show mean of values normalized to untreated samples $(n=3)$. Error bars: s.d. e. 622 Representative images of metaphase spreads showing chromatid breaks (black arrows) and radial 623 chromosomes (black arrowheads) from indicated $X p f$ mutant cells, either untreated or treated 624 with $20 \mathrm{ng} / \mathrm{mL} \mathrm{MMC} \mathrm{for} \mathrm{12h.} \mathrm{f} \mathrm{and} \mathrm{g.} \mathrm{Quantitation} \mathrm{of} \mathrm{the} \mathrm{number} \mathrm{of} \mathrm{chromatid} \mathrm{breaks} \mathrm{(f)} \mathrm{radial}$ 625 chromosomes (g) per metaphase in $X p f^{4 /-}(\# 6)$ and $X p f^{\triangle \mathrm{Nuc} /-(\# 1)}$ clones. $\mathbf{h}$ and $\mathbf{i}$. Tus/Ter-induced 626 STGC (h) and I-SceI induced STGC (i) in $X p f^{4 /-} v s . X p f^{\Delta N u c /-}$ clones. Analysis by ANOVA, $\mathrm{n}=6$. 627 Error bars: s.e.m.

629 Fig. 7. Model of SLX4 action in Tus/Ter-induced HR. a. In wild type cells, FA pathway 630 activation and asymmetric fork reversal at forks bidirectionally stalled at Tus/Ter RFB enables 631 SLX4 recruitment and its retention at the stall site, mediated by interactions of the SLX4 UBZ 632 domain with as yet unidentified ubiquitinated components at the stall site. SLX4 MLR domain 633 enables recruitment of XPF-ERCC1, which incises the stalled leading strand of at least one sister 
634 chromatid (incision $a$, red triangle). Note that the second incision (red triangle $b$ ), which would

635 be required for ICL unhooking, may be redundant for generation of a DSB at Tus/Ter, since

636 there is no ICL. The two-ended DSB generated by SLX4-XPF is repaired by conservative HR

637 (STGC). b. In cells lacking the SLX4 UBZ domain, retention of SLX4-XPF at the stall site is

638 impaired, leading to inefficient incision of the stalled forks and reduced DSB formation. There is

639 a corresponding defect in Tus/Ter-induced STGC. c. In cells lacking the SLX4 MLR domain, or

640 lacking the XPF nuclease domain, XPF-mediated incision of the stalled forks is abolished,

641 resulting in a severe defect in Tus/Ter-induced STGC.

642

643 Methods

644

645 Molecular biology and sgRNA oligos

646 The 6xTer-HR reporters used were assembled using standard cloning methods described

647 previously for the $6 \times$ Ter-HR reporter ${ }^{27,28}$. Derivation of Tus and I-SceI expression plasmid are 648 described in ${ }^{27,59}$. Ter-containing plasmids were amplified in JJC33 (Tus ${ }^{-}$) strains of E. coli. All 649 plasmids used for transfection were prepared by endotoxin-free maxiprep (QIAGEN Sciences).

650 All primers used for genotyping, RT-qPCR, sgRNA synthesis, and chromatin

651 immunoprecipitation were purchased from Life Technologies.

652

653 Generation of mouse $S l x 4$ and $X p f$ mutant cell lines and cell culture

654 Slx4 mutant cell lines were derived from a conditional mouse $B r c a l^{\text {flexon11 }} \mathrm{Ter}$-HR reporter ES

655 cell line ${ }^{27}$. This founder cell line contains a single copy of the 6x Ter-HR reporter cassette,

656 targeted to the Rosa26 locus and verified by southern blot. Mouse ES cells are routinely thawed 
657 onto plates coated with mouse embryonic fibroblast (MEF) feeders, maintained in ES medium on 658 gelatinized plates, and regularly tested for mycoplasma infection by Myco-Alert assay (Lonza).

659 Slx4 mutant cell lines were generated using CRISPR/Cas9 mediated mutation of the Slx4 locus.

660 sgRNAs targeting Cas9 to the Slx4 locus were transcribed in vitro using the Engen sgRNA

661 Synthesis kit (New England Biolabs E3322S), purified using the RNA Clean and Concentrator

662 Kit (Zymo Research, R1017), and verified by denaturing 10\% TBE-urea PAGE. Cas9-sgRNA

663 RNP was pre-assembled in vitro in OptiMEM by mixing Spy NLS Cas9 (New England Biolabs,

664 M0646T) and purified sgRNA. Cells were co-transfected with either $0.45 \mu \mathrm{g}$ Cas9(1.1)

665 expression plasmid and $0.05 \mu \mathrm{g}$ of each purified sgRNA using Lipofectamine 2000 (Invitrogen).

666 After $72 \mathrm{hr}$, transfected cells were plated onto 6-cm dishes containing feeder MEFs without

667 selection. Individual clones were picked for expansion between 9 and 14 days later and Slx 4

668 mutant clones were identified by PCR and confirmed by sequencing. Slx 4 targeting sgRNA

669 sequences including PAM: Slx $4^{\Delta 125 / \Delta} 5^{\prime}$ exon 2 (5' CCT TCC TGT TTC ACG ACA ACT GG

670 3'), Slx $4^{\Delta 125 / \Delta} 3^{\prime}$ exon 2 (5' ATC CCC CCA AAA GAC TGC ACT GG 3'), Slx4 hemizygote

671 exon 2 (5’ ATC CCC CCA AAA GAC TGC ACT GG 3'), Slx4 hemizygote exon 15 (5’ ATC

672 CTT TTT CTT TCG GC CTG AGG 3'); Slx4 $\triangle U B Z$ 5' (5' ATG ACA GCC TGG AGG AGA

673 AGG GG 3'), Slx4 $\triangle U B Z$ 3' (5' ACA GCC TGA AGC AGG AGT TGG GG 3'); Slx4 $\triangle M L R$ 5'

674 (5' CCA AAA GGG AGC CAA GGA GGA GG 3'), Slx4 $\triangle M L R$ 3' (5' GGG CAT TGG GAC

675 ACA ATT GGA GG 3'); Slx4 $\triangle S A P$ 5' (5' CCA GAA CTC GGA AGC CAG ACA GG 3'), Slx4

$676 \Delta S A P 3^{\prime}$ ' (5' GGT AGT TCT GCG GGT ATC TGG GG 3'); Slx4 $\triangle S B D$ 5' (5' CGC AGA ACT

677 ACC TTG TCG CCA GG 3'), Slx4 $\triangle S B D$ 3' (5' GAA AAA GGA TCA GAA ATG ACA GG

678 3'); sgRNA targeting Slx4 dual degron (5' ATC CTT TTT CTT TCG GCC TGA GG 3'); Xpf

679 hemizygote 5' UTR (5' GTG CGG CCG CGG TCC GAA GGG 3'), Xpf hemizygote exon 11 (5' 


\section{Recombination assays}

685 These assays were performed as described in detail in ${ }^{76}$. Briefly, $1.6 \times 10^{5}$ cells were cotransfected in suspension with $0.5 \mathrm{mg}$ empty vector, pcDNA3b-myc NLS-Tus, or pcDNA3b-myc

687 NLS-I-SceI using Lipofectamine 2000 (Invitrogen). GFP+RFP-, GFP+RFP+ and GFP-RFP+

688 frequencies were scored 72 hours after transfection by flow cytometry Beckman Coulter

689 CytoFlex LX. For each duplicate sample condition, 3-6 x $10^{5}$ total events were scored. Repair

690 frequencies presented are corrected for background events and for transfection efficiency (50-

691 85\%). Transfection efficiency was measured by parallel transfection with $0.05 \mathrm{mg}$ wild type GFP

692 expression vector and $0.45 \mathrm{mg}$ empty vector. For transient hSLX4 rescue experiments, $1.6 \times 10^{5}$

693 cells were co-transfected in suspension with $0.4 \mathrm{mg}$ empty vector, pcDNA3b-myc NLS-Tus ${ }^{27}$,

694 or pcDNA3b-myc NLS-I-SceI ${ }^{59}$, and either $0.1 \mathrm{mg}$ empty vector and pHIV- 3xFLAG-AttB1-

695 hSlx4-AttB2- NatR-T2A-hCD52 and all other Slx4 domain mutant derivatives using

696 Lipofectamine 2000.

698 Competition assays

700 For all competition experiments, $1.6 \times 10^{5}$ cells were co-transfected in suspension with $0.45 \mathrm{mg}$

701 empty vector and either $0.05 \mathrm{mg}$ empty vector or $0.05 \mathrm{mg}$ GFP-expression plasmid using

702 Lipofectamine 2000 (Invitrogen). 18 hours after transfection, cells were counted, mixed 4:1, 
703 uncolored vs. GFP + marked cells, and $5 \times 10^{4}$ cells plated in triplicate. 6 hours after cell plating

704 growth medium was replaced with media containing MMC (Millipore Sigma Cat\#475820-

705 10MG). After two days incubation, GFP+ frequencies were scored on a Beckman Coulter

706 CytoFlex LX. Fold enrichment of cultures transiently co-transfected with GFP-expression

707 plasmid normalized to $0 \mathrm{mg} / \mathrm{mL}$ MMC control. Plots represent the mean of triplicate samples

708 from three independent experiments $(\mathrm{n}=3)$.

709

\section{RT-qPCR}

711 RNA extraction was performed using QIAGEN RNeasy Mini Kit (QIAGEN Sciences,). All

712 analyses of Gapdh and genes of interest were performed using an Applied Biosystems 7300 Real

713 time PCR System using Power SYBR Green RNA-to CT ${ }^{\mathrm{TM}}$ 1-Step Kit (Applied Biosystems,

714 Foster City, CA).Primers for RT-PCR: Gapdh sense (5' CGT CCC GTA GAC AAA ATG GT

715 3'); Gapdh antisense (5' TCG TTG ATG GCA ACA ATC TC 3'); Slx4 exon2 frameshift sense

716 (5' CAG AGA CAC CAA AAG GTG CTA ACC 3); Slx4 exon2 frameshift antisense (5' GCA

717 CGG ACT CCA AAC CTG TCC AGT 3'); Slx4 hemizygote sense (5' CAG AGA CAC CAA

718 AAG GTG CTA ACC 3'); Slx4 hemizygote antisense (5' GCA CGG ACT CCA AAC CTG TCC

719 AGT 3'); Slx4-UBZ sense (5' GCA ACA TGT GAA CAG ATG CCT); Slx4-UBZ antisense (5'

720 TTG GTG GTG AGG AAC GGT TT 3'); Slx4-MLR sense (5’ ACC ACC AAA AGG GAG

721 CCA AG 3'); Slx4-MLR antisense (5' CTT GCG ACT TTT CTT CTC TGC T 3'); Slx4-SAP

722 sense (5' CAG AGA CAC CAA AAG GTG CTA ACC 3'); Slx4-SAP antisense (5' GCA CGG

723 ACT CCA AAC CTG TCC AGT 3'); Slx4-SBD sense (5' CTG CAA CCC TTC AAG GTT GCC

724 AAC 3'); Slx4-SBD antisense (5' GAG GAG CTT TTA GAG CTA AAG GAG 3'), Xpf

725 hemizygote sense (5' GGG AAA CCT CTG AGG GTT TAC TTT 3'), Xpf hemizygote antisense 
726 (5' CTA GCT TTT TCT CTT ATG AGC TTT 3'), Xpf helicase sense (5' GTC AGA ATT CAG

727 GTT GGC TTT TCC 3'); Xpf helicase antisense-(5' TTG TTT CTT GAA CTT CTG GAC TCG

728 3'), Xpf nuclease sense (5' GAA AAG CTC ATA AGA GAA AAA GCT 3'); Xpf nuclease

729 antisense-(5' CTC CTG GCC ACC GGC TTT CCG AGT 3'); Xpf Hhh2 sense (5' GAA GAT

730 GCC TGG GGT CAA CGC CAA 3'); Xpf Hhh2 antisense-( 5’ AGG AAG TCG TGC AGC

731 TGC TTG GCA T 3'). N-terminal FLAG tagged human SLX4 sense (5’ ATG TGA ACA GGT

732 GCT TGG ATG AAG 3'), antisense (5’ ATC ACT GAA GCT GAA CAT GGG TGG 3’).

733 mRNA levels were measured in technical triplicates. Target gene expression level was

734 normalized to Gapdh using the $2^{-\Delta \mathrm{CT}}$ method and the mutants were normalized to the

735 corresponding wild-type.

736

737 Isolation of Chromatin-bound SIx4 and ERCC1 western blot

$738 \sim 10 \times 10^{6}$ cells were freshly harvested and washed with 1x PBS and lysed in 5 volumes of chilled

739 A1 buffer (50mM HEPES, 140mM Nacl,1mMEDTA,10\%glycerol,0.5\% NP-40,0.25\% TritonX-

$740100,1 \mathrm{mM}$ DTT, and 1x protease inhibitor). Each lysate was centrifuged at $1,100 \times \mathrm{g}$ at $4{ }^{\circ} \mathrm{C}$ for 2

741 min and the supernatant was discarded. Pellets were resuspended by gentle pipetting in A1 buffer

742 and samples were incubated for 10 minutes on ice. Each suspension was centrifuged at 1,100 x g

743 at $4{ }^{\circ} \mathrm{C}$ for $2 \mathrm{~min}$ and the supernatant again discarded. Pellets were resuspended by gentle

744 pipetting in 2 volumes of ice cold E2 buffer (10mM Tris-HCl pH-8,200mMNacl, 1mM EDTA

745 and $0.5 \mathrm{mM}$ EGTA, and 1x Protease inhibitor). Each suspension was centrifuged at 1,100 $\mathrm{x} g$ at 4

$746{ }^{\circ} \mathrm{C}$ for $2 \mathrm{~min}$ and the supernatant discarded. Pellets were resuspended by gentle pipetting with A1

747 buffer and incubated for 10 minutes on ice. Each suspension was centrifuged at $1,100 \times \mathrm{g}$ at $4{ }^{\circ} \mathrm{C}$

748 for 2 min and the supernatant discarded. Pellets were resuspended by gentle pipetting in 2 
volumes of ice cold E3 buffer (500mM Tris- $\mathrm{HCl}$ pH-6.8, 500mMNacl and 1x Protease inhibitor.

750 Each sample was sonicated in a water bath using Diagenode Bioruptor 300 with attached $4^{\circ} \mathrm{C}$

751 chiller cycling $30 \mathrm{sec}$ on and $30 \mathrm{sec}$ off on high setting for $5 \mathrm{~min}$. Sonicated samples were

752 centrifuged at $16,000 \mathrm{xg}$ for $15 \mathrm{~min}$ at $4^{\circ} \mathrm{C}$. The supernatant corresponding to the chromatin

753 fraction was transferred and subjected for western blot analysis using following antibodies; anti-

754 HA (Abcam ab9110, 1:1000), anti-ERCC1 (Santacruz sc-17809, 1:250), Histone H3 (Abcam 755 ab1791, 1:3000).

757 Chromatin immunoprecipitation Assay

758 Chromatin immunoprecipitation was performed as described previously ${ }^{77}$. Transfection of $2.0 \mathrm{x}$

$75910^{5} \mathrm{Ter}$-HR reporter cells of the genotypes noted was performed with $0.5 \mu \mathrm{g}$ empty vector, 760 pcDNA3 $\beta$-MYC NLS-I-SceI or pcDNA3ß-MYC NLS-Tus-F140A-3xFlag using Lipofectamine 7612000 (Invitrogen). 10 million cells were collected 24 hours after transfection and fixed in 1\% 762 formaldehyde supplemented serum free media. Fixation was quenched by addition of glycine to

$763125 \mathrm{mM}$. Cells were lysed in lysis buffer (0.1\% SDS, $20 \mathrm{mM}$ EDTA, $50 \mathrm{mM}$ Tris $\mathrm{pH} 8.1)$

764 containing protease inhibitor (Roche protease inhibitor, Roche 13539320). Chromatin shearing to

$765 \sim 500$ bp was accomplished using Diagenode Bioruptor 300 with attached $4^{\circ} \mathrm{C}$ chiller for 20

766 cycles, 15 seconds on and 30 seconds off at medium setting. To avoid non-specific binding to

767 protein A/G beads, $100 \mu \mathrm{l}$ lysates for each ChIP sample were precleared by the addition of $10 \mu \mathrm{l}$

768 activated Magna ChIP magnetic beads (Millipore Sigma, 16-663) in ChIP dilution buffer (1\%

769 Triton-X-100, 2mM EDTA, $150 \mathrm{mM} \mathrm{NaCl}, 20 \mathrm{mM}$ Tris $\mathrm{pH} 8.1$ ) and incubation for $1 \mathrm{hr}$ at $4^{\circ} \mathrm{C}$

770 with gentle mixing. After removal of beads by magnet, for each immunoprecipitation, $7 \mu 1$ of 771 anti-HA (Abcam ab9110) was added and mixed for 12 hours at $4^{\circ} \mathrm{C}$ followed by addition of $10 \mu \mathrm{l}$ 
772 activated Magna ChIP beads and mixing for 16 hours at $4^{\circ} \mathrm{C}$. Beads were washed six times in

773 ice-cold ChIP RIPA buffer (50mM HEPES pH 7.6, $1 \mathrm{mM}$ EDTA, $7 \mathrm{mg} / \mathrm{mL}$ sodium

774 deoxycholate, 1\% NP-40) followed by two washes in ice-cold TE (10 mM Tris pH8.0, $1 \mathrm{mM}$

775 EDTA). Crosslinks were reversed and DNA was eluted by incubation in $100 \mu$ L Elution buffer

$776\left(1 \% \mathrm{SDS}, 200 \mathrm{mM}\right.$ sodium bicarbonate, $5.6 \mu \mathrm{g} / \mathrm{mL}$ RNAse A) overnight at $65^{\circ} \mathrm{C}$. Protein was

777 removed by proteinase $\mathrm{K}$ digest for $30 \mathrm{~min}$ at $55^{\circ} \mathrm{C}$. Released DNA was purified by Qiagen PCR

778 Purification column (Qiagen, 28106) and analyzed by qPCR on an ABI Prism 7300 or

779 QuantStudio3 using SYBR Green (Applied Biosystems, 4368702). Primers for qPCR: +109 bp

780 sense (TCC GGA TAG GGA TAA CAG GGT A); +109 bp antisense (GTC GGC CAT GAT

781 ATA GAC GTT G); 128 bp sense (GAG CGC ACC ATC TTC TTC A); 128 bp antisense (TCC

782 CTA CGA TGC CCT TCA). Data are presented as the mean calculated from three independent

783 experiments $(\mathrm{n}=3)$ normalized against untreated controls (empty vector) and control locus

784 (beta-actin) using the $2^{-\Delta \mathrm{CT}}$ method ${ }^{78}$.

785

\section{Cell Cycle Analysis}

787 ES cells were grown on a 6 -well plate until $\sim 70 \%$ confluent. Cells were treated with varying 788 concentrations of MMC for $12 \mathrm{~h}$ and compared with untreated controls. After $12 \mathrm{~h}$, cells were 789 pulsed with $10 \mu \mathrm{M}$ BrdU for 15 min. Harvested cells were then washed with pre-chilled 1x PBS

$790 \mathrm{pH} 7.0$ by gently pipetting it and spun at $1000 \mathrm{rpm}$ for 5 mins. Supernatant was discarded and the

791 pellet was gently resuspended in $50 \mu 1$ PBS. Cells were fixed by adding $5 \mathrm{~mL}$ of chilled $70 \%$

792 Ethanol in a dropwise manner while gently but constantly vortexing to prevent clumping. Fixed

793 cells are stored at $4^{\circ} \mathrm{C}$ in the dark. Before immunostaining for BrdU, cells were spun at 1000rpm

794 for 5 mins to remove the ethanol. While gently vortexing, $0.5 \mathrm{~mL}$ of $2 \mathrm{M} \mathrm{HCl}, 0.5 \%$ Triton-X-100 
795 solution was added in a dropwise manner to denature DNA. Cells were incubated at RT for 30

796 mins, with intermittently vortexing approximately every 10 mins. Excess acid was removed by

797 spinning and cells were neutralized using $1 \mathrm{~mL}$ 0.1M Sodium Borate Decahydrate. Once the

798 neutralizing solution is removed, resuspend pellet in $50-100 \mu 1$ of $1 \mathrm{xPBS}$ containing $1 \% \mathrm{BSA}$.

799 BrdU was counterstained using anti-BrdU antibody (Abcam ab8039, 1:100) and cells were

800 incubated at RT for 30 mins in the dark. Cells were then washed with $5 \mathrm{~mL}$ chilled $1 \mathrm{xPBS}$

801 containing $20 \mathrm{mM}$ HEPES $\mathrm{pH} 7.4,0.5 \%$ tween-20 to remove excess antibody. A secondary

802 FITC-conjugated rabbit anti-mouse antibody (Jackson Immunoresearch, 115-095-146-1:50) was

803 used and cells were incubated at RT for 30 mins in the dark. Cells were then washed with $5 \mathrm{~mL}$

804 chilled 1x PBS containing 20mM HEPES pH 7.4, 0.5\% tween-20 to remove secondary antibody.

805 Cells were resuspended with $300 \mu 1$ of solution containing $38 \mathrm{mM}$ sodium citrate, $69 \mu \mathrm{M}$

806 propidium iodide, $5 \mu 1 / \mathrm{mL}$ RNaseA in the dark for 15 mins at $37^{\circ} \mathrm{C}$. Approximately $10^{4}$ events

807 were acquired using Cytoflex LX and the results were analyzed using FloJo software.

808

809 Chromosomal breakage assay

$810 \sim 3 \times 10^{6}$ adherent cells were either untreated or treated with $0.02 \mu \mathrm{g} / \mathrm{mL}$ MMC for $12 \mathrm{~h}$. Cells

811 were arrested with colcemid ( $0.17 \mu \mathrm{g}$ per $\mathrm{ml}$ of media) for $20 \mathrm{~min}$. Cells were harvested and

812 incubated at $37^{\circ} \mathrm{C}$ in $0.075 \mathrm{M} \mathrm{KCl}$ for 10 mins and fixed in freshly prepared methanol:glacial

813 acidic acid $(3: 1 \mathrm{vol} / \mathrm{vol})$. Cells were stored at $4^{\circ} \mathrm{C}$ at least overnight. Cells were dropped onto wet

814 slides and air dried at $40^{\circ} \mathrm{C}$ for 60 min before staining with KaryoMAX Giemsa (Invitrogen)

815 Gurr Buffer for 3 min. After rinsing with fresh Gurr Buffer followed by distilled water, the slides

816 were fully dried at $40{ }^{\circ} \mathrm{C}$ for $60 \mathrm{~min}$ and scanned using the Metasystems Metafer application. 
817 The experiments were randomized and breaks, and radial chromosomes were scored by a person

818 blinded to the identity of the samples.

\section{Statistical methods}

821 Data shown represents the arithmetic mean and error bars represent either the standard error of

822 the mean (s.e.m.) or standard deviation (s.d.) of between three $(n=3)$ and nine $(n=9)$

823 independent experiments (as noted in each Figure Legend). Data points for each independent

824 experiment were typically collected as the mean of technical duplicates. This mean value was

825 taken as the solitary data point for that individual experiment. The arithmetic mean of samples

826 collected for groups of independent experiments for repair frequency statistical analysis, was

827 calculated and data points for each independent experiment used to calculate the mean and

828 s.e.m., calculated as standard deviation $/ \sqrt{ } \mathrm{n}$, (n indicates the number of independent

829 experiments). Differences between sample pairs repair frequencies and fold enrichment for ChIP

830 were analyzed by Student's two-tailed unpaired $t$-test, assuming unequal variance. One-way

831 ANOVA statistical analysis of greater than three samples was performed when indicated. P-

832 values are indicated in the figure and the P-value code for each figure is as written in the Fig. 1

833 legend. No statistical methods were used to predetermine sample size. The experiments were not

834 randomized, and investigators were not blinded to allocation during experiments and outcome

835 assessment. All statistics were performed using GraphPad Prism v7.0d software.

\section{Supplementary Figure Legends}


839 Supplementary Fig. 1. Characterization of $S l x 4^{\Delta 125 / \Delta}$ cells. a. RT qPCR analysis of $S l x 4$

840 mRNA normalized to Gapdh mRNA using the $2^{-\Delta C T}$ method in three independent experiments

841 ( $\mathrm{n}=3$ ). Error bars, standard deviation (s.d.). b and c. Ratio of Tus/Ter-induced (b) and I-SceI-

842 induced (c) LTGC: total HR in $S l x 4^{+/+}$clones and $S l x 4^{\Delta 125 / \Delta}$ clones. d. CRISPR/Cas9 with dual

843 sgRNA targeting to generate $S l x 4^{+/-}$hemizygous cells. The $S l x 4^{-}$allele contains a $19.3 \mathrm{~kb}$

844 deletion between exons 2 and 15. Red half arrowheads: PCR and sequencing primers specific to

845 Exons 2 and 15. Predicted PCR product sizes for $S l x 4^{+}$(wild type) allele shown. e. Gel shows

846 PCR products detected using gDNA from $S l x 4^{+/+}$and $S l x 4^{+/-}$clones. Note the $444 \mathrm{bp} \mathrm{PCR}$

847 product formed across the $19.3 \mathrm{~kb}$ deletion between exons 2 and 15 in $S l x 4^{+/}$cells. f. DNA

848 sequencing chromatogram of PCR products from (e). g. RT qPCR analysis of Slx4 mRNA in

$849 S l \times 4^{+/+}$and $S l x 4^{+/-}$cells. Data shows mean of three independent experiments $(\mathrm{n}=3)$ normalized to

850 Gapdh mRNA using the $2^{-\Delta \Delta C T}$ method and analyzed by Student's $t$-test. Error bars: s.d.. h.

$851 \mathrm{Tus} / \mathrm{Ter}$-induced and I-SceI-induced repair frequencies in $S l \times 4^{+/+}$and $S l x 4^{+/-}$cells. In this and all

852 subsequent Supplemental Figures: ${ }^{*} \mathrm{p}<0.05 ;{ }^{* *} \mathrm{p}<0.01 ;{ }^{* * *} \mathrm{p}<0.001 ;{ }^{* * * *} \mathrm{p}<0.0001$; ns, not

853 significant.

855 Supplementary Fig. 2. The SLX4 UBZ domain mediates I-SceI-induced LTGC. a. Strategy

856 for in-frame deletion of UBZ domain-encoding regions of Slx4. Red half-arrow heads:

857 genotyping PCR primers. Gel shows PCR products using gDNA from $S l x 4^{+/-}$and $S l x 4^{\mathrm{AUBZ} /-}$

858 clones. Sequencing chromatogram shows in-frame breakpoint of $S l x 4^{\Delta \mathrm{UBZ}}$ allele. b. RT-qPCR

859 analysis of mRNA encoding UBZ, MLR, SAP and SBD domains in $S l x 4^{+/-}$and $S l \times 4^{\Delta \mathrm{UBZ} /-}$ clones.

860 Data normalized to Gapdh mRNA using the $2^{-\Delta \mathrm{CT}}$ method, mRNA expression in Slx $4^{\Delta \mathrm{UBZ} /-}$

861 samples were normalized to $S l x 4^{+/-}$of the same experiment and analyzed by Student's $t$-test 
$862(\mathrm{n}=3)$. Error bars: standard deviation. $\mathrm{P}=0.0125$ for mRNA expression of the UBZ region in

$863 S l x 4^{+/-}$compared to $S l x 4^{\mathrm{AUBZ} /-}$. No significant differences were observed in expression levels of

864 all other domains. $\mathbf{c}$ and $\mathbf{d}$. Proliferative competition assay in presence of MMC (c) or Olaparib

865 (d), showing enrichment of wild type $\mathrm{GFP}^{+}$cells against the genotypes shown. Data show mean

866 of values normalized to untreated samples ( $n=3)$. Error bars: s.d. e. RT-qPCR analysis of N-

867 terminal Flag-tagged wild-type full-length human SLX4 and UBZ 4C $>$ A expression plasmids

$86848 \mathrm{~h}$ after transfection. Data normalized to Gapdh mRNA using the $2^{-\Delta \mathrm{CT}}$ method and compared

869 to empty vector (EV) control. Error bar: s.d. f and g. Tus/Ter-induced LTGC (f) and LTGC/Total

870 HR ratio (g) in $S l x 4^{+/-}$and $S l x 4^{\Delta \mathrm{UBZ} /-}$ clones. Analysis by ANOVA, n=5. Error bars: s.e.m. h.

871 Impact of exogenous hSLX4 on Tus/Ter-induced LTGC in two independent $S l \times 4^{+/-}$and two

872 independent Slx $4^{\Delta \mathrm{UBZ} /-}$ clones. EV: empty vector. $4 \mathrm{C}>\mathrm{A}$ : $\mathrm{h} S L X 4$ with inactivated UBZ motifs.

873 Analysis by Student's $t$-test, $\mathrm{n}=6$. Error bars: s.e.m. i and j. I-SceI-induced LTGC (i) and

874 LTGC/Total HR ratio (j) in $S l x 4^{+/-}$and $S l x 4^{\Delta \mathrm{UBZ} /-}$ clones. Analysis by ANOVA, $\mathrm{n}=5$. Error bars:

875 s.e.m. k. Impact of exogenous hSLX4 on I-SceI-induced LTGC in two independent $S l x 4^{+/-}$and

876 two independent $S l x 4^{\Delta \mathrm{UBZ} /-}$ clones. EV: empty vector. $4 \mathrm{C}>\mathrm{A}$ : hSLX 4 with inactivated UBZ

877 motifs. Analysis by Student's $t$-test, $\mathrm{n}=6$. Error bars: s.e.m.

$878 \quad$.

879 Supplementary Fig. 3. Characterization of $S l x 4^{\Delta \mathrm{SAP} /-}$ and $S l x 4^{\Delta \mathrm{SBD} /-}$ mutant clones. a.

880 Strategy for in-frame deletion of SAP domain-encoding region of Slx4. Red half-arrow heads:

881 genotyping PCR primers. Gels show PCR products using gDNA from $S l x 4^{+/-}$and $S l x 4^{\Delta \mathrm{SAP} /-}$

882 clones. Sequencing chromatogram shows in-frame breakpoint of $S l x 4^{\Delta \mathrm{SAP}}$ allele. b. RT-qPCR

883 analysis of UBZ, MLR, SAP and SBD domains encoding mRNA in $S l x 4^{+/-}$and $S l x 4^{\mathrm{ASAP} /}$-clones.

884 Data normalized to Gapdh mRNA using the $2^{-\Delta \mathrm{CT}}$ method, mRNA expression in $S l x 4^{\Delta \mathrm{SAP} /-}$ were 
normalized to $S l x 4^{+/}(\mathrm{n}=3)$ of the same experiment and analyzed by Student's $t$-test. Error bars: s.d. $\mathrm{P}=0.0478$ for mRNA expression of the SAP region in $S l x 4^{+/-}$compared to $S l x 4^{\Delta \mathrm{SAP} /}{ }^{\text {. No }}$

887 significant differences were observed in expression levels of all other domains. $\mathbf{c}$ and $\mathbf{d}$. Tus/Ter888 induced LTGC (c) and I-SceI-induced LTGC (d) in $S l x 4^{+/-}$and $S l x 4^{4 \mathrm{SAP} /-}$ clones. Analysis by ANOVA, n=5. Error bars: s.e.m. e. Strategy for in-frame deletion of SBD domain-encoding 890 region of Slx4. Red half-arrow heads: genotyping PCR primers. Gels show PCR products using 891 gDNA from $S l x 4^{+/-}$and $S l x 4^{4 \mathrm{SBD} /-}$ clones. Sequencing chromatogram shows in-frame breakpoint 892 of $S l x 4^{\mathrm{SBD}}$ allele. f. RT-qPCR analysis of UBZ, MLR, SAP and SBD domains encoding mRNA 893 in $S l x 4^{+/-}$and $S l x 4^{\Delta \mathrm{SBD} /-}$ clones. Data normalized to Gapdh mRNA using the $2^{-\Delta \mathrm{CT}}$ method, 894 mRNA expression in $S l x 4^{\mathrm{SBD} /-}$ was normalized to $S l \times 4^{+/-}$of the same experiment and analyzed 895 by Student's $t$-test ( $\mathrm{n}=3$ ). Error bars: standard deviation. $\mathrm{P}=0.048$ for mRNA expression of the SAP region in $S l x 4^{+/-}$compared to $S l x 4^{\Delta \mathrm{SBD} /-}$. No significant differences were observed in 897 expression levels of all other domains. $\mathbf{g}$ and $\mathbf{h}$. Tus/Ter-induced LTGC (c) and I-SceI-induced LTGC (d) in $S l x 4^{+/-}$and $S l x 4^{\Delta \mathrm{SBD} /-}$ clones. Analysis by ANOVA, n=5. Error bars: s.e.m.

900 Supplementary Fig. 4. Characterization of $S l x 4^{\Delta \mathrm{MLR} /-}$ mutant clones. a. Strategy for in-frame 901 deletion of MLR domain-encoding region of Slx4. Red half-arrow heads: genotyping PCR 902 primers. Gels show PCR products using gDNA from $S l x 4^{+/-}$and $S l x 4^{\Delta \mathrm{MLR} /-}$ clones. Sequencing 903 chromatogram shows in-frame breakpoint of $S l x 4^{\triangle \mathrm{MLR}}$ allele. b. RT-qPCR analysis of UBZ, 904 MLR, SAP and SBD domains encoding mRNA in $S l x 4^{+/-}$and $S l \times 4^{\Delta \mathrm{MLR} /-}$ clones. Data normalized 905 to Gapdh mRNA using the $2^{-\Delta C \mathrm{~T}}$ method, mRNA expression in $S l \times 4^{\Delta \mathrm{MLR} /}$ were normalized to $906 S \mathrm{Sl} \times 4^{+/-}$of the same experiment and analyzed by Student's $t$-test $(\mathrm{n}=3)$. Error bars: standard 907 deviation (s.d.). $\mathrm{P}=0.01$ for mRNA expression of the MLR region in $S l \times 4^{+/-}$compared to 
$908 S l x 4^{\Delta \mathrm{MLR} /-}$. No significant differences were observed in expression levels of all other domains. c

909 and d. Proliferative competition assay in presence of MMC (c) or Olaparib (d), showing

910 enrichment of wild type $\mathrm{GFP}^{+}$cells against the genotypes shown. Data show mean of values

911 normalized to untreated samples $(n=3)$. Error bars: s.d. e. Representative images of metaphase

912 spreads showing chromatid breaks (black arrows) and radial chromosomes (black arrowheads) in

913 cells either untreated or treated with $20 \mathrm{ng} / \mathrm{mL}$ MMC for $12 \mathrm{~h}$ in $S l x 4^{\Delta \mathrm{SAP} /-}$ and $S l x 4^{\Delta \mathrm{SBD} /-}$ cells. f

914 and g. Quantitation of the number of breaks (f) and radial chromosomes (g) per metaphase

915 nucleus in Slx4 mutant cells shown.

916

917 Supplementary Fig. 5. The SLX4 MLR domain contributes to I-SceI-induced LTGC. a and

918 b. Tus/Ter-induced LTGC (a) and I-SceI-induced LTGC (b) in Slx $4^{+/-}$and Slx $4^{\Delta \mathrm{MLR} /-}$ clones.

919 Analysis by ANOVA, n=5. Error bars: s.e.m. c and d. Impact of exogenous hSLX4 alleles (see

920 Fig. 5c) on Tus/Ter-induced LTGC (c) and on I-SceI-induced LTGC (d) in Slx $4^{+/-}$and Slx $4^{\Delta \mathrm{UBZ} /-}$

921 clones. EV: empty vector. 4C>A: hSLX4 with inactivated UBZ motifs. Analysis by Student's $t$ -

922 test, $\mathrm{n}=6$. Error bars: s.e.m.

923

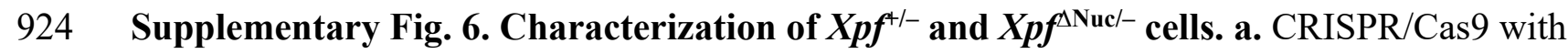

925 dual sgRNA strategy for generation of $X p f^{4-}$ hemizygous cells. The $X p f$ allele contains a 42.3

$926 \mathrm{~kb}$ deletion between the 5'UTR and exon 11. Red half arrowheads: PCR and sequencing primers

927 specific to 5'UTR and Exon 11. Predicted PCR product sizes for $X p f^{+}$(wild type) allele shown.

928 b. Gel shows PCR products using gDNA from $X p f^{f^{\prime+}}$ and $X p f^{+/-}$clones. Note the 440 bp PCR

929 product formed across the $42.3 \mathrm{~kb}$ deletion between the 5'UTR and exon $11 \mathrm{in} X p f^{4 /-}$ cells. c.

930 Sequencing chromatogram of PCR products from (b), showing breakpoint sequence of Xpf 
931 allele and intact sgRNA-targeted sequences of $X p f^{+}$allele. Underlined base in the $X p f^{-}$sequence

932 denotes a single base-pair insertion at the breakpoint generated during CRISPR/Cas9 treatment.

933 d. RT qPCR analysis of mRNA in $X p f^{f^{1+}}$ and $X p f^{+/-}$cells. Data shows mean of three independent

934 experiments $(\mathrm{n}=3)$ normalized to Gapdh mRNA using the $2^{-\Delta \Delta \mathrm{CT}}$ method and analyzed by

935 Student's $t$-test. Error bars: s.d. e. Tus/Ter-induced and I-SceI-induced HR frequencies in Xpf ${ }^{+/+}$

936 or $X p f^{+-}$clones. Analysis by Student's $t$-test, $\mathrm{n}=4$. Error bars: s.e.m. f. RT-qPCR analysis of

937 mRNA encoding the XPF helicase, nuclease and Hh2h domains in 4 isogenic $\mathrm{Xpf}^{+/-}$clones and 4

$938 X p f^{\Delta \mathrm{Nuc} /}$ clones. Data normalized to Gapdh mRNA using the $2^{-\Delta \mathrm{CT}}$ method, mRNA expression

939 in $X p f^{\Delta \mathrm{Nuc} /-}$ was normalized to $X p f^{+/-}$of the same experiment and analyzed by Student's $t$-test

$940(\mathrm{n}=3)$. Error bars: s.d. $\mathrm{P}=0.0153$ for mRNA expression of the Nuclease region in $X p f^{+/-}$

941 compared to $X p f^{\triangle \mathrm{Nuc} / .}$. No significant differences were observed in expression levels of all other

942 domains. g. Western blot analysis of ERCC1 in whole cell extract (WCE). *: non-specific band.

$943 \beta$-tubulin: Loading control. h. Western blot analysis of ERCC1 in chromatin fraction of $X p f^{+/-}$

944 and $X p f^{\triangle \mathrm{Nuc} /-}$ clones. H3: Histone H3 loading control. Note presence of ERCC1 signal in in-

945 frame deleted $X p f^{\Delta \mathrm{Nuc} /-}$ clone \#1, and absence of ERCC1 signal in frame-shifted $X p f^{\Delta \mathrm{Nuc} /}$ clones

$946 \# 2,11$ and 13 i. Cell cycle distribution of $X p f^{+/-}$clone $\# 6$ and $X p f^{A \mathrm{Nuc} /-}$ clone $\# 1$, either untreated

947 or treated with $20,30,40$ or $50 \mathrm{ng} / \mathrm{mL}$ of MMC. Data shows mean values, $\mathrm{n}=3$. Error bars: s.e.m.

948 Untreated samples were compared using the Student's $t$-test; MMC-treated groups were

949 compared by one-way ANOVA. $\mathbf{j}$ and $\mathbf{k}$. Tus/Ter-induced LTGC (j) and I-SceI induced LTGC

950 (k) in $X p f^{f^{+-}}$and $X p f^{A \mathrm{Nuc} /-}$ clones. Analysis by ANOVA, $\mathrm{n}=6$. Error bars: s.e.m.

951

952 
954 1. Berti, M., Cortez, D. \& Lopes, M. The plasticity of DNA replication forks in response to 955 clinically relevant genotoxic stress. Nat Rev Mol Cell Biol 21, 633-651 (2020).

956 2. Cortez, D. Replication-Coupled DNA Repair. Mol Cell 74, 866-876 (2019).

957 3. Quinet, A., Tirman, S., Cybulla, E., Meroni, A. \& Vindigni, A. To skip or not to skip: $958 \quad$ choosing repriming to tolerate DNA damage. Mol Cell 81, 649-658 (2021).

959 4. Crossley, M.P., Bocek, M. \& Cimprich, K.A. R-Loops as Cellular Regulators and $960 \quad$ Genomic Threats. Mol Cell 73, 398-411 (2019).

961 5. Semlow, D.R., Zhang, J., Budzowska, M., Drohat, A.C. \& Walter, J.C. Replication962 Dependent Unhooking of DNA Interstrand Cross-Links by the NEIL3 Glycosylase. Cell $963 \quad \mathbf{1 6 7}, 498-511$ e14 (2016).

964 6. Hodskinson, M.R. et al. Alcohol-derived DNA crosslinks are repaired by two distinct 965 mechanisms. Nature 579, 603-608 (2020).

966 7. Huang, J. et al. The DNA translocase FANCM/MHF promotes replication traverse of 967 DNA interstrand crosslinks. Mol Cell 52, 434-46 (2013).

968 8. Semlow, D.R. \& Walter, J.C. Mechanisms of Vertebrate DNA Interstrand Cross-Link 969 Repair. Annu Rev Biochem (2021).

9709 9. Niraj, J., Färkkilä, A. \& D'Andrea, A.D. The Fanconi Anemia Pathway in Cancer. Annu $971 \quad$ Rev Cancer Biol 3, 457-478 (2019).

972 10. Taylor, A.M.R. et al. Chromosome instability syndromes. Nat Rev Dis Primers 5, 64 973 (2019). 
974 11. Garaycoechea, J.I. et al. Alcohol and endogenous aldehydes damage chromosomes and 975 mutate stem cells. Nature 553, 171-177 (2018).

976 12. Langevin, F., Crossan, G.P., Rosado, I.V., Arends, M.J. \& Patel, K.J. Fancd2 counteracts 977 the toxic effects of naturally produced aldehydes in mice. Nature 475, 53-8 (2011).

978 13. Rosado, I.V., Langevin, F., Crossan, G.P., Takata, M. \& Patel, K.J. Formaldehyde 979 catabolism is essential in cells deficient for the Fanconi anemia DNA-repair pathway. Nat $980 \quad$ Struct Mol Biol 18, 1432-4 (2011).

981 14. O'Brien, P.J., Siraki, A.G. \& Shangari, N. Aldehyde sources, metabolism, molecular 982 toxicity mechanisms, and possible effects on human health. Crit Rev Toxicol 35, 609-62 $983 \quad$ (2005).

984 15. Raschle, M. et al. Mechanism of replication-coupled DNA interstrand crosslink repair. $985 \quad$ Cell 134, 969-80 (2008).

986 16. Wu, R.A. et al. TRAIP is a master regulator of DNA interstrand crosslink repair. Nature $987 \quad 567,267-272(2019)$.

988 17. Wang, S., Wang, R., Peralta, C., Yaseen, A. \& Pavletich, N.P. Structure of the FA core 989 ubiquitin ligase closing the ID clamp on DNA. Nat Struct Mol Biol 28, 300-309 (2021).

990 18. Shakeel, S. et al. Structure of the Fanconi anaemia monoubiquitin ligase complex. Nature $991 \quad 575,234-237(2019)$.

992 19. Xue, X., Sung, P. \& Zhao, X. Functions and regulation of the multitasking FANCM 993 family of DNA motor proteins. Genes Dev 29, 1777-88 (2015).

994 20. Alcón, P. et al. FANCD2-FANCI is a clamp stabilized on DNA by monoubiquitination of 995 FANCD2 during DNA repair. Nat Struct Mol Biol 27, 240-248 (2020). 
996 21. Tan, W. et al. Monoubiquitination by the human Fanconi anemia core complex clamps 997 FANCI:FANCD2 on DNA in filamentous arrays. Elife $\mathbf{9 ( 2 0 2 0 ) .}$

998 22. Amunugama, R. et al. Replication Fork Reversal during DNA Interstrand Crosslink 999 Repair Requires CMG Unloading. Cell Rep 23, 3419-3428 (2018).

1000 23. Gari, K., Decaillet, C., Delannoy, M., Wu, L. \& Constantinou, A. Remodeling of DNA 1001 replication structures by the branch point translocase FANCM. Proc Natl Acad Sci U S A $1002 \quad \mathbf{1 0 5}, 16107-12(2008)$.

1003 24. Panday, A. et al. FANCM regulates repair pathway choice at stalled replication forks. $1004 \quad$ Mol Cell (2021).

1005 25. Klein Douwel, D. et al. XPF-ERCC1 acts in Unhooking DNA interstrand crosslinks in 1006 cooperation with FANCD2 and FANCP/SLX4. Mol Cell 54, 460-71 (2014).

1007 26. Duxin, J.P. \& Walter, J.C. What is the DNA repair defect underlying Fanconi anemia? 1008 Curr Opin Cell Biol 37, 49-60 (2015).

1009 27. Willis, N.A. et al. BRCA1 controls homologous recombination at Tus/Ter-stalled 1010 mammalian replication forks. Nature 510, 556-9 (2014).

1011 28. Willis, N.A. et al. Mechanism of tandem duplication formation in BRCA1-mutant cells. $1012 \quad$ Nature 551, 590-595 (2017).

1013 29. Lambert, S. et al. Homologous recombination restarts blocked replication forks at the 1014 expense of genome rearrangements by template exchange. Mol Cell 39, 346-59 (2010).

1015 30. Scully, R., Panday, A., Elango, R. \& Willis, N.A. DNA double-strand break repair1016 pathway choice in somatic mammalian cells. Nat Rev Mol Cell Biol 20, 698-714 (2019).

1017 31. Paques, F. \& Haber, J.E. Multiple pathways of recombination induced by double-strand 1018 breaks in Saccharomyces cerevisiae. Microbiol Mol Biol Rev 63, 349-404. (1999). 
1019 32. Wyatt, H.D., Laister, R.C., Martin, S.R., Arrowsmith, C.H. \& West, S.C. The SMX DNA $1020 \quad$ Repair Tri-nuclease. Mol Cell 65, 848-860 e11 (2017).

1021 33. Fekairi, S. et al. Human SLX4 is a Holliday junction resolvase subunit that binds multiple 1022 DNA repair/recombination endonucleases. Cell 138, 78-89 (2009).

1023 34. Svendsen, J.M. et al. Mammalian BTBD12/SLX4 assembles a Holliday junction 1024 resolvase and is required for DNA repair. Cell 138, 63-77 (2009).

1025 35. Saito, T.T., Youds, J.L., Boulton, S.J. \& Colaiácovo, M.P. Caenorhabditis elegans HIM1026 18/SLX-4 interacts with SLX-1 and XPF-1 and maintains genomic integrity in the 1027 germline by processing recombination intermediates. PLoS Genet 5, e1000735 (2009).

1028 36. Andersen, S.L. et al. Drosophila MUS312 and the vertebrate ortholog BTBD12 interact 1029 with DNA structure-specific endonucleases in DNA repair and recombination. Mol Cell $1030 \quad 35,128-35(2009)$.

1031 37. Guervilly, J.H. \& Gaillard, P.H. SLX4: multitasking to maintain genome stability. Crit 1032 Rev Biochem Mol Biol 53, 475-514 (2018).

1033 38. Young, S.J. \& West, S.C. Coordinated roles of SLX4 and MutS $\beta$ in DNA repair and the 1034 maintenance of genome stability. Crit Rev Biochem Mol Biol 56, 157-177 (2021).

1035 39. Kim, Y. et al. Regulation of multiple DNA repair pathways by the Fanconi anemia 1036 protein SLX4. Blood 121, 54-63 (2013).

1037 40. Hodskinson, M.R. et al. Mouse SLX4 is a tumor suppressor that stimulates the activity of 1038 the nuclease XPF-ERCC1 in DNA crosslink repair. Mol Cell 54, 472-84 (2014).

1039 41. Manandhar, M., Boulware, K.S. \& Wood, R.D. The ERCC1 and ERCC4 (XPF) genes $1040 \quad$ and gene products. Gene 569, 153-61 (2015). 
1041 42. Zhang, J. \& Walter, J.C. Mechanism and regulation of incisions during DNA interstrand 1042 cross-link repair. DNA Repair (Amst) 19, 135-42 (2014).

1043 43. Castor, D. et al. Cooperative control of holliday junction resolution and DNA repair by 1044 the SLX1 and MUS81-EME1 nucleases. Mol Cell 52, 221-33 (2013).

1045 44. Ciccia, A., Constantinou, A. \& West, S.C. Identification and characterization of the 1046 human mus81-eme1 endonuclease. J Biol Chem 278, 25172-8 (2003).

1047 45. Ciccia, A., McDonald, N. \& West, S.C. Structural and functional relationships of the 1048 XPF/MUS81 family of proteins. Аппи Rev Biochem 77, 259-87 (2008).

1049 46. Wyatt, H.D., Sarbajna, S., Matos, J. \& West, S.C. Coordinated actions of SLX1-SLX4 1050 and MUS81-EME1 for Holliday junction resolution in human cells. Mol Cell 52, 234-47 $1051 \quad$ (2013).

1052 47. Kim, Y. et al. Mutations of the SLX4 gene in Fanconi anemia. Nat Genet 43, 142-6 $1053 \quad$ (2011).

1054 48. Lachaud, C. et al. Distinct functional roles for the two SLX4 ubiquitin-binding UBZ 1055 domains mutated in Fanconi anemia. J Cell Sci 127, 2811-7 (2014).

1056 49. Stoepker, C. et al. SLX4, a coordinator of structure-specific endonucleases, is mutated in 1057 a new Fanconi anemia subtype. Nat Genet 43, 138-41 (2011).

1058 50. Crossan, G.P. et al. Disruption of mouse Slx4, a regulator of structure-specific nucleases, 1059 phenocopies Fanconi anemia. Nat Genet 43, 147-52 (2011).

1060 51. Yamamoto, K.N. et al. Involvement of SLX4 in interstrand cross-link repair is regulated 1061 by the Fanconi anemia pathway. Proc Natl Acad Sci U S A 108, 6492-6 (2011).

1062 52. Bogliolo, M. et al. Mutations in ERCC4, encoding the DNA-repair endonuclease XPF, 1063 cause Fanconi anemia. Am J Hum Genet 92, 800-6 (2013). 
1064 53. Marín, M. et al. Functional Comparison of XPF Missense Mutations Associated to 1065 Multiple DNA Repair Disorders. Genes (Basel) 10(2019).

1066 54. Bhowmick, R., Minocherhomji, S. \& Hickson, I.D. RAD52 Facilitates Mitotic DNA 1067 Synthesis Following Replication Stress. Mol Cell 64, 1117-1126 (2016).

1068 55. Wu, W. et al. RTEL1 suppresses G-quadruplex-associated R-loops at difficult-to1069 replicate loci in the human genome. Nat Struct Mol Biol 27, 424-437 (2020).

1070 56. Johnson, R.D. \& Jasin, M. Sister chromatid gene conversion is a prominent double-strand 1071 break repair pathway in mammalian cells. Embo $J$ 19, 3398-407 (2000).

1072 57. Chandramouly, G. et al. BRCA1 and CtIP suppress long-tract gene conversion between $1073 \quad$ sister chromatids. Nat Commun 4, 2404 (2013).

1074 58. Nagaraju, G., Odate, S., Xie, A. \& Scully, R. Differential regulation of short- and long1075 1076

1077 59. Puget, N., Knowlton, M. \& Scully, R. Molecular analysis of sister chromatid 1078 recombination in mammalian cells. DNA Repair (Amst) 4, 149-61 (2005).

1079 60. Li, S. et al. PIF1 helicase promotes break-induced replication in mammalian cells. Embo $1080 \quad j, \mathrm{e} 104509(2021)$.

1081 61. Costantino, L. et al. Break-induced replication repair of damaged forks induces genomic 1082 duplications in human cells. Science 343, 88-91 (2014).

1083 62. Dilley, R.L. et al. Break-induced telomere synthesis underlies alternative telomere 1084 maintenance. Nature 539, 54-58 (2016).

1085 63. Huang, J.W. et al. MCM8IP activates the MCM8-9 helicase to promote DNA synthesis 1086 and homologous recombination upon DNA damage. Nat Commun 11, 2948 (2020). 
1087 64. Hustedt, N. et al. Control of homologous recombination by the HROB-MCM8-MCM9 1088 pathway. Genes Dev 33, 1397-1415 (2019).

1089 65. Kosicki, M., Tomberg, K. \& Bradley, A. Repair of double-strand breaks induced by 1090 CRISPR-Cas9 leads to large deletions and complex rearrangements. Nat Biotechnol 36, $1091 \quad 765-771(2018)$.

1092 66. Natsume, T., Kiyomitsu, T., Saga, Y.\& Kanemaki, M.T. Rapid Protein Depletion in 1093 Human Cells by Auxin-Inducible Degron Tagging with Short Homology Donors. Cell $1094 \quad \operatorname{Rep~15,~210-218~(2016).~}$

1095 67. Nishimura, K. et al. A super-sensitive auxin-inducible degron system with an engineered 1096 auxin-TIR1 pair. Nucleic Acids Res (2020).

1097 68. Chung, H.K. et al. Tunable and reversible drug control of protein production via a self1098 excising degron. Nat Chem Biol 11, 713-20 (2015).

1099 69. Wang, X. et al. Role of DNA replication proteins in double-strand break-induced 1100 recombination in Saccharomyces cerevisiae. Mol Cell Biol 24, 6891-9 (2004).

1101 70. Deans, A.J. \& West, S.C. FANCM connects the genome instability disorders Bloom's $1102 \quad$ Syndrome and Fanconi Anemia. Mol Cell 36, 943-53 (2009).

1103 71. Duxin, J.P., Dewar, J.M., Yardimci, H. \& Walter, J.C. Repair of a DNA-protein crosslink 1104 by replication-coupled proteolysis. Cell 159, 346-57 (2014).

1105 72. Larsen, N.B. et al. Replication-Coupled DNA-Protein Crosslink Repair by SPRTN and 1106 the Proteasome in Xenopus Egg Extracts. Mol Cell 73, 574-588.e7 (2019).

1107 73. Sparks, J.L. et al. The CMG Helicase Bypasses DNA-Protein Cross-Links to Facilitate 1108 Their Repair. Cell 176, 167-181 e21 (2019). 
1109 74. Stingele, J., Bellelli, R. \& Boulton, S.J. Mechanisms of DNA-protein crosslink repair.

$1110 \quad$ Nat Rev Mol Cell Biol 18, 563-573 (2017).

1111 75. Wang, R., Wang, S., Dhar, A., Peralta, C. \& Pavletich, N.P. DNA clamp function of the 1112 monoubiquitinated Fanconi anaemia ID complex. Nature 580, 278-282 (2020).

1113 76. Willis, N.A. \& Scully, R. Measurement of Homologous Recombination at Stalled

1114 Mammalian Replication Forks. Methods Mol Biol 2153, 329-353 (2021).

1115 77. Willis, N.A., Panday, A., Duffey, E.E. \& Scully, R. Rad51 recruitment and exclusion of 1116 non-homologous end joining during homologous recombination at a Tus/Ter mammalian 1117 replication fork barrier. PLoS Genet 14, e1007486 (2018).

1118 78. Schmittgen, T.D. \& Livak, K.J. Analyzing real-time PCR data by the comparative C(T) 1119 method. Nat Protoc 3, 1101-8 (2008). 
a

5'Tr-GFP 6xTer-I-Scel-GFP

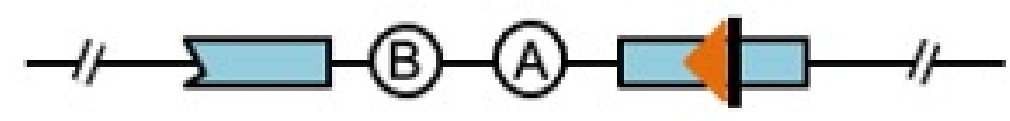

$\downarrow$ Bidirectional fork stalling
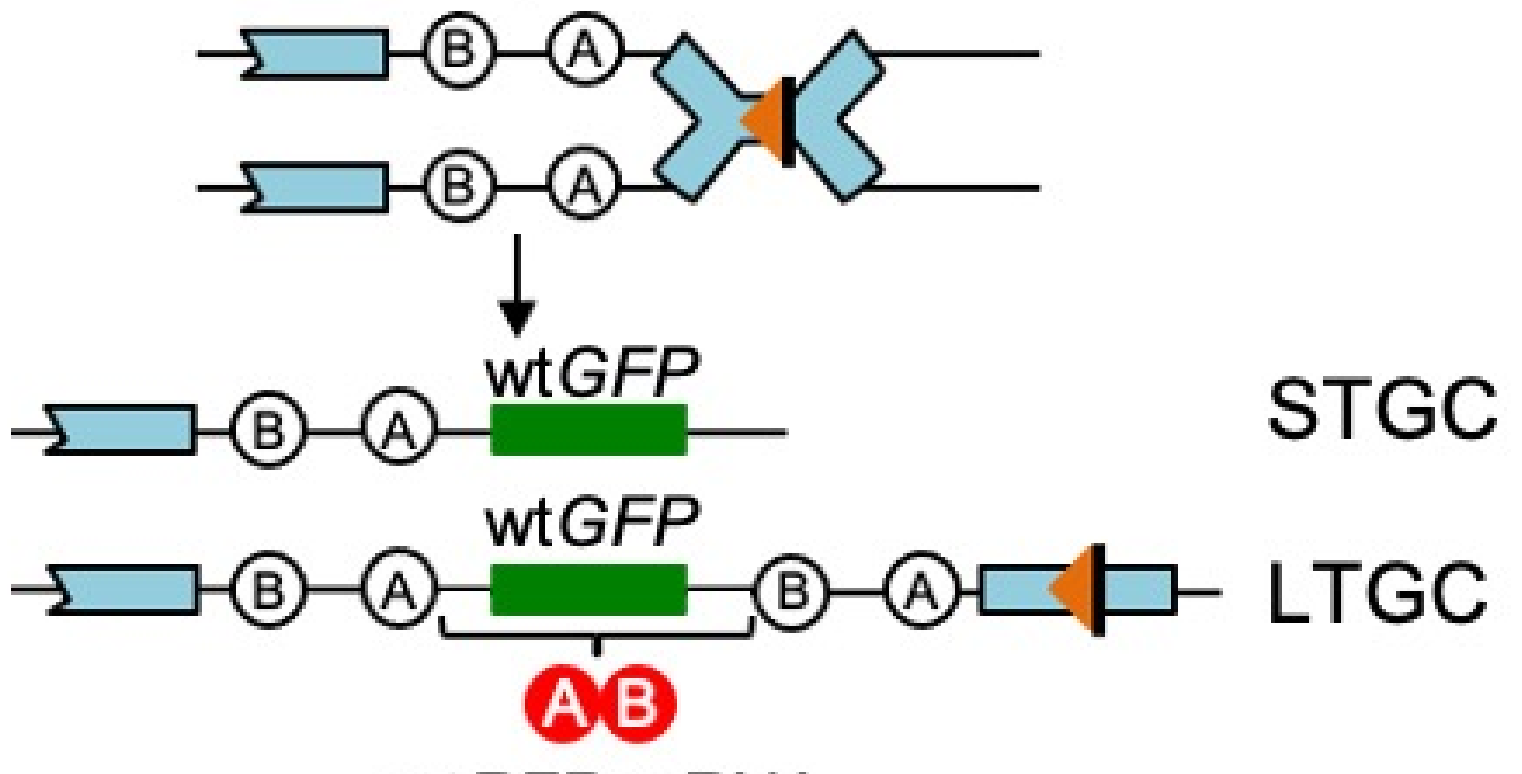

wtRFP mRNA b

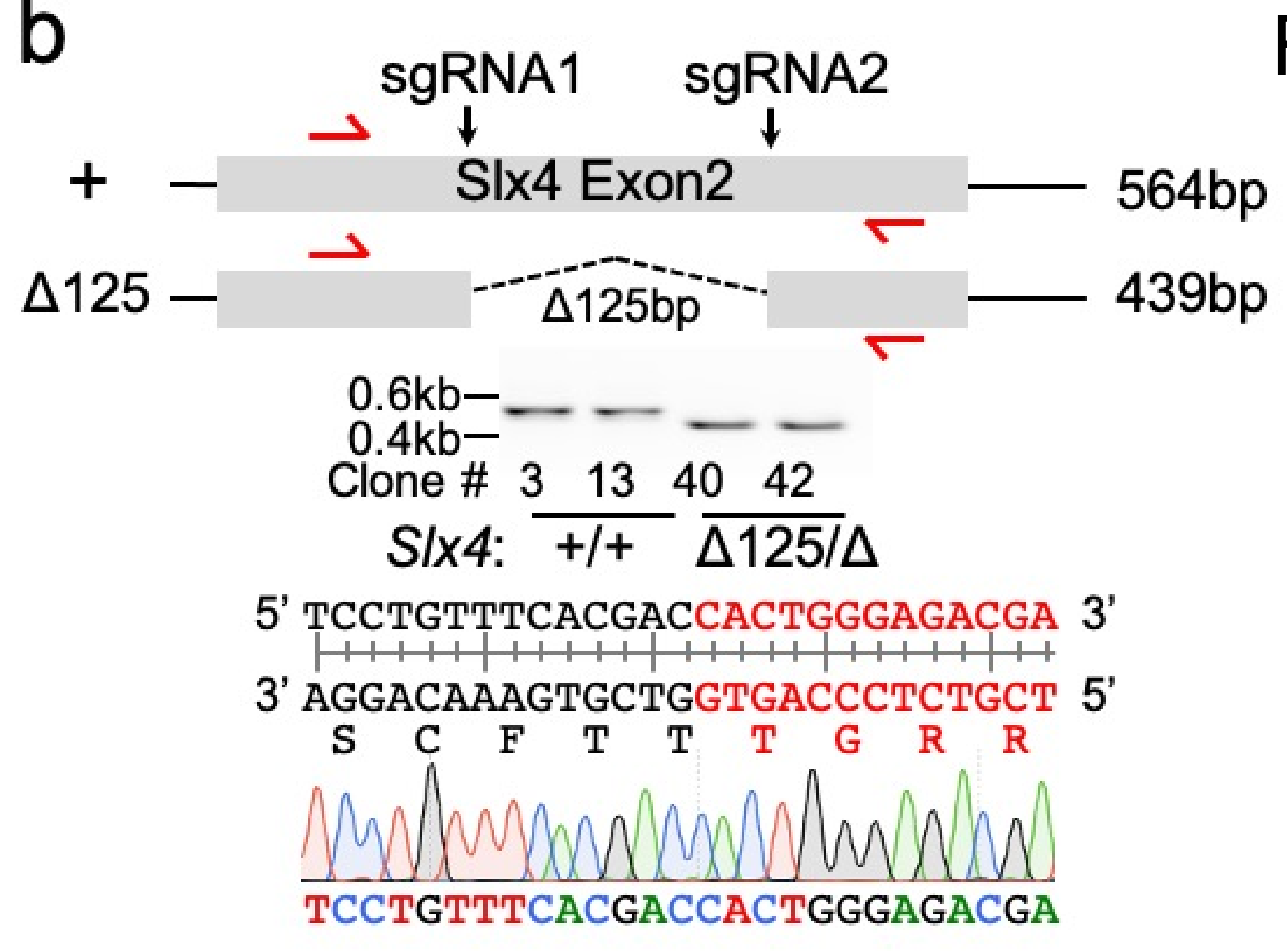

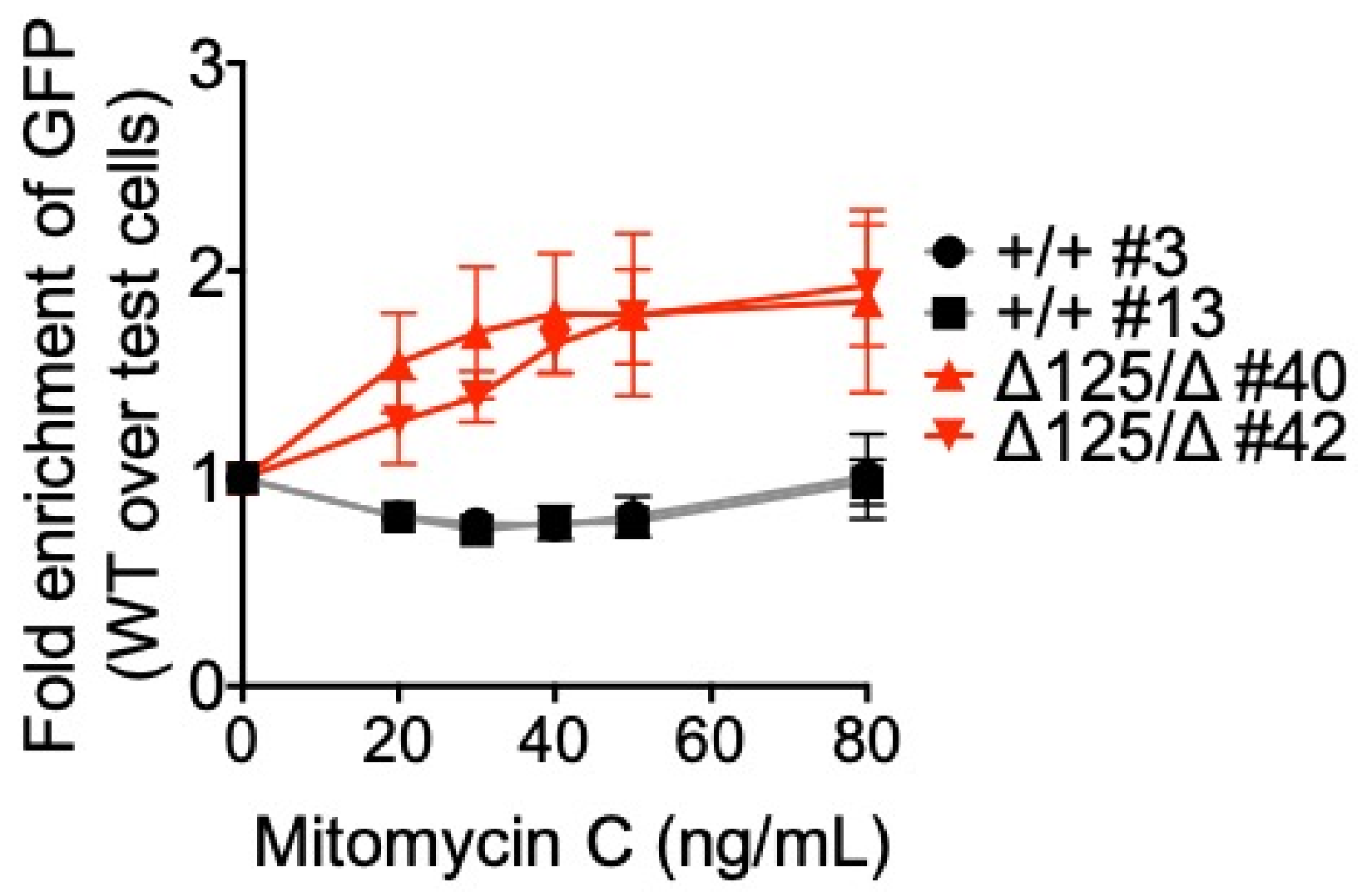

d

S/ $\times 4^{+/+}$Clone \#3

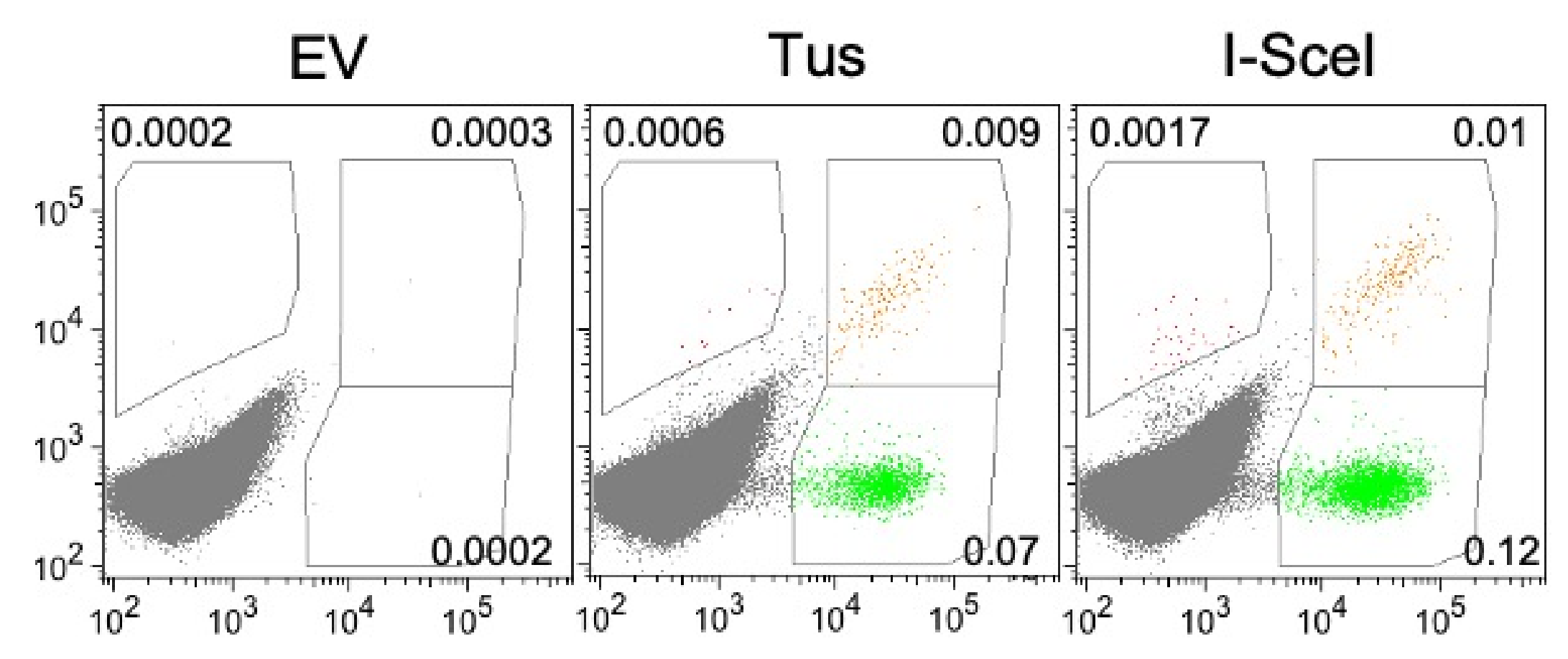

SIX $4^{\Delta 125 / \Delta}$ Clone \#40

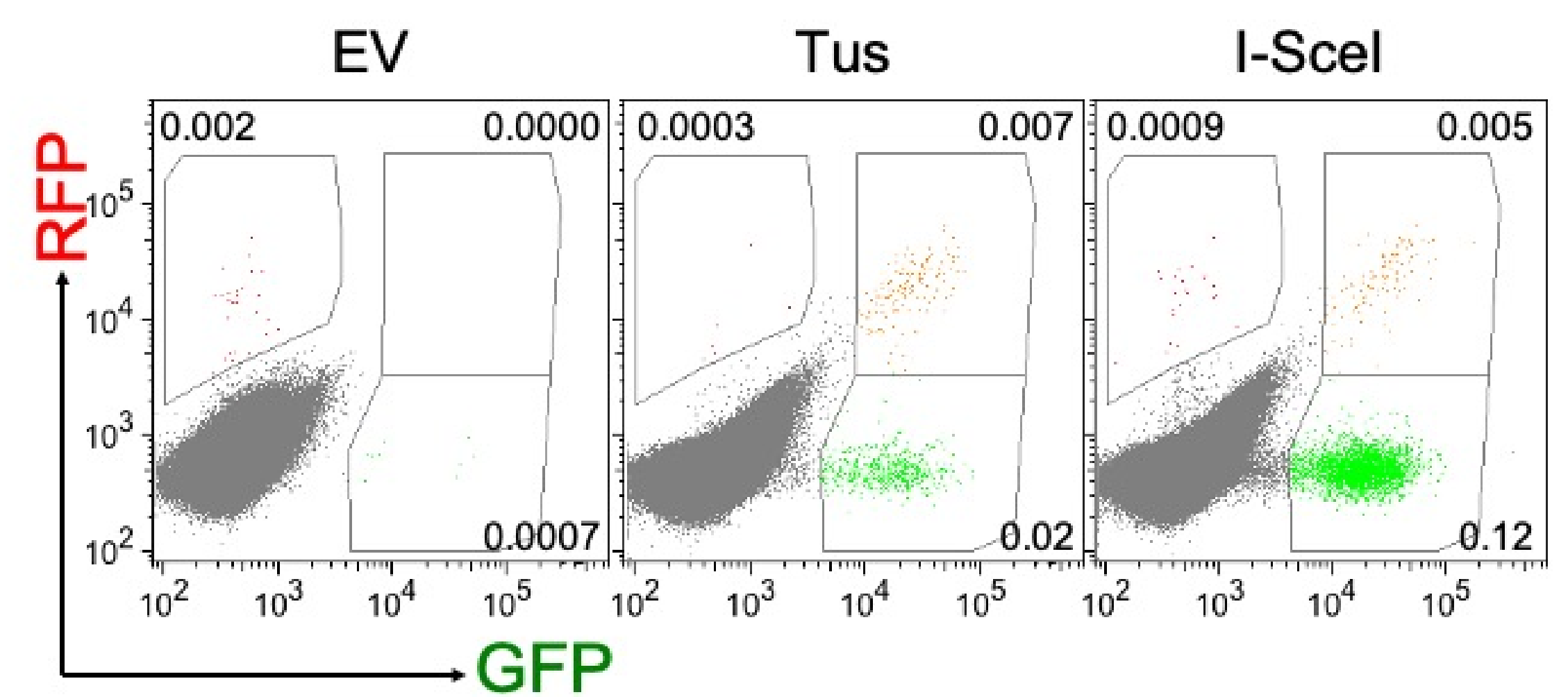

e

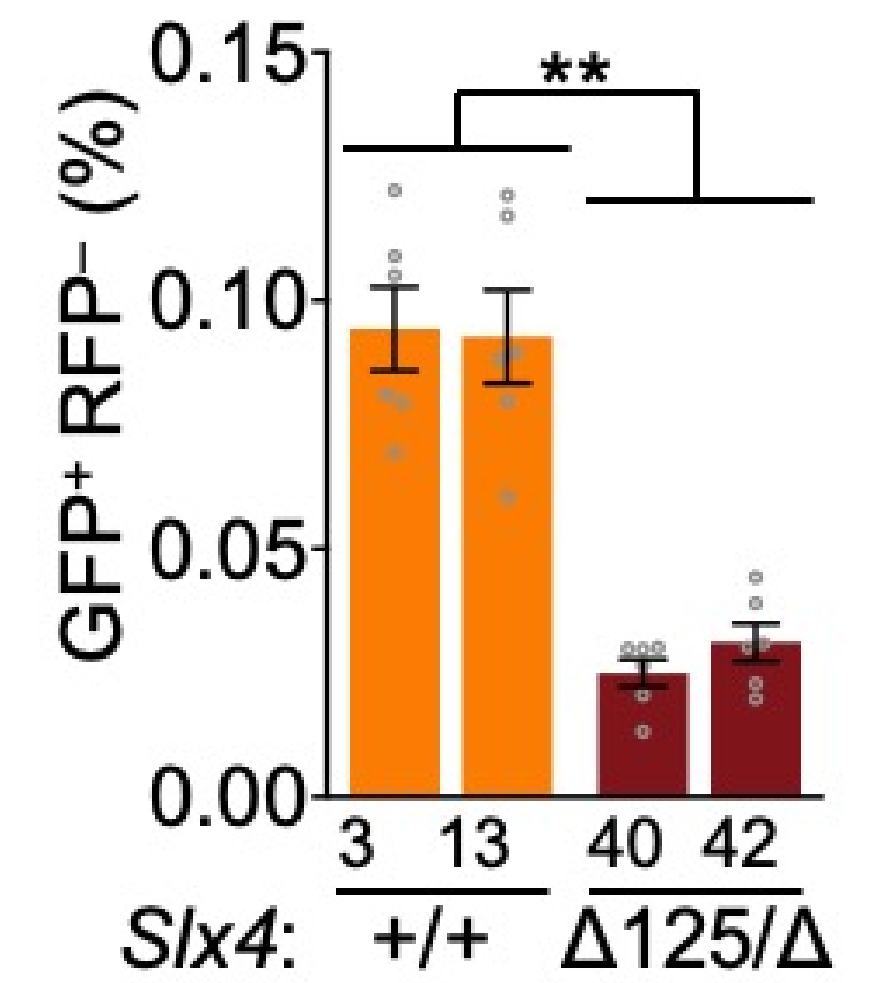

g

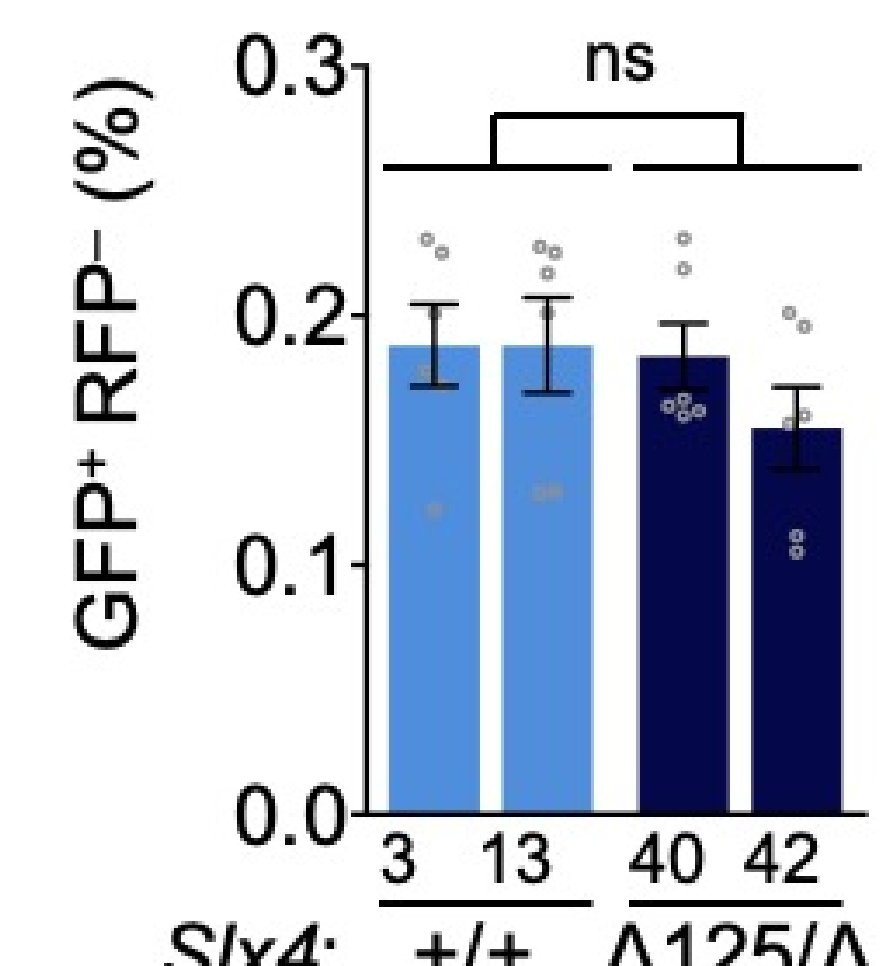

f

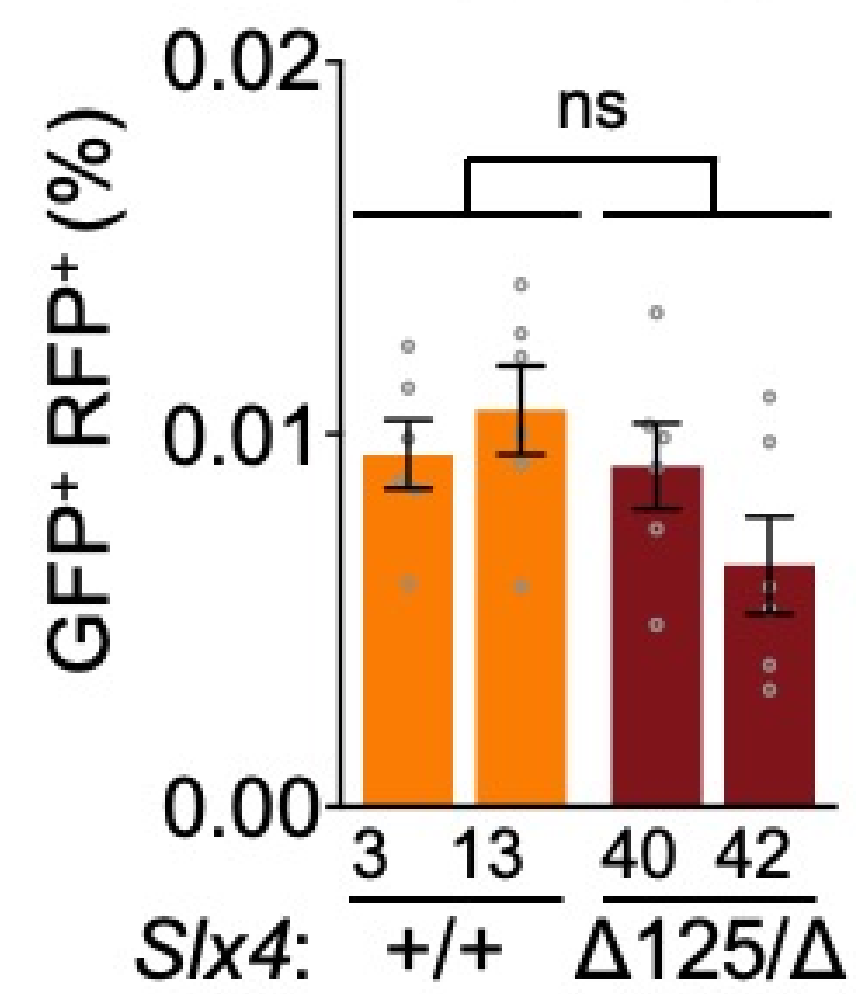

$\mathrm{h}$

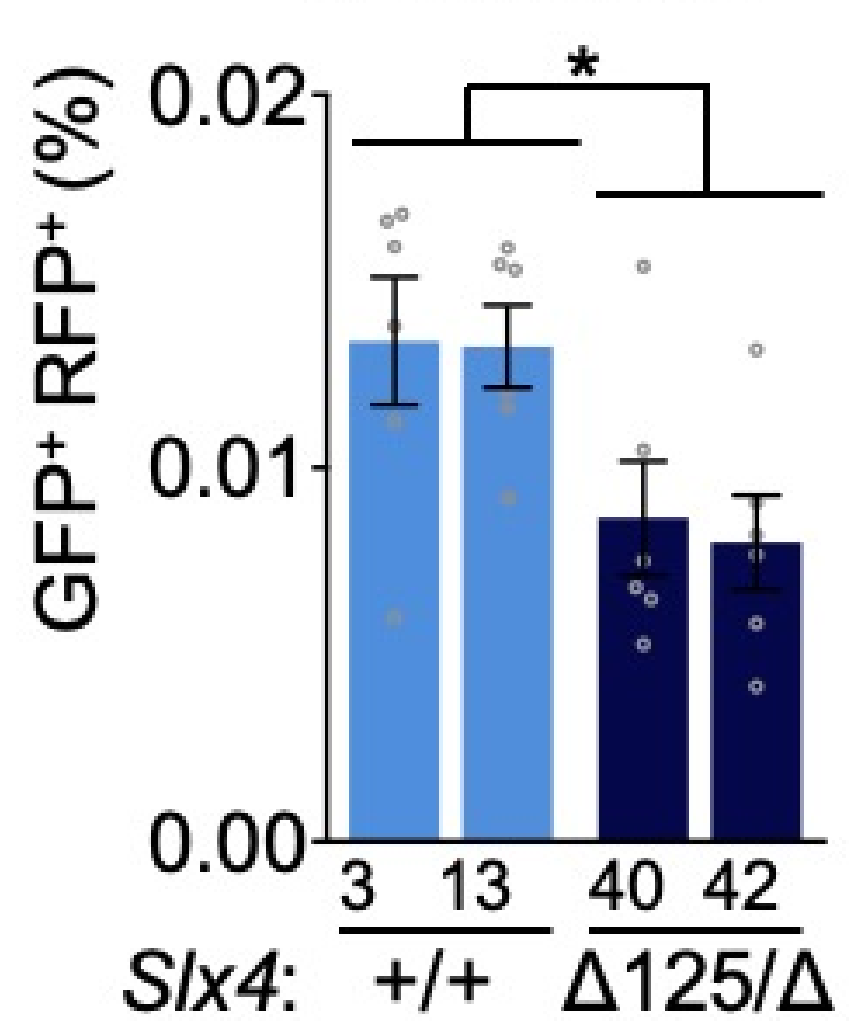


a $\quad$ M.m. SLX4

80185232380454603

$\mathrm{N}$\begin{tabular}{c|c|c} 
UBZ & & \\
\hline $1 / 2$ & MLR & BTB
\end{tabular}

XPF

ERCC1

$\triangle \mathrm{UBZ}$

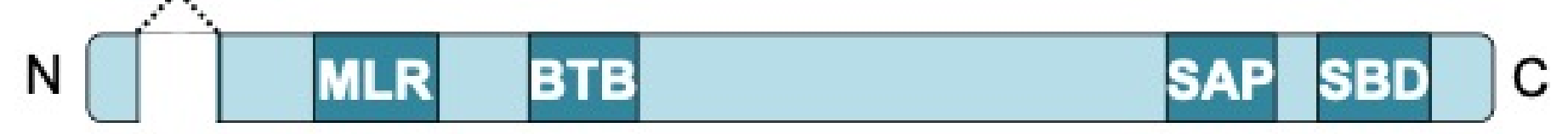

C

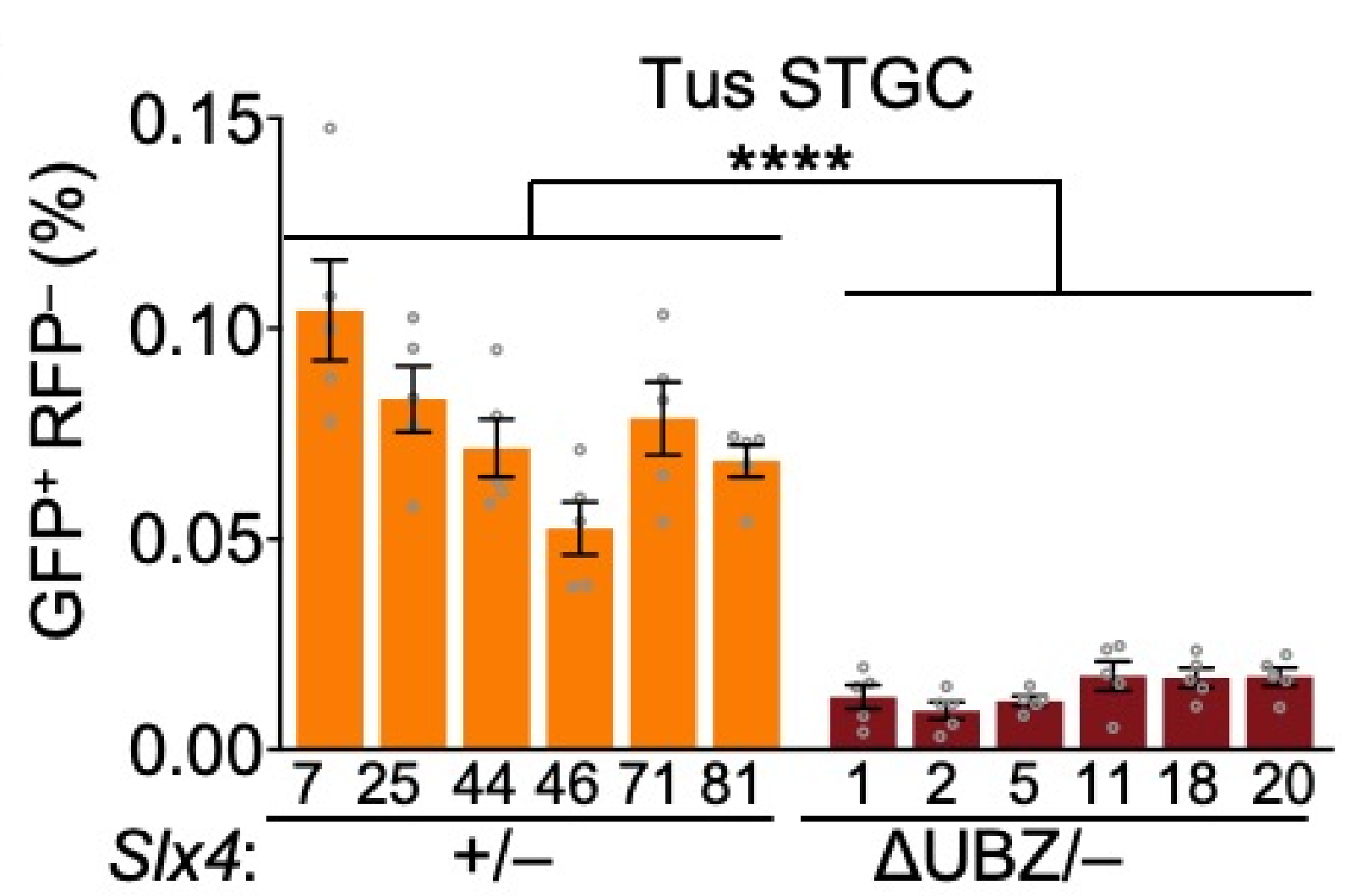

e

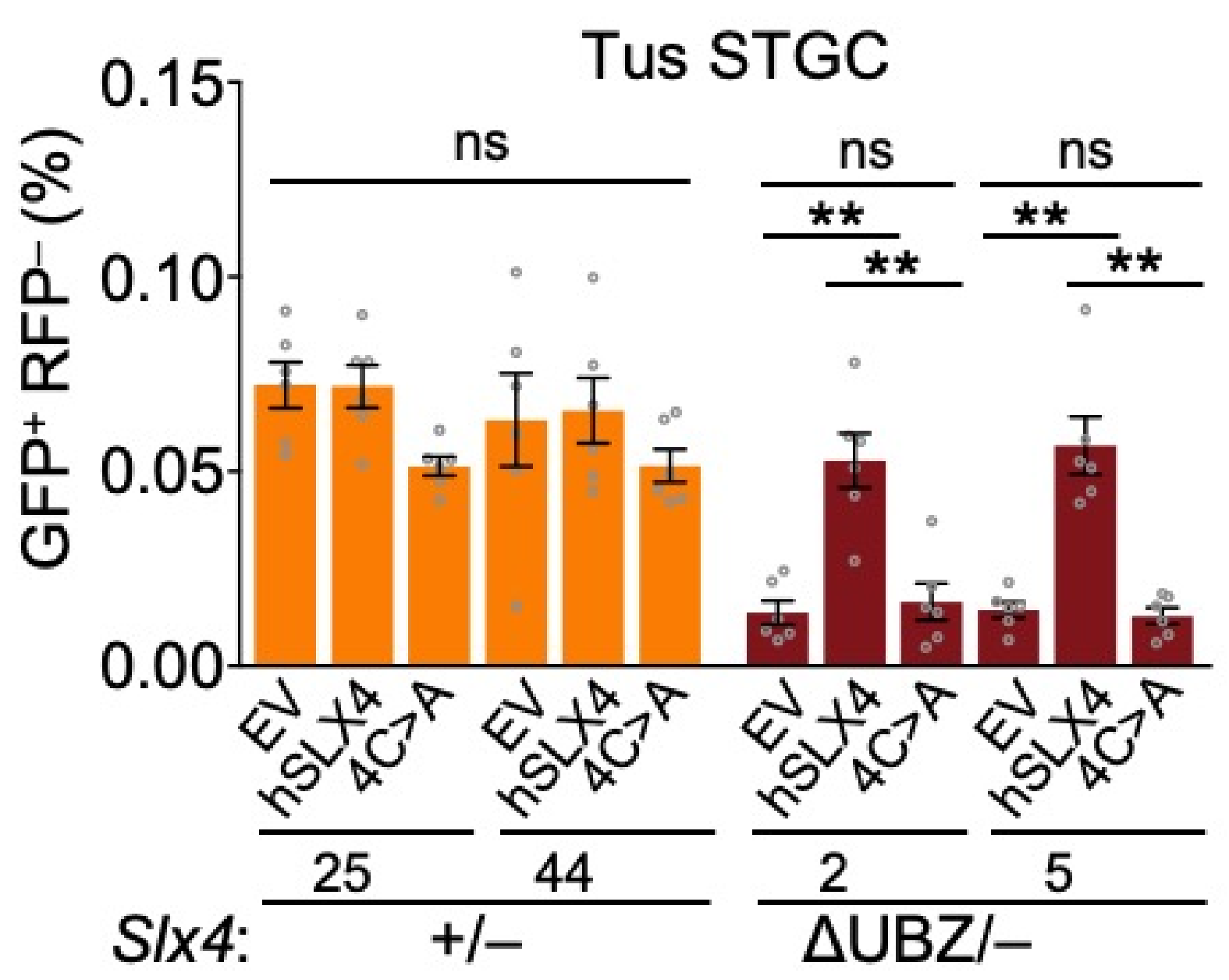

g

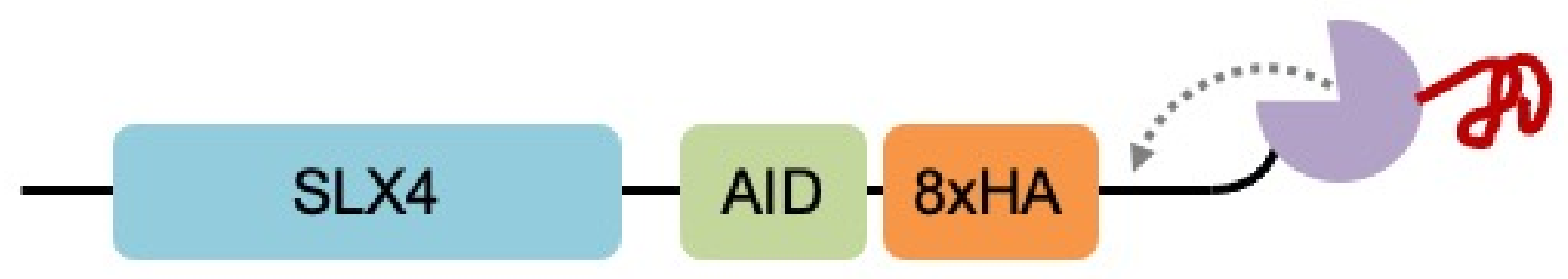

h ${ }_{S / \times 4 \text { RTAPCR }} \mathrm{i}$

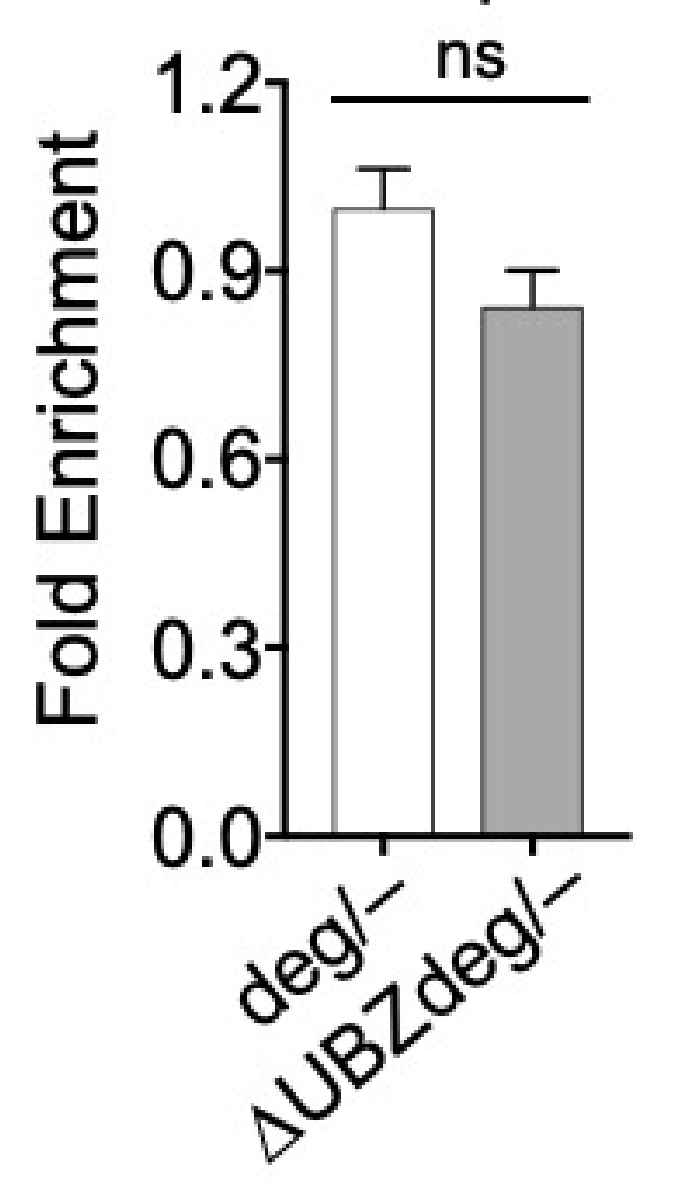

$\mathrm{kDa} \quad \mathrm{deg} /-\quad \Delta \mathrm{UBZ}$ deg/-
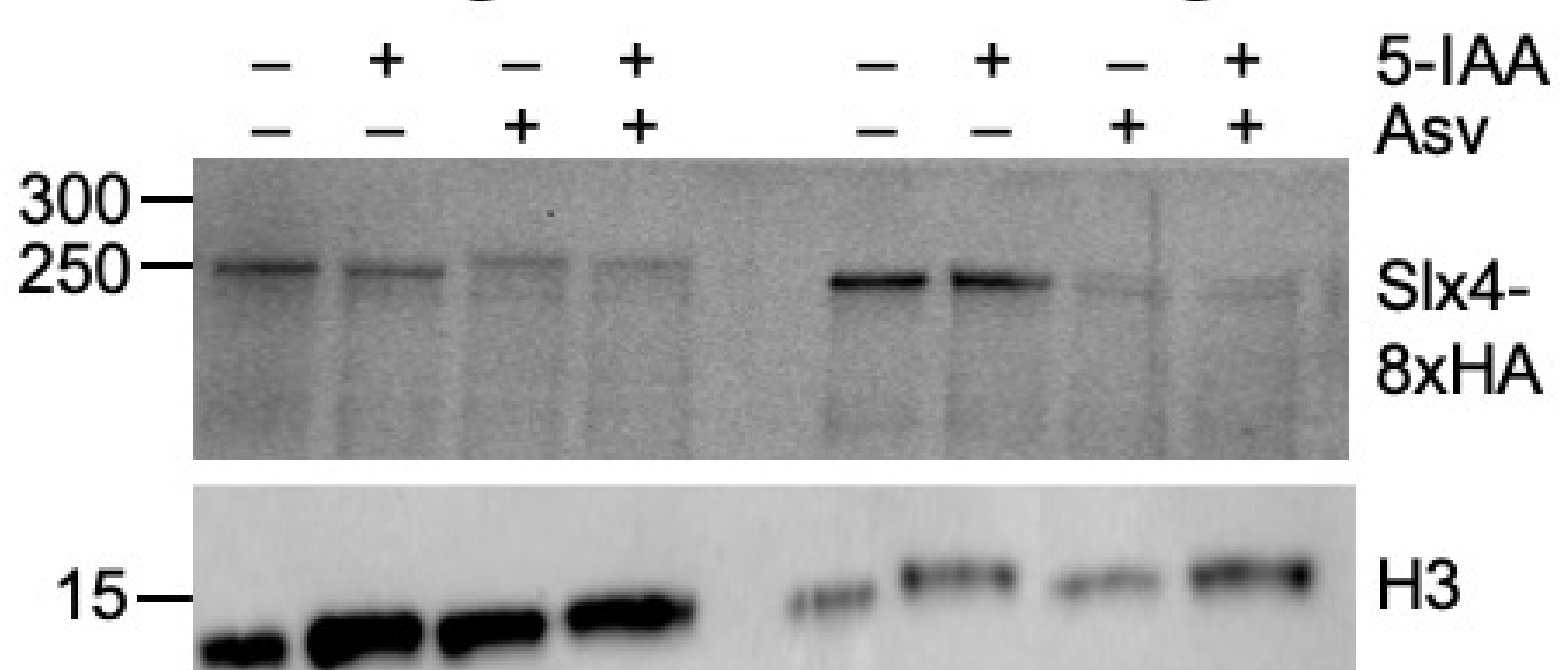

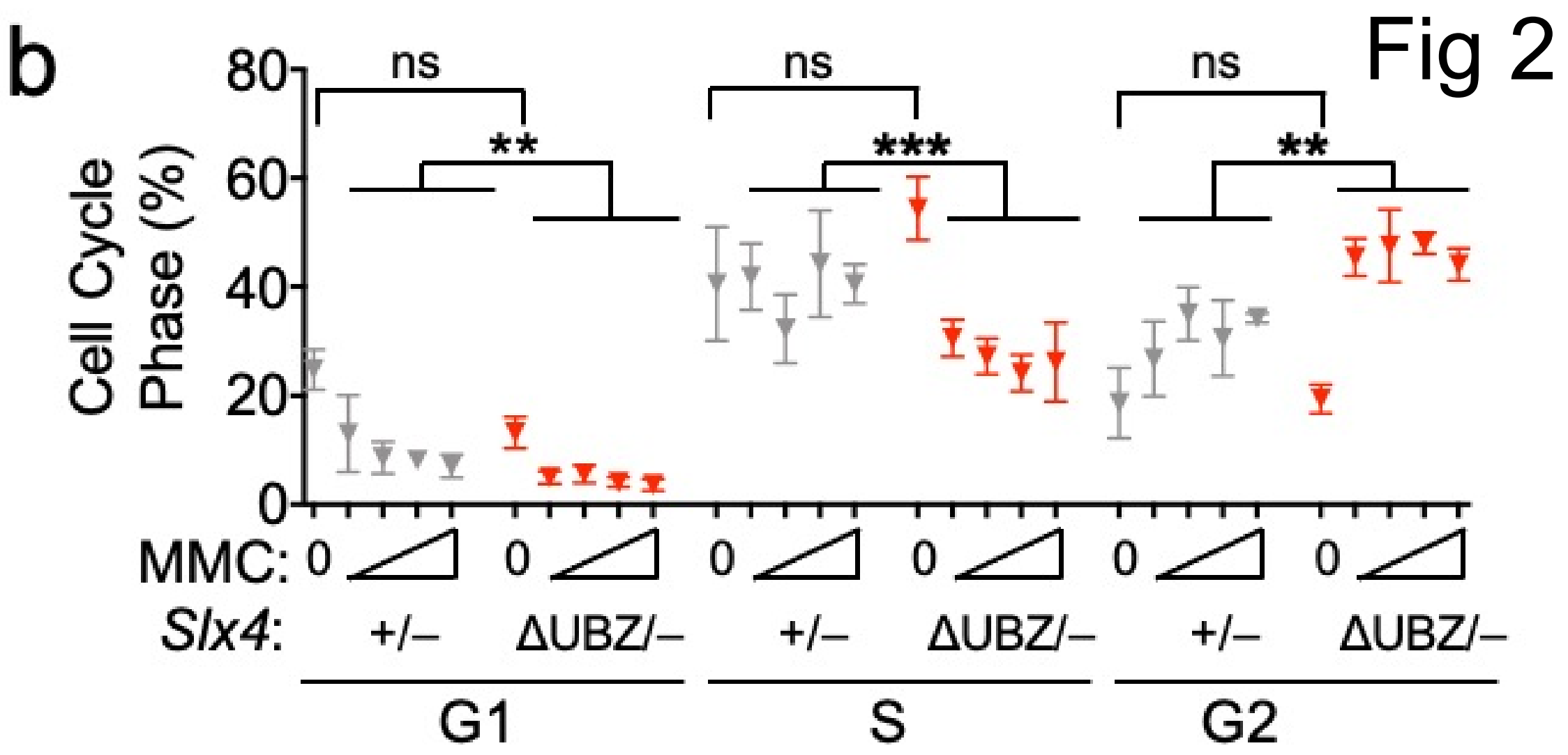

d
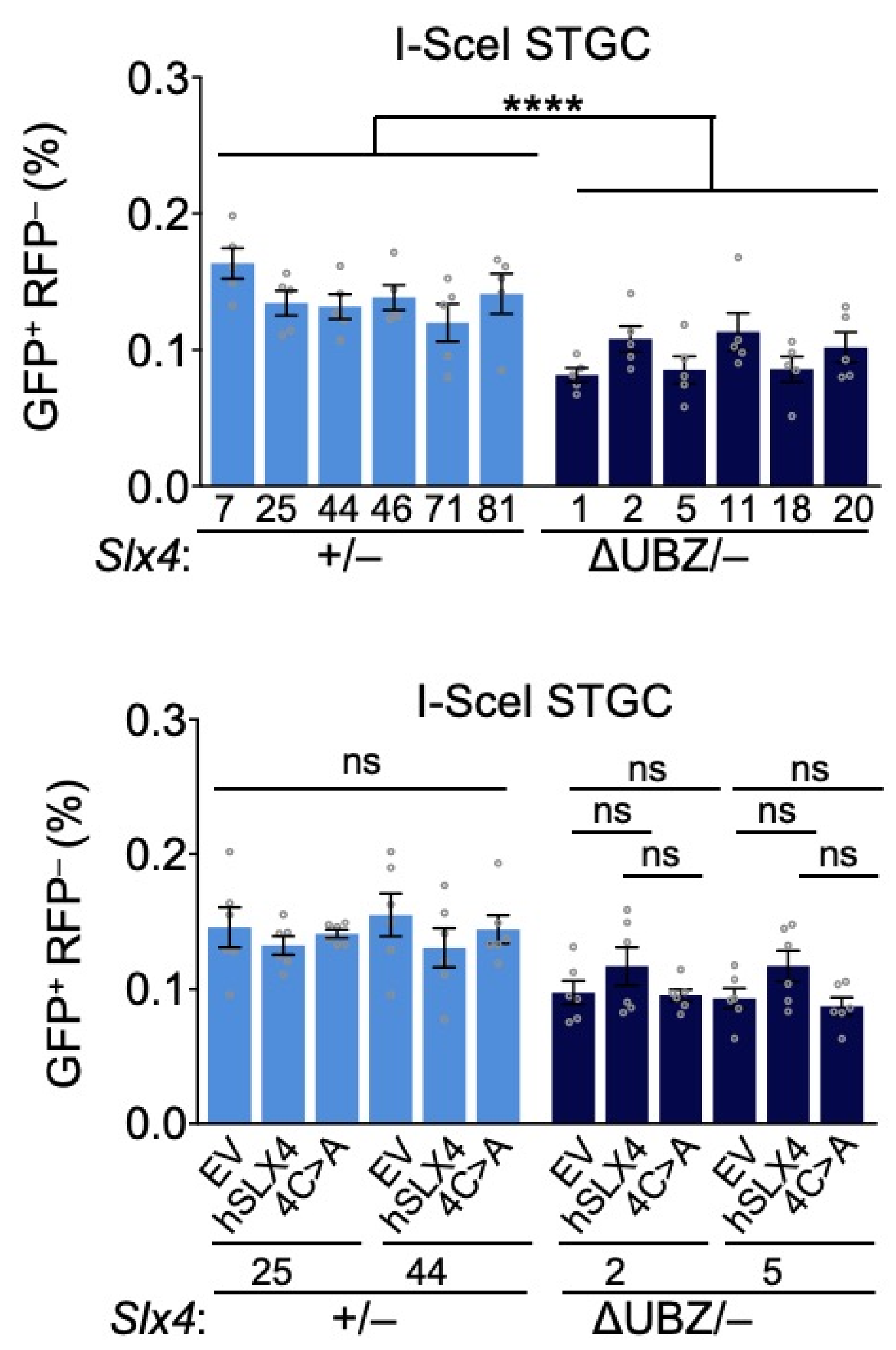

j

\section{SLX4-HA ChIP}

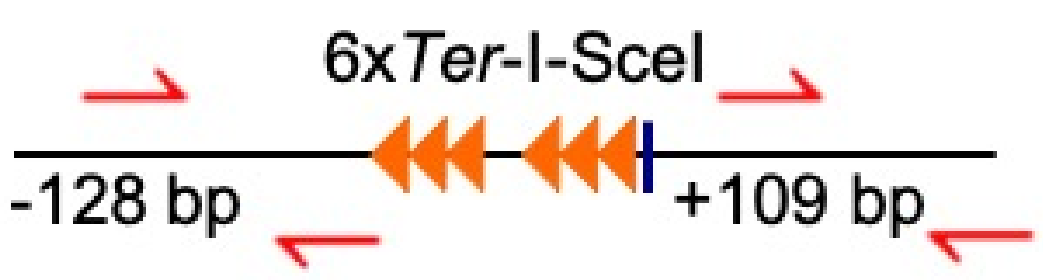

EV

$\frac{n s}{10}$

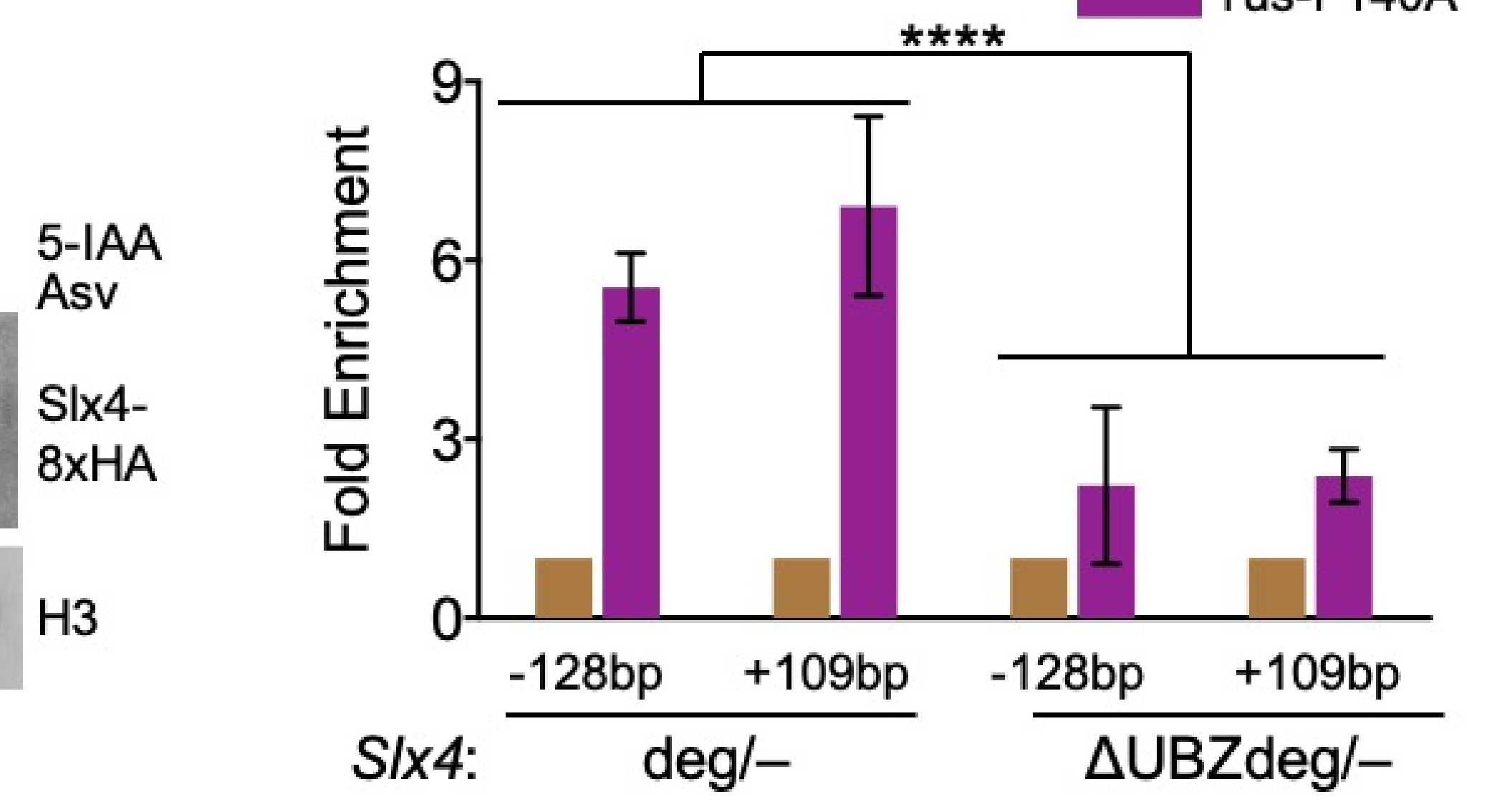


$b$
$d$

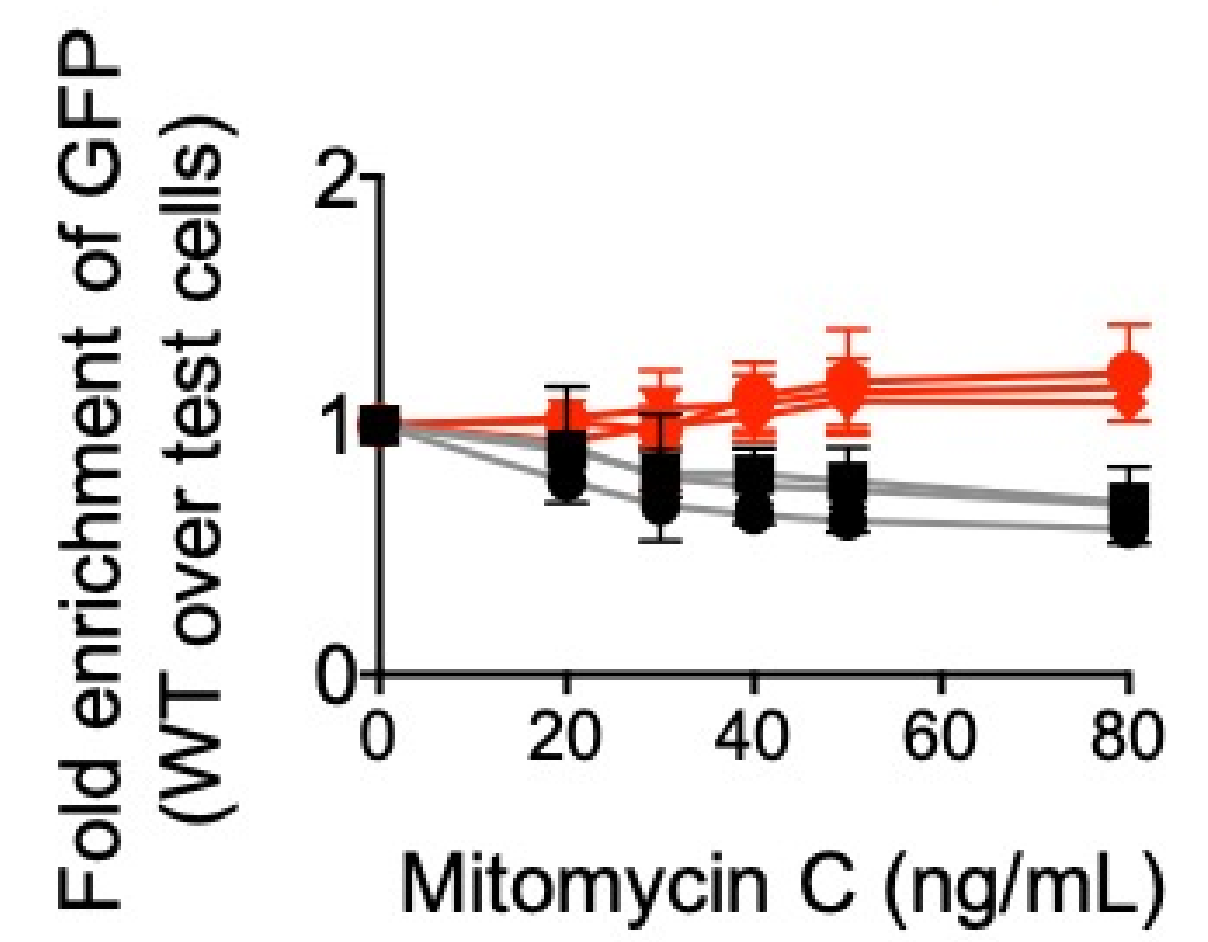

- +l-\#5

- + - \#8

¿ +l-\#17

* $\triangle \mathrm{SAP} / \mathrm{S}$ - 21

- $\triangle$ SAPI-\#28

- $\triangle \mathrm{SAP} / \mathrm{Z}$ \#1

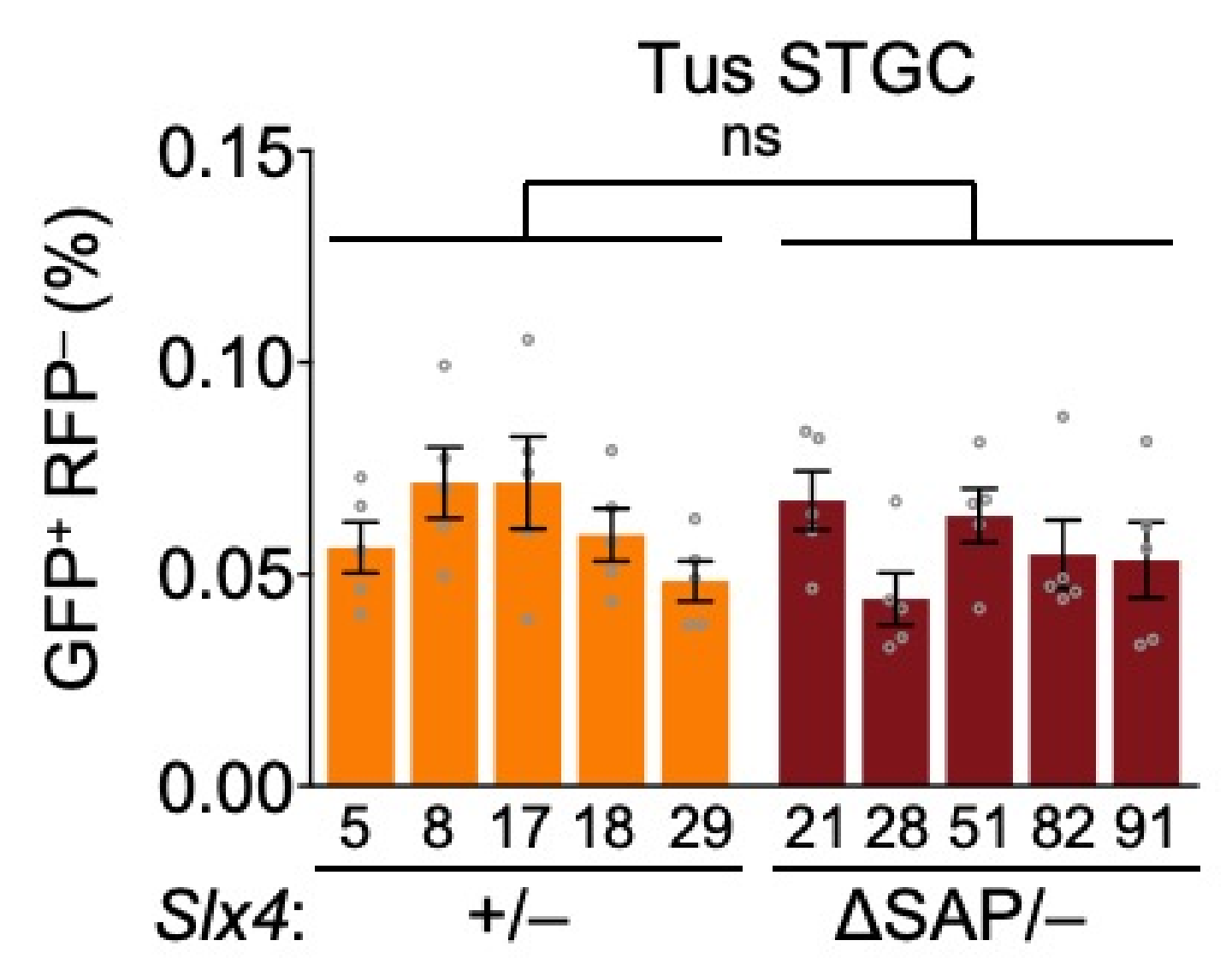

e

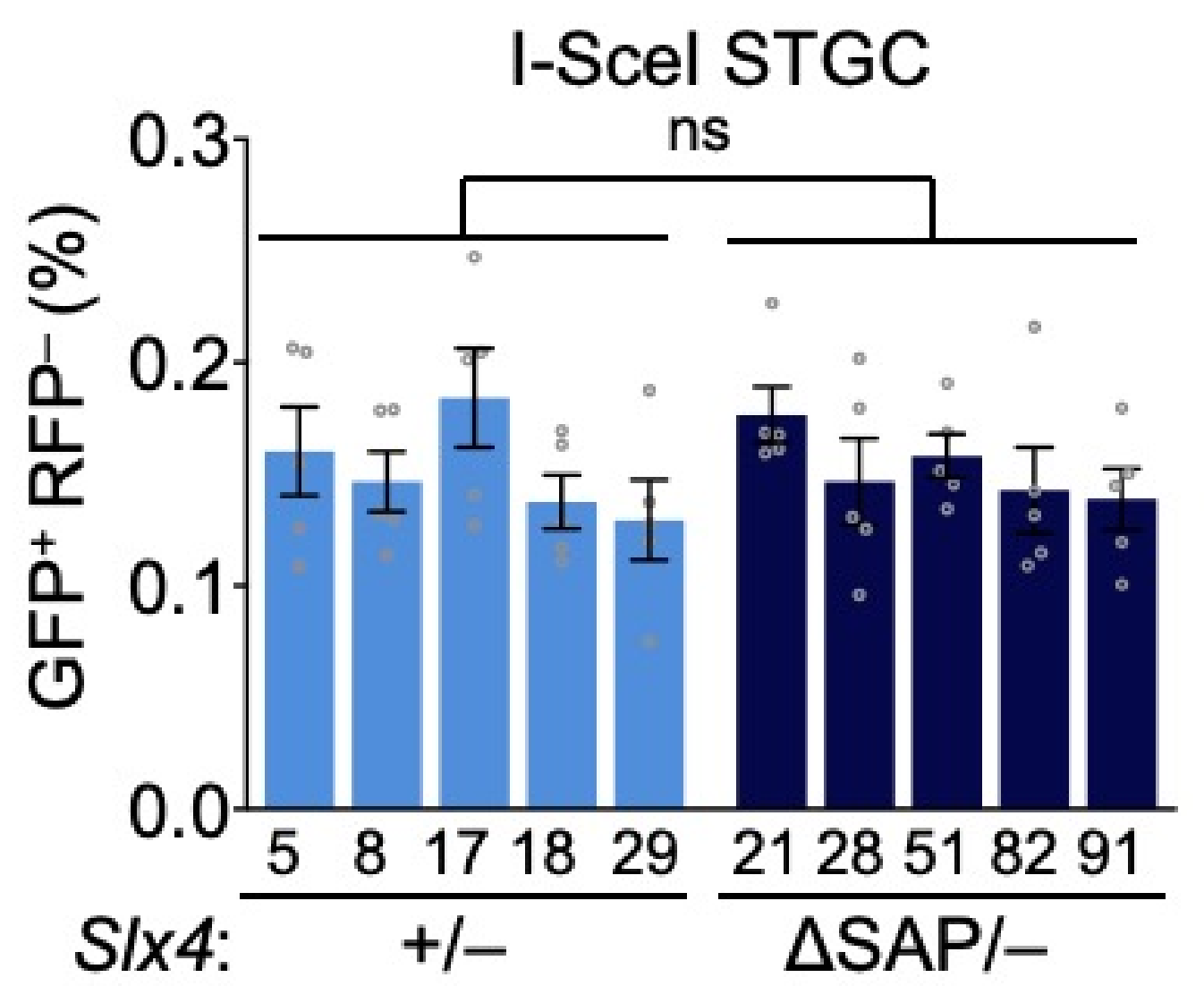

f

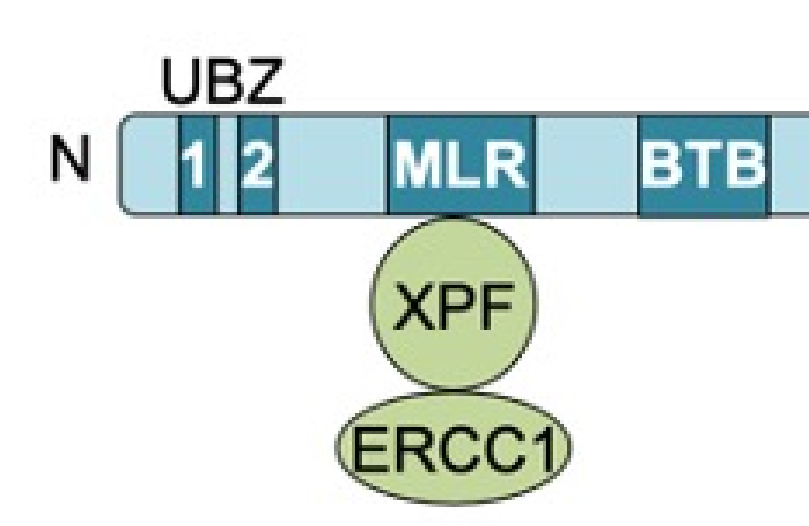

요

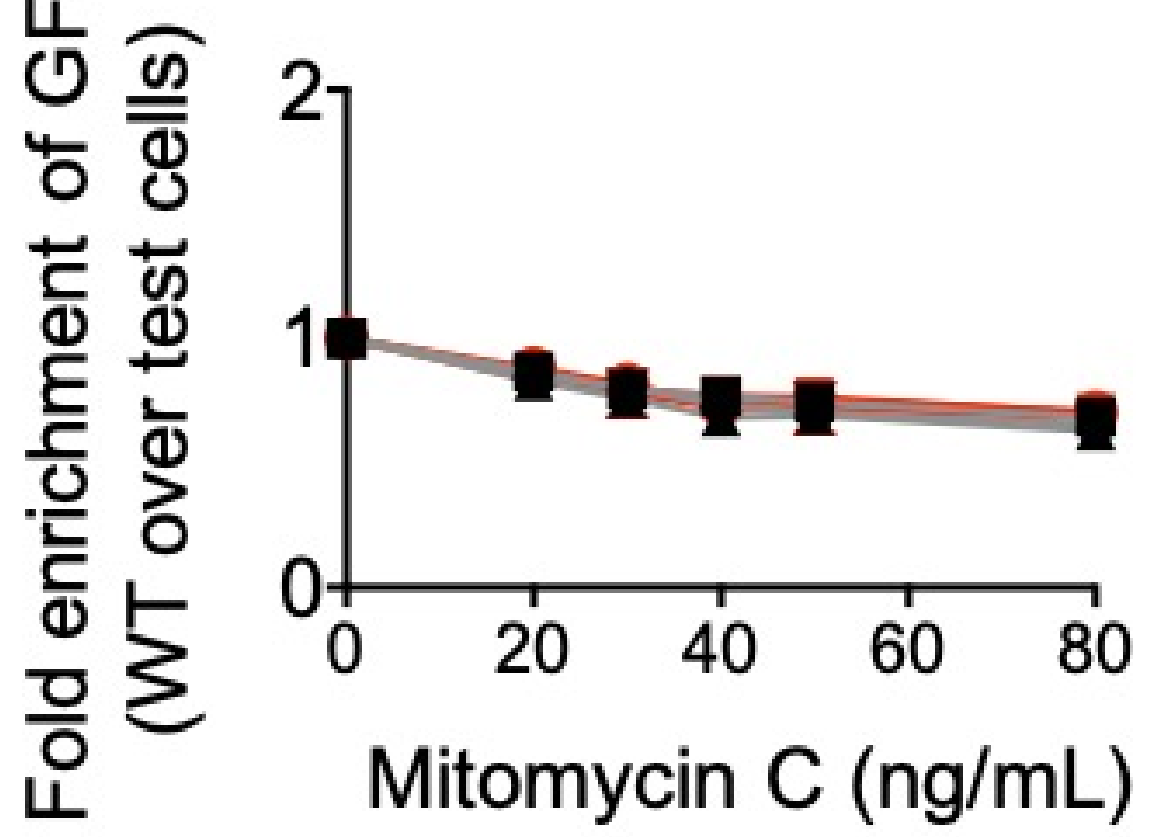

$+/-\# 2$

$\pm+1-\# 22$

* $\triangle \mathrm{SBD} /-\# 15$

- $\triangle \mathrm{SBD} /-\# 29$

- $\triangle \mathrm{SBD} /-\# 31$

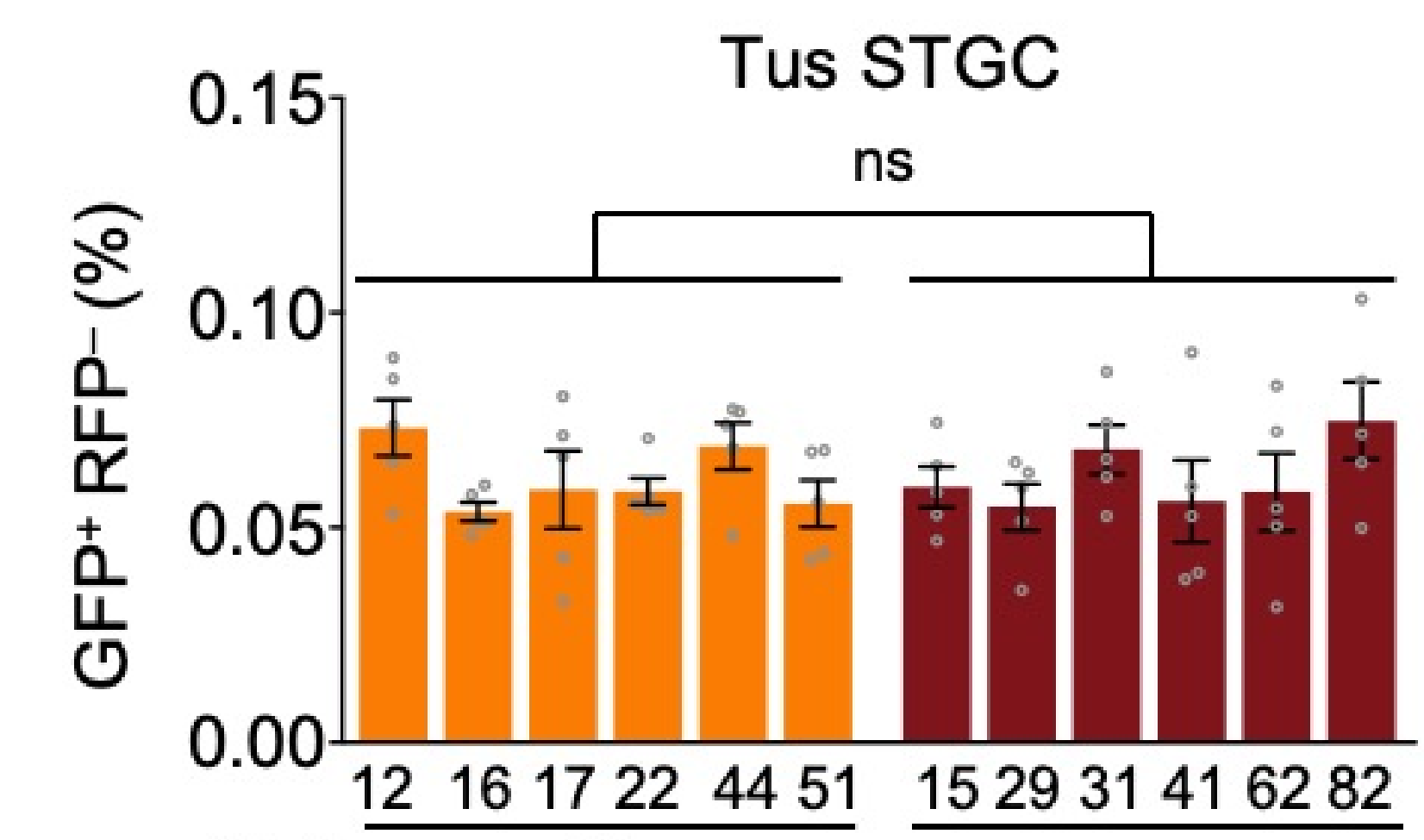

S/X4:

$\triangle \mathrm{SBD} /-$

h

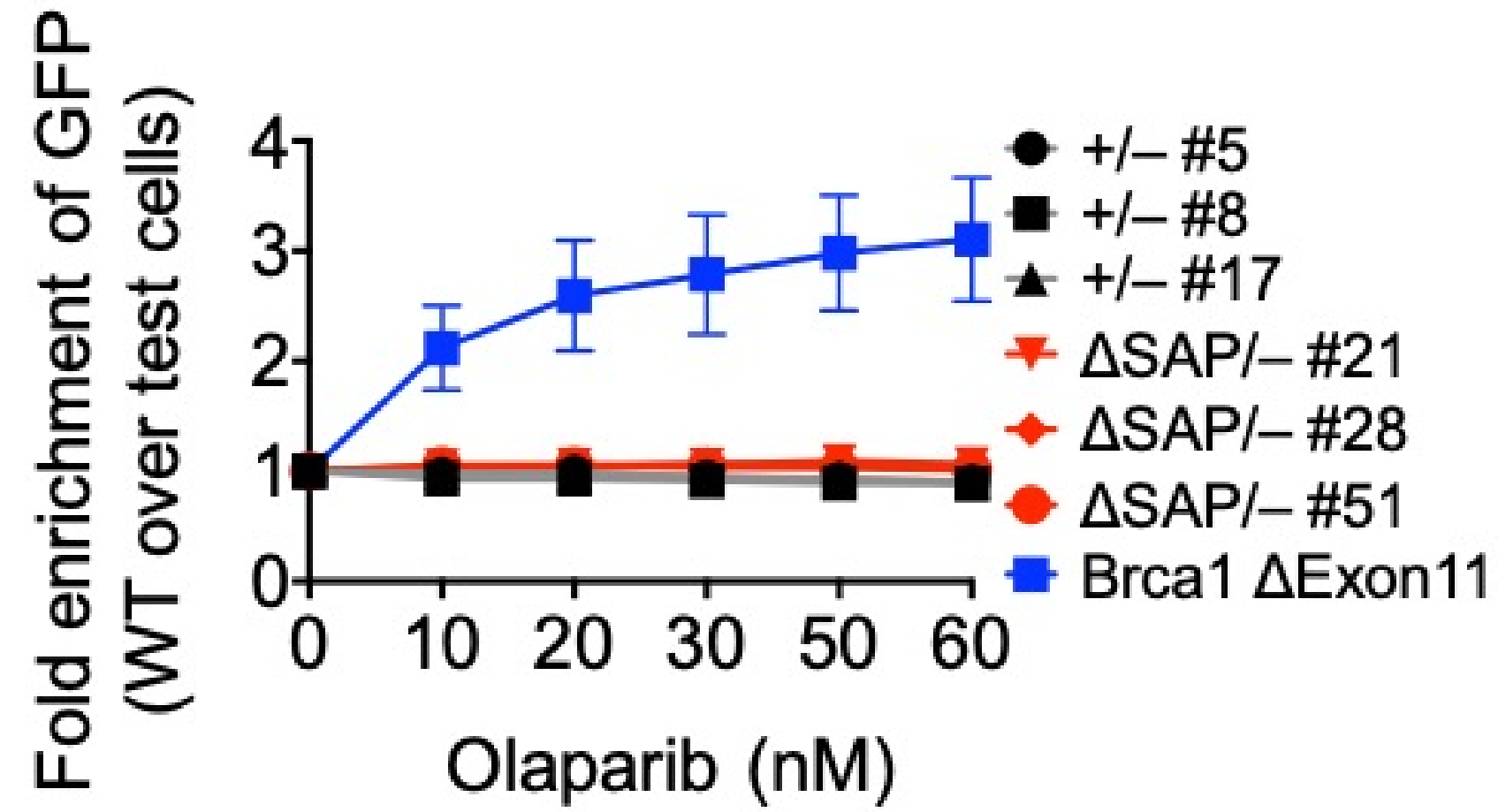

$\triangle S B D$

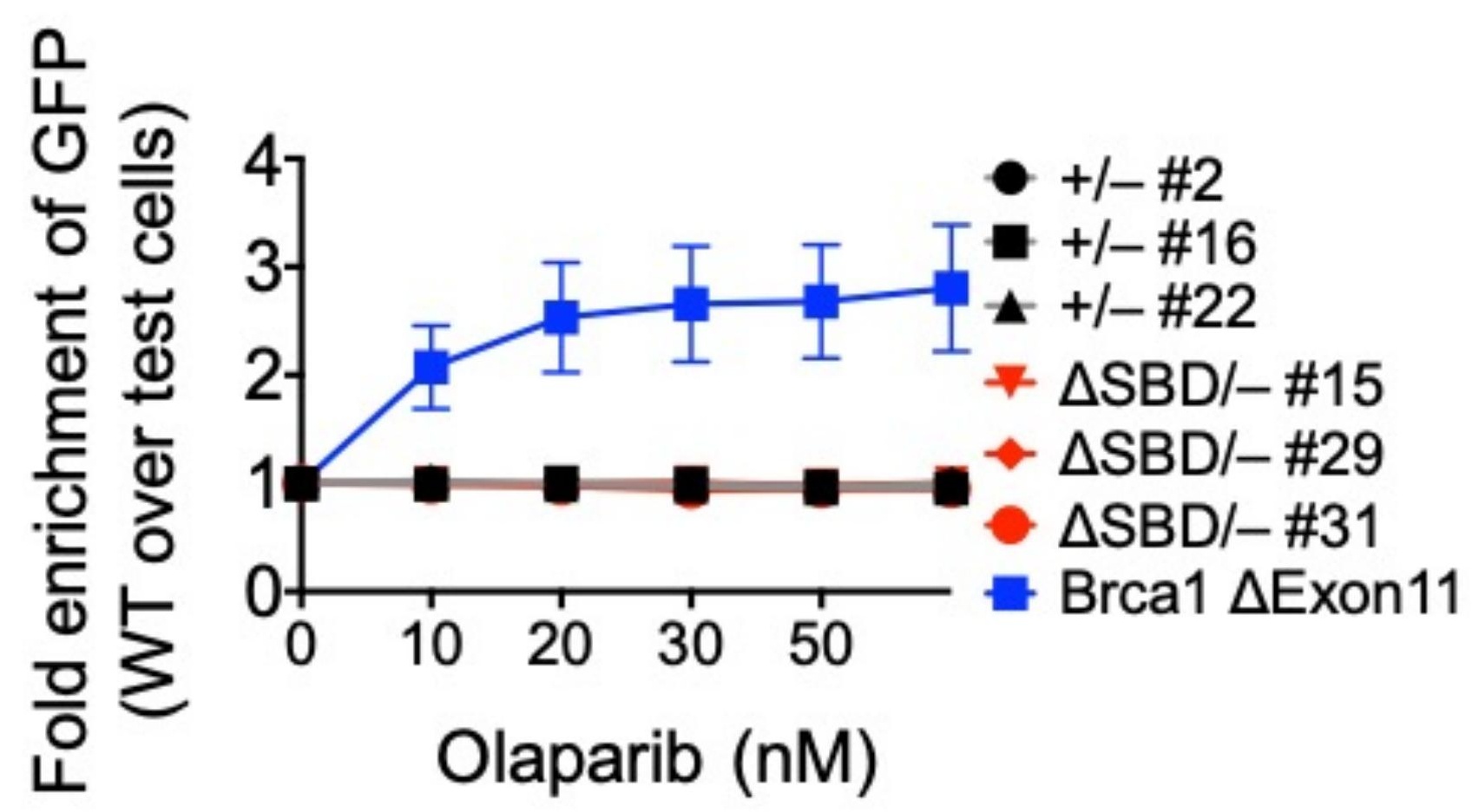

j

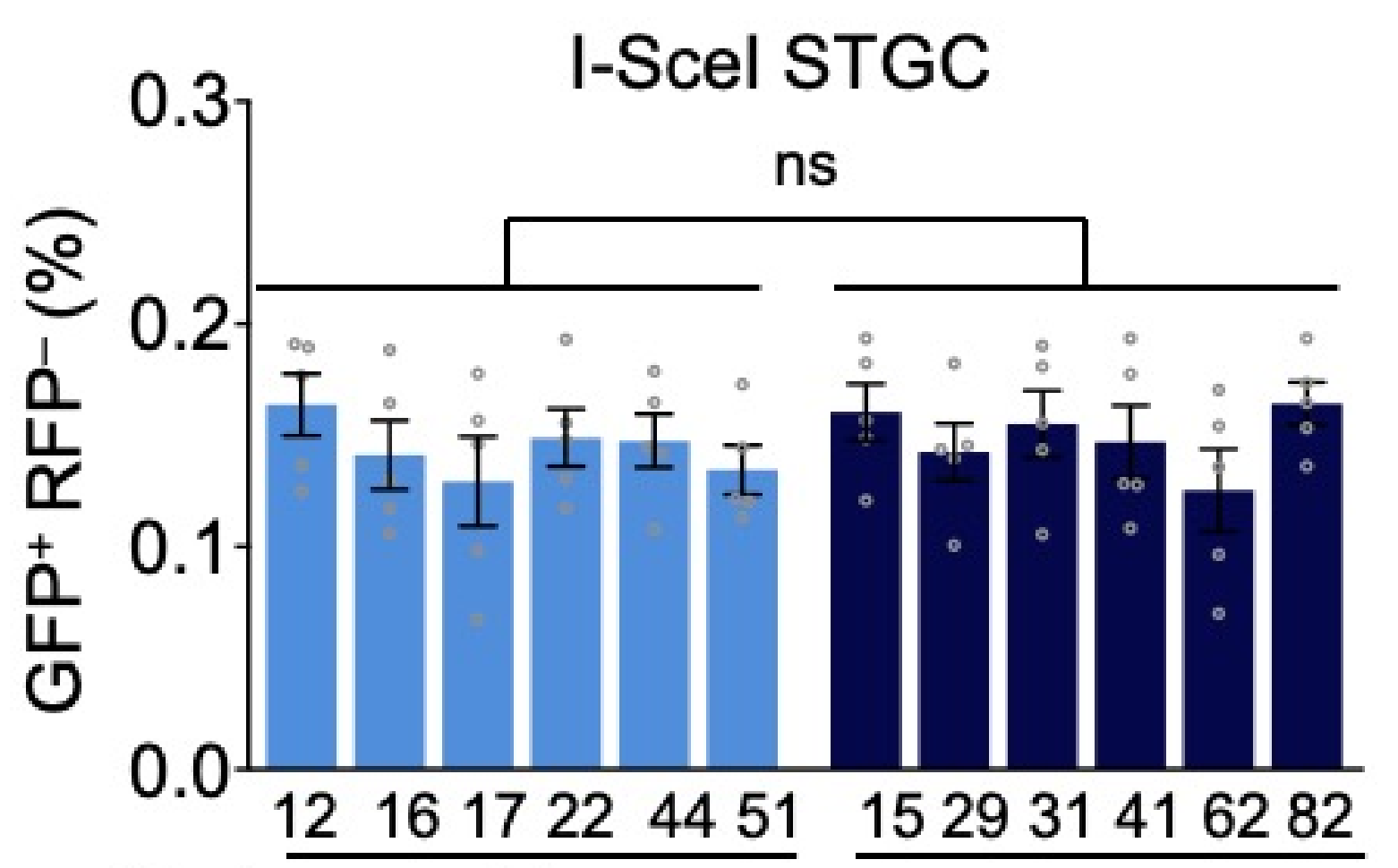

S/X4:

$\triangle \mathrm{SBD} /-$ 
a

SLX4 $\triangle M L R$
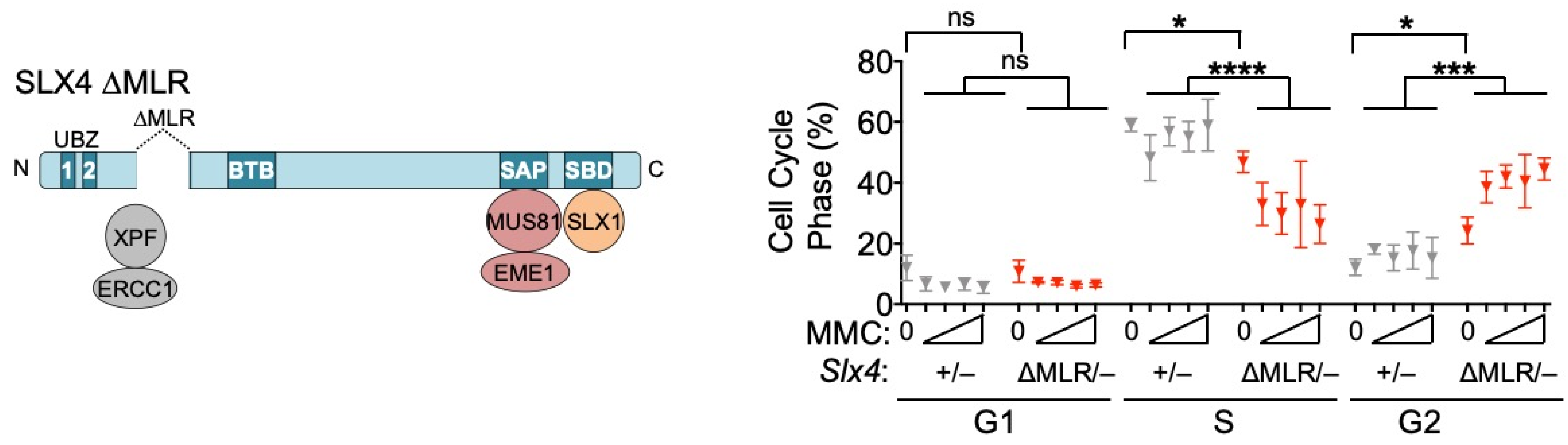

C
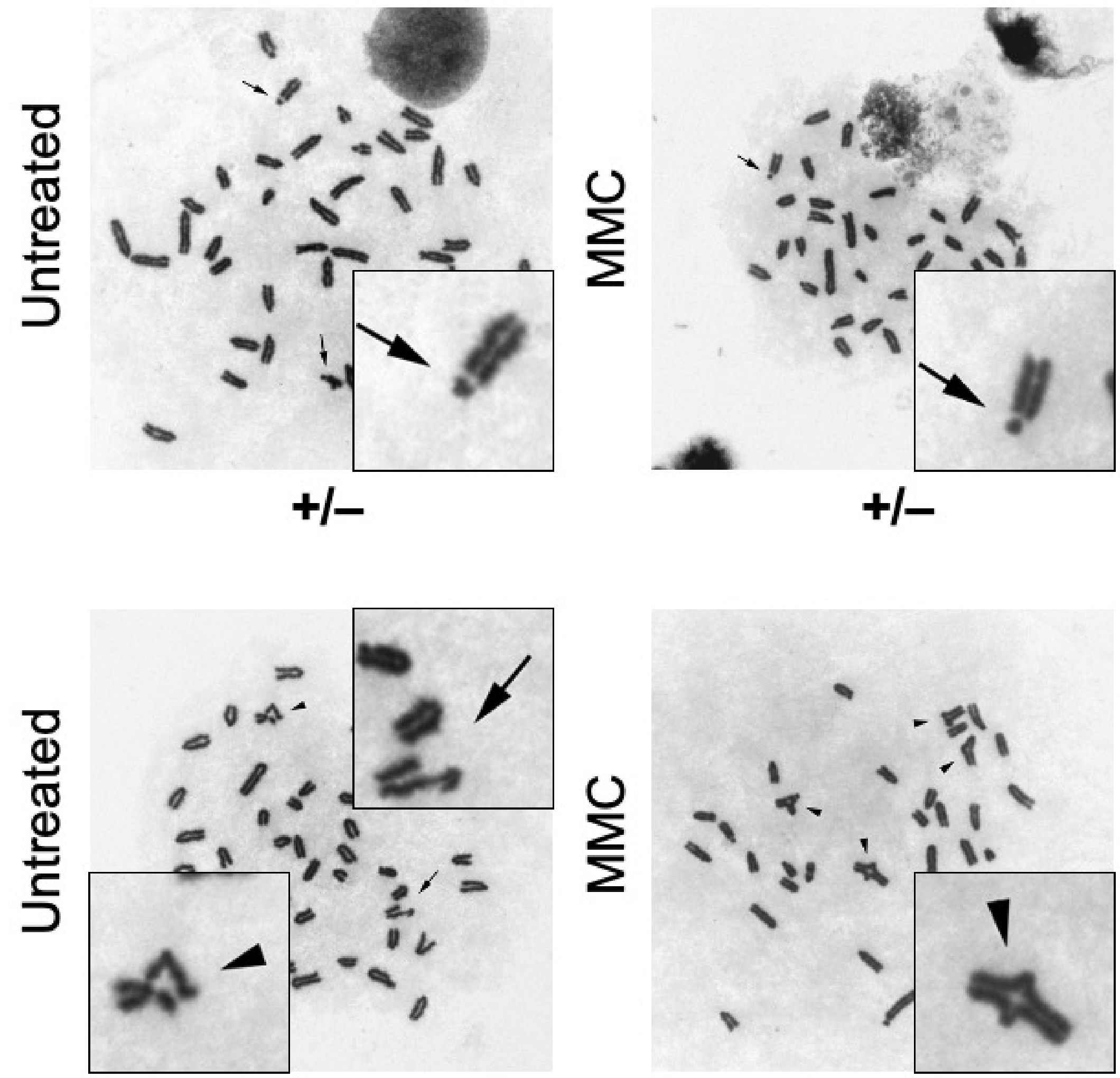

$\triangle$ UBZI-

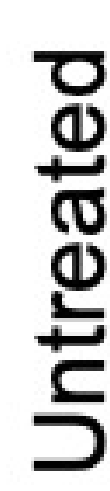

$\Delta \mathrm{MLR} /-$

$\Delta$ UBZI-

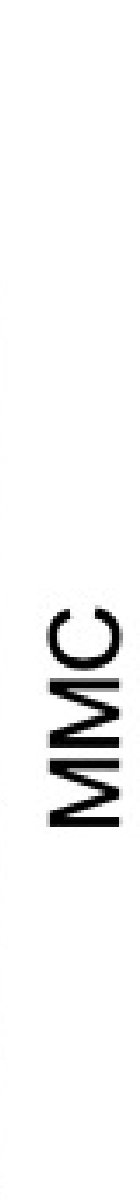

काल

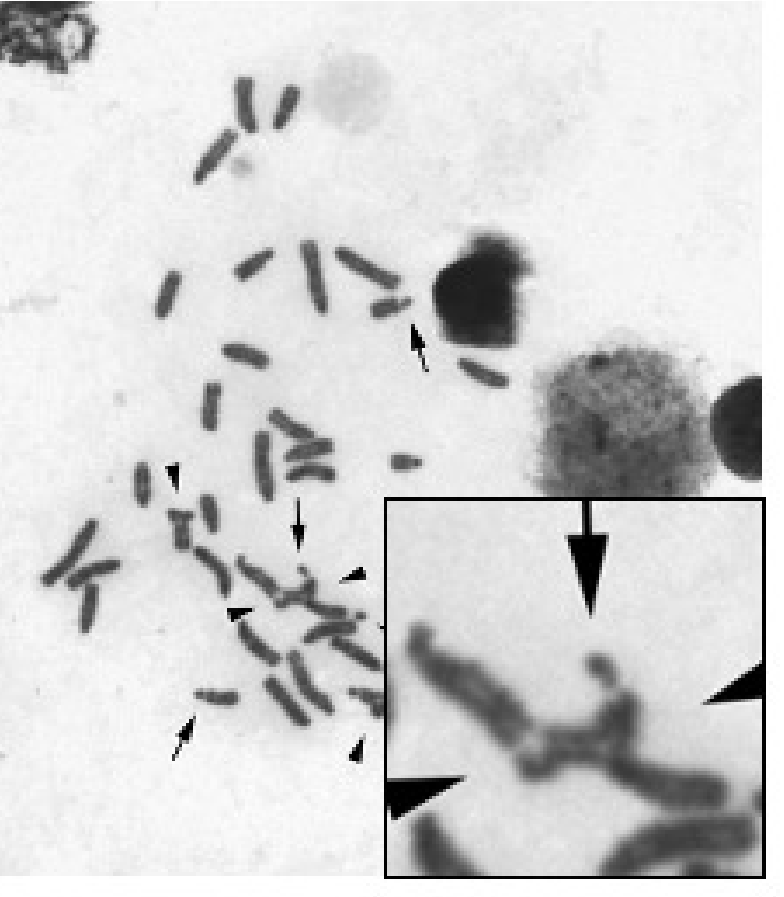

$\Delta \mathrm{MLR} /-$ d
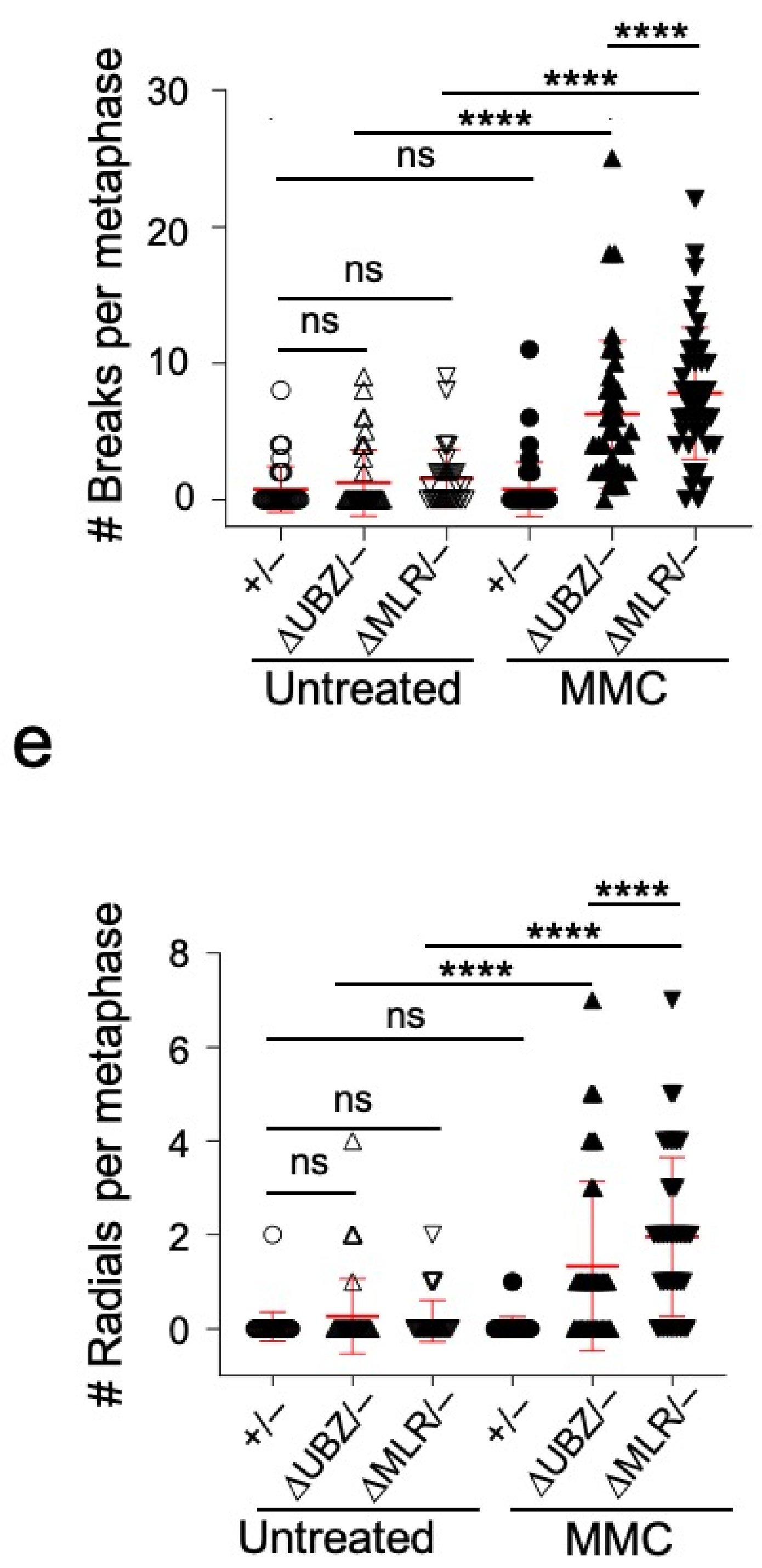

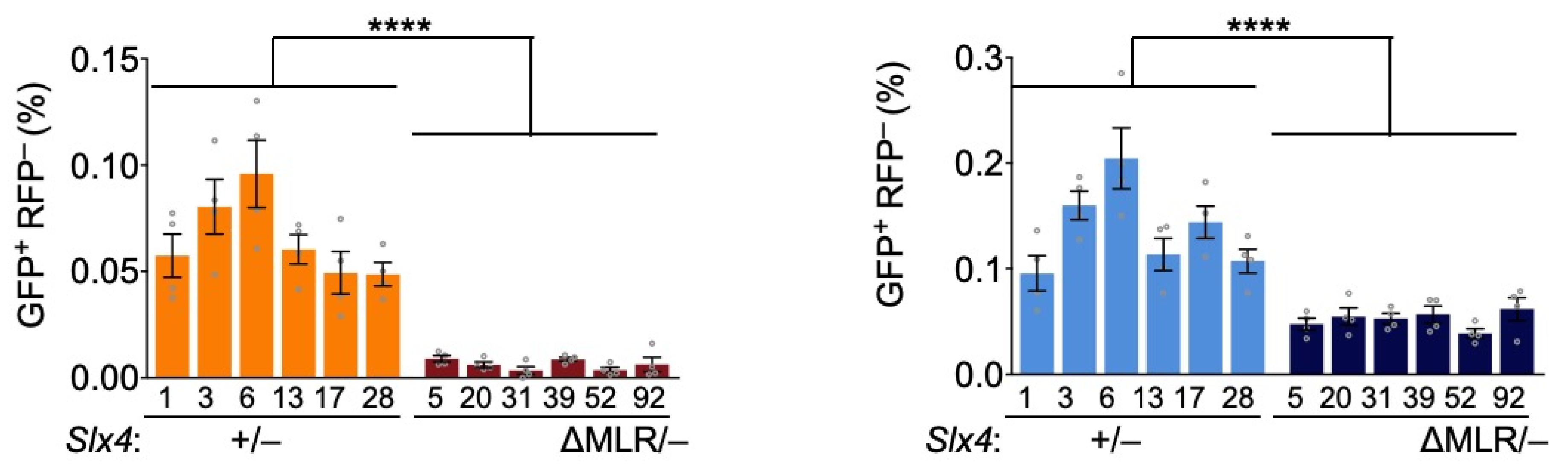

d

SLX4 RTqPCR

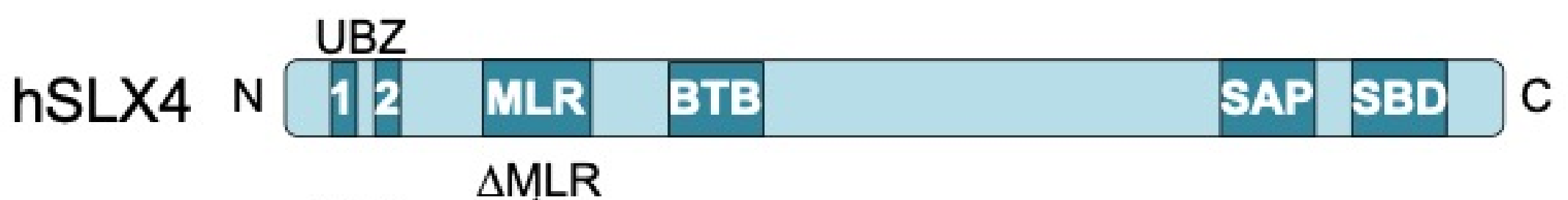

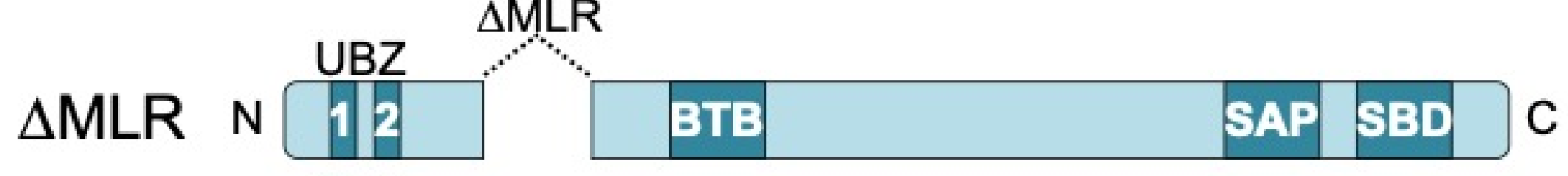

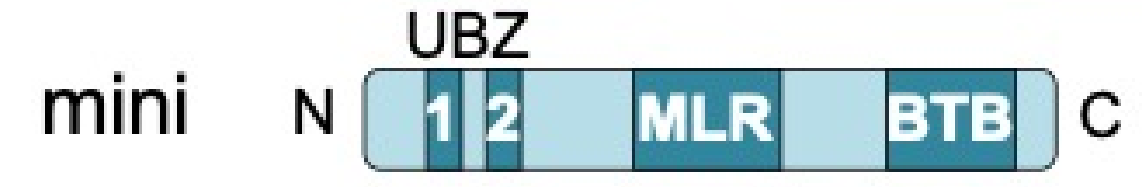

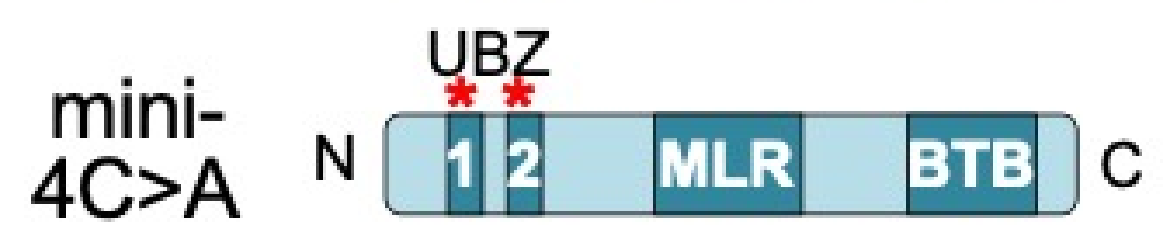

$\underset{\Delta M L R}{\operatorname{mini}} \stackrel{\text { UBZ }}{\triangle 1} \stackrel{ }{\triangle M L R}$
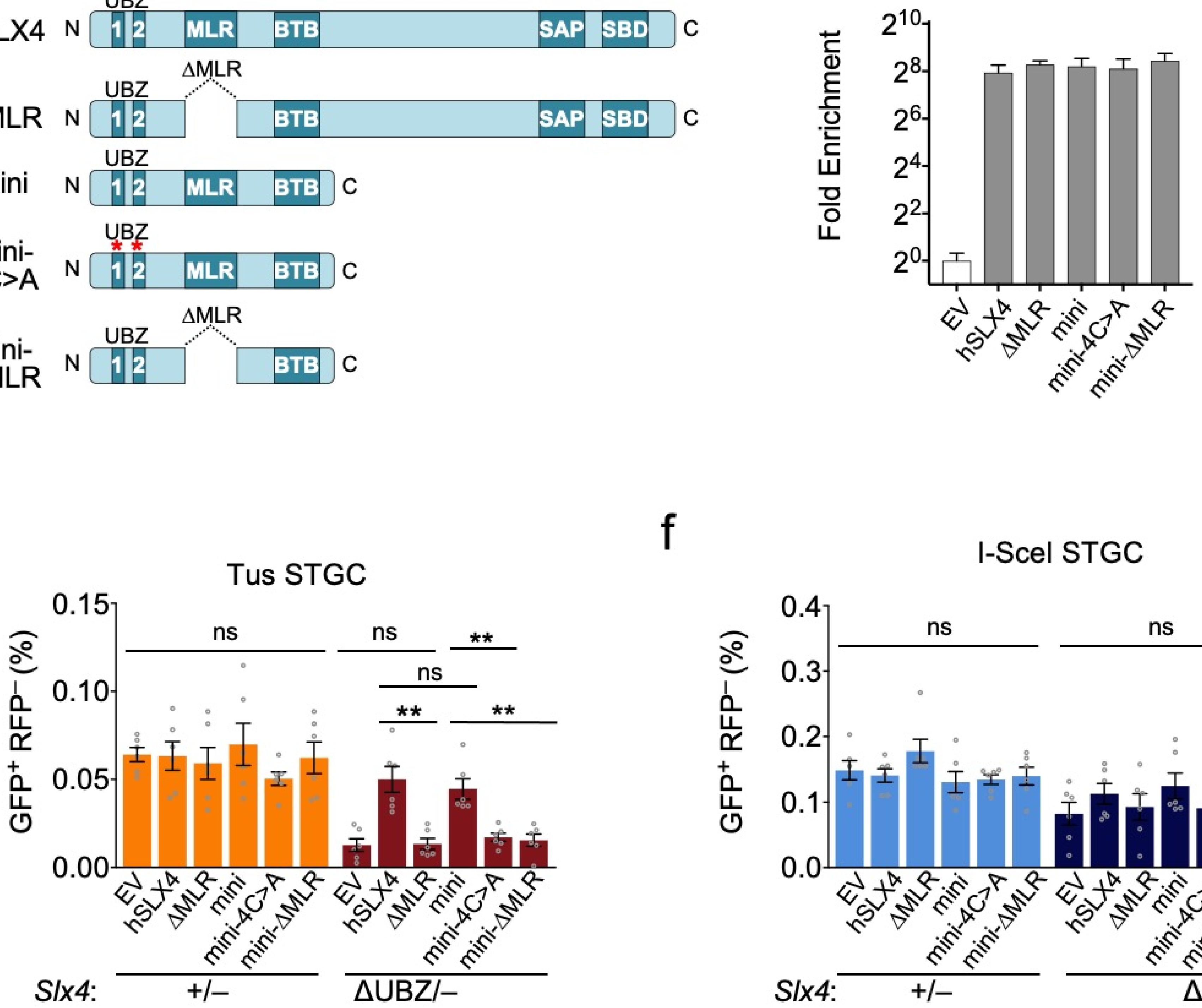

f

I-Scel STGC

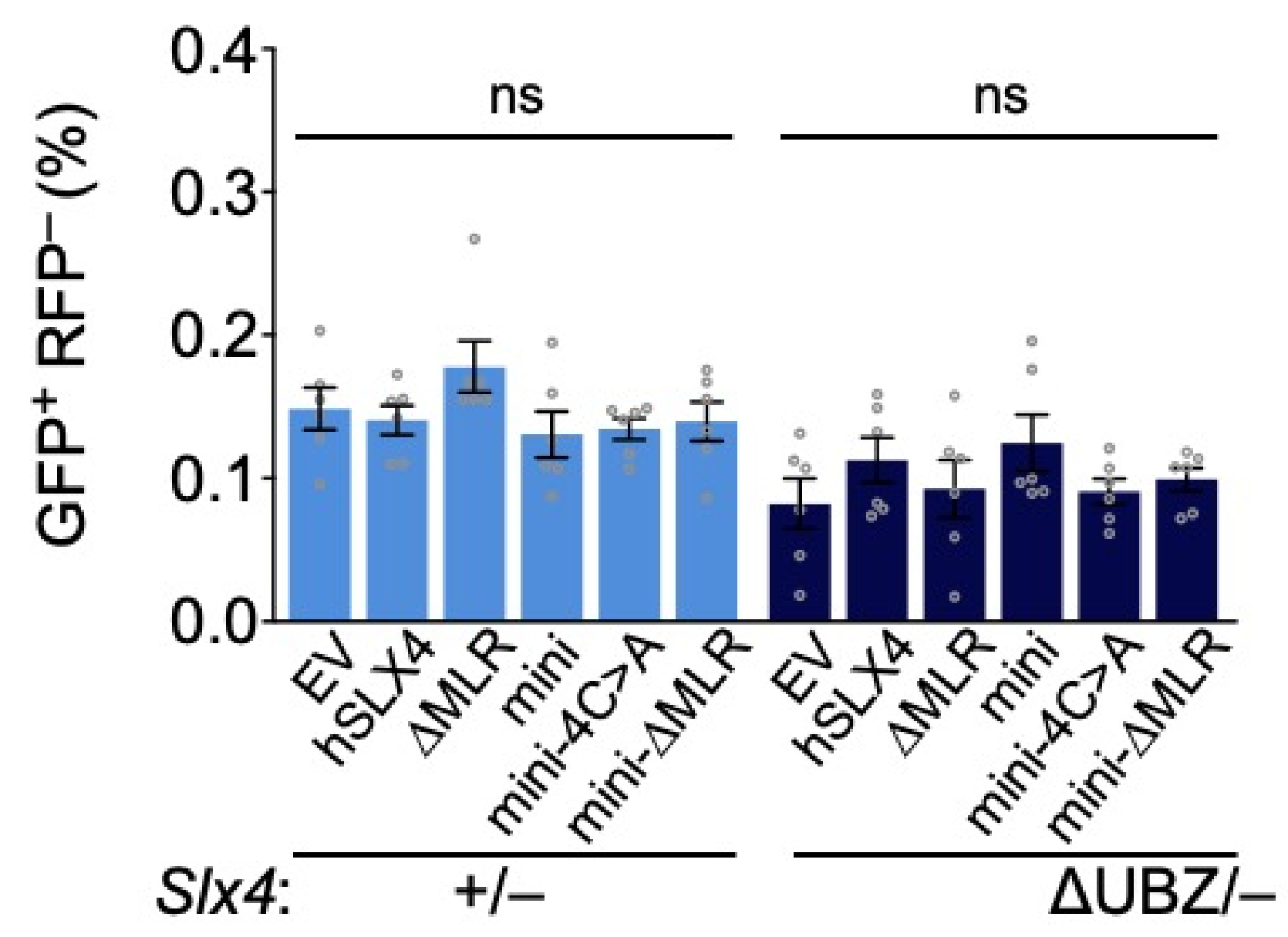


M.m. XPF

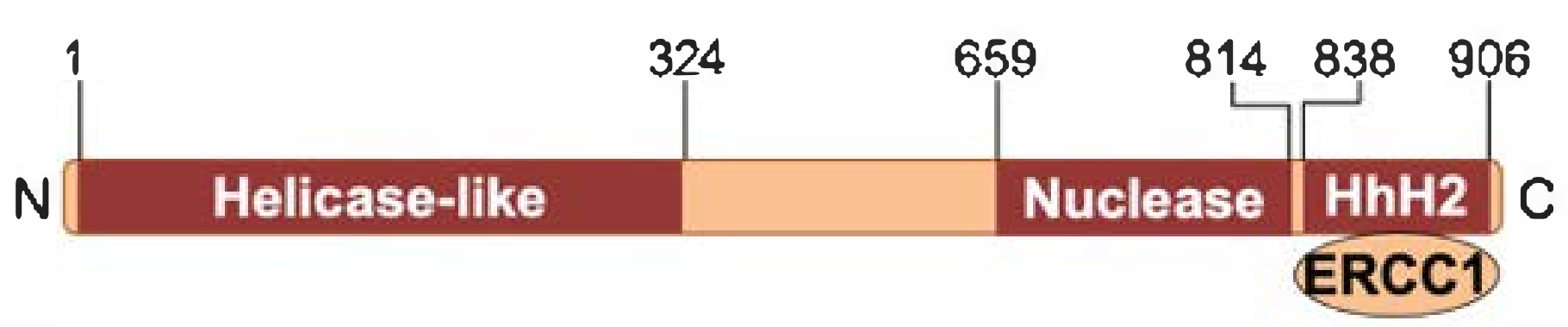

$\Delta$ Nuclease

\section{Helicase-like}

HhH2 C

ERCC1

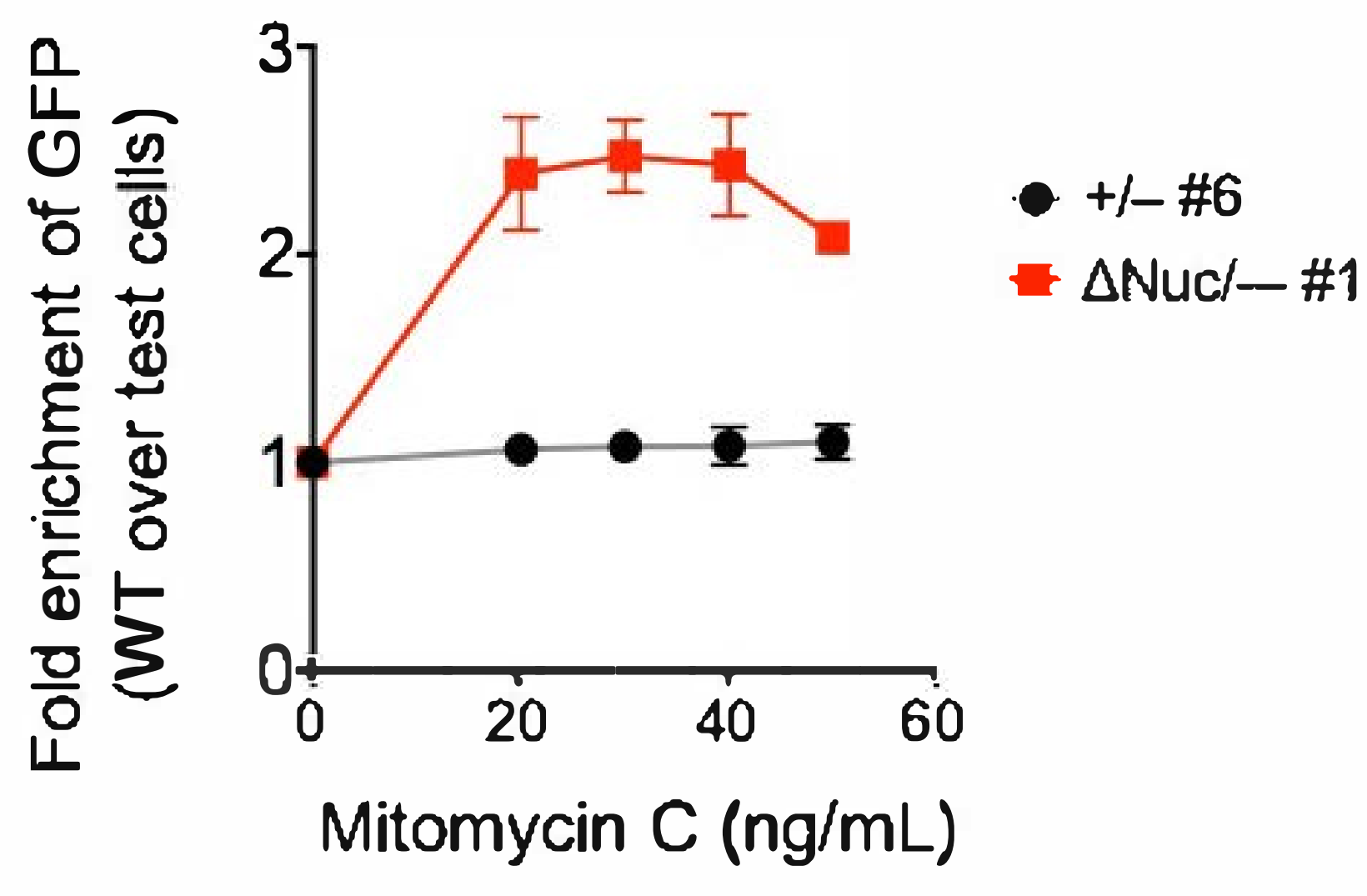

d

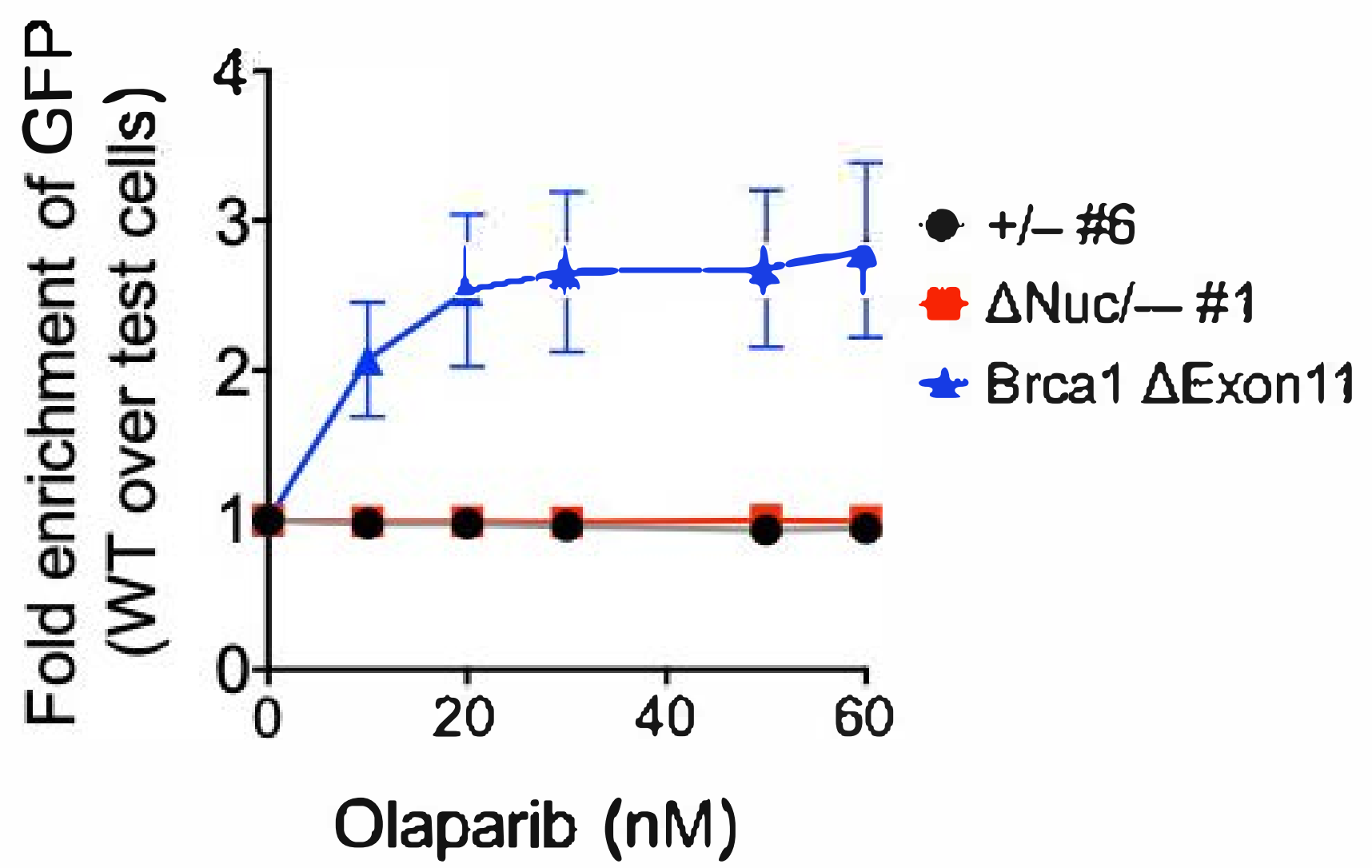

f

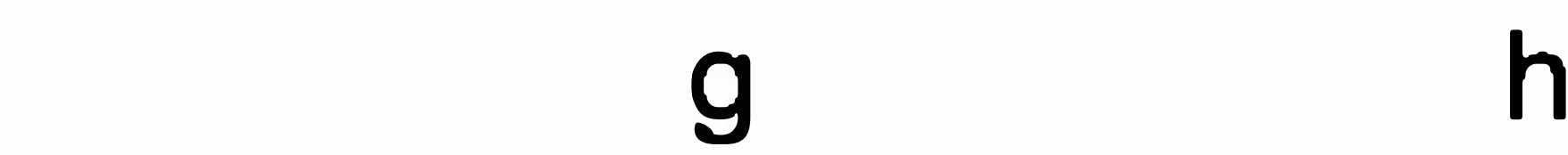

(1)

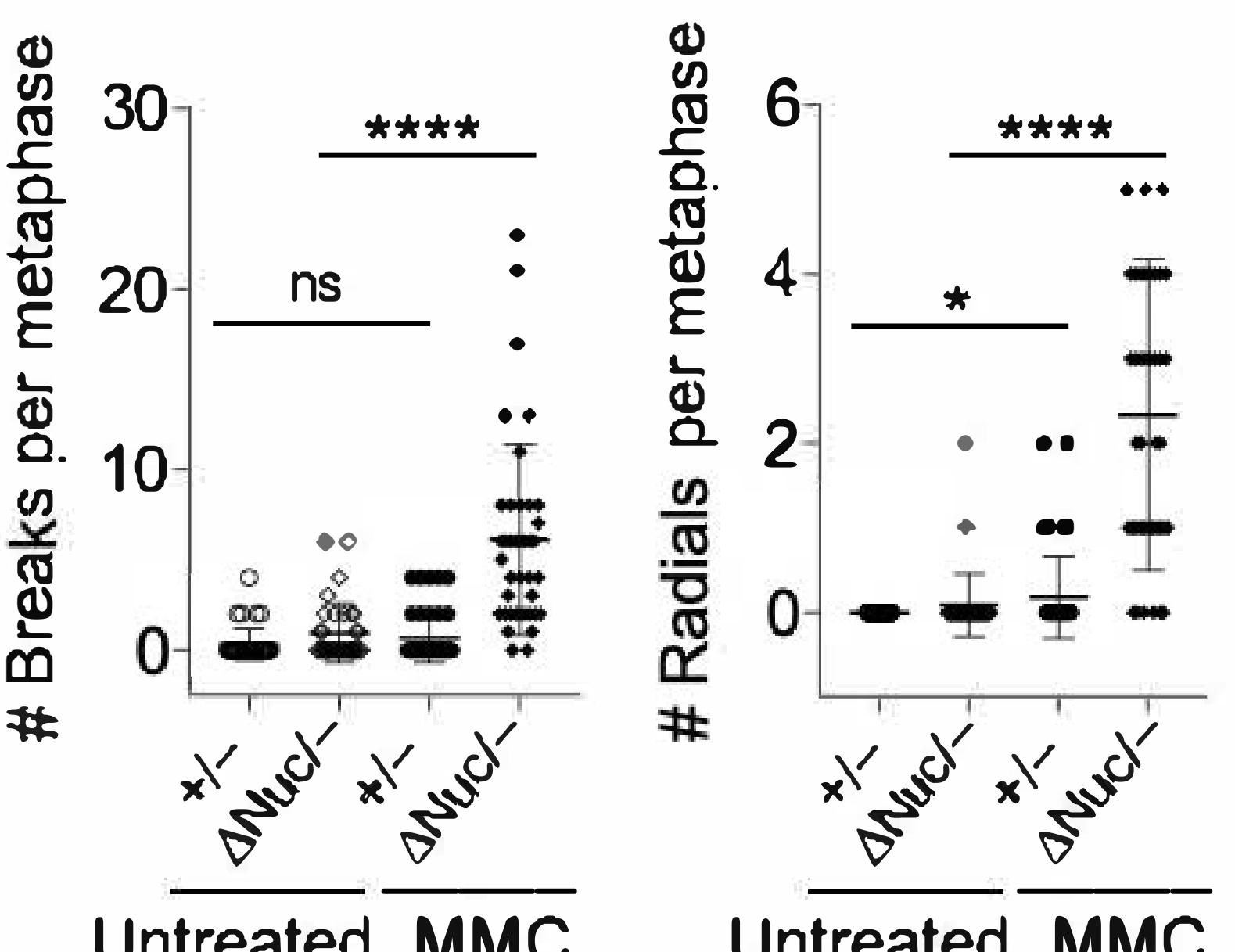

h

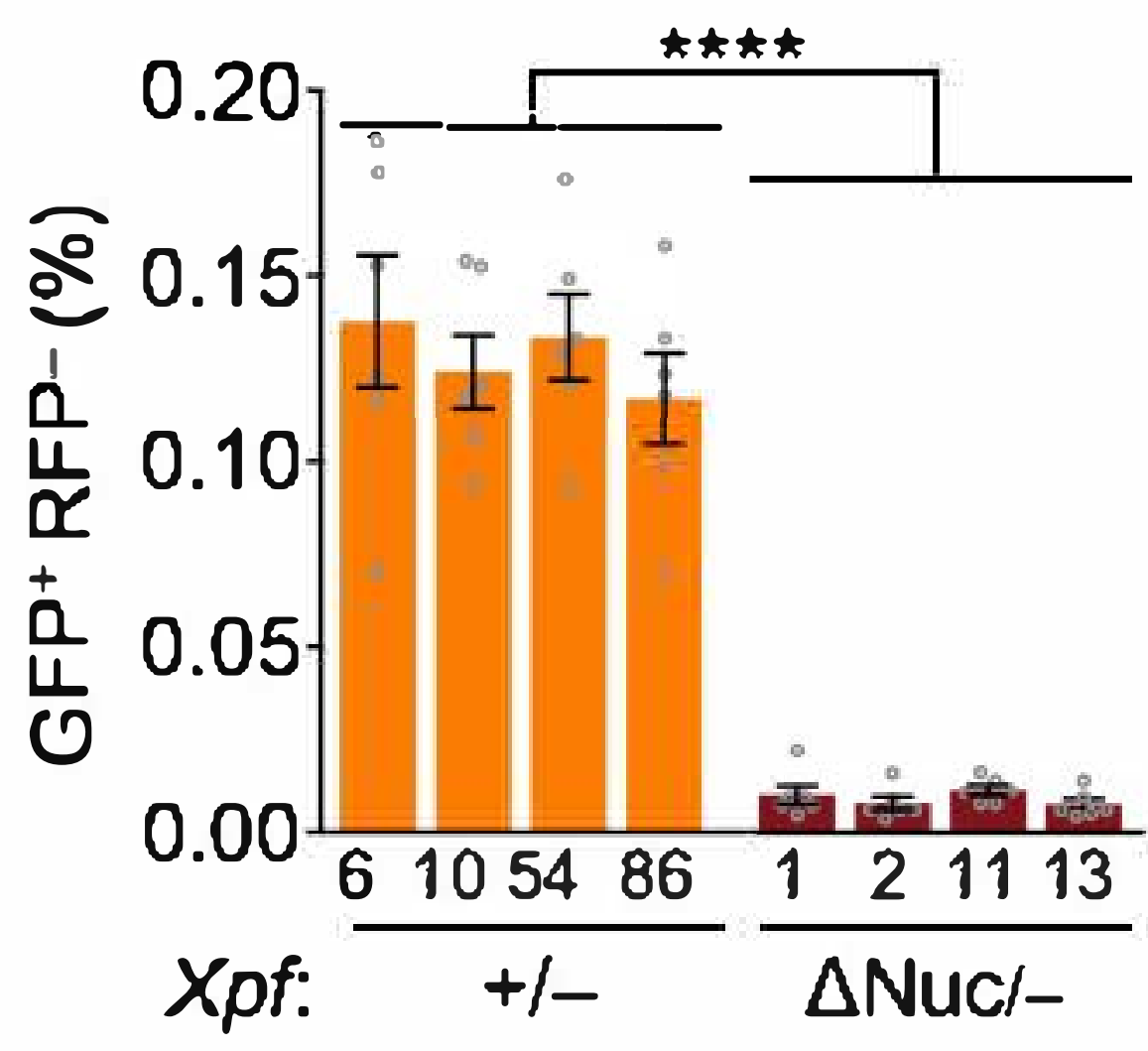

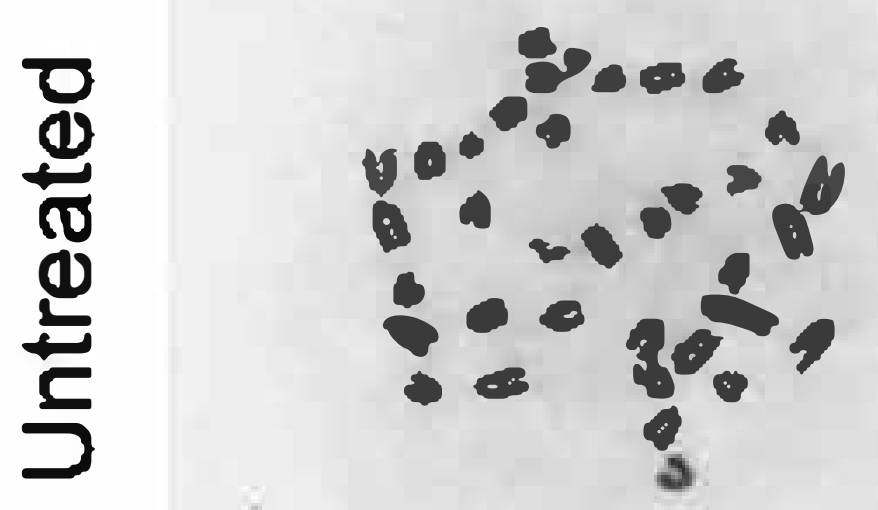

Tus STGC

sgRnA2 Fig 6 Nuc-F' $\rightarrow \downarrow$ Exon11 FNuc-R $\Delta N u c$

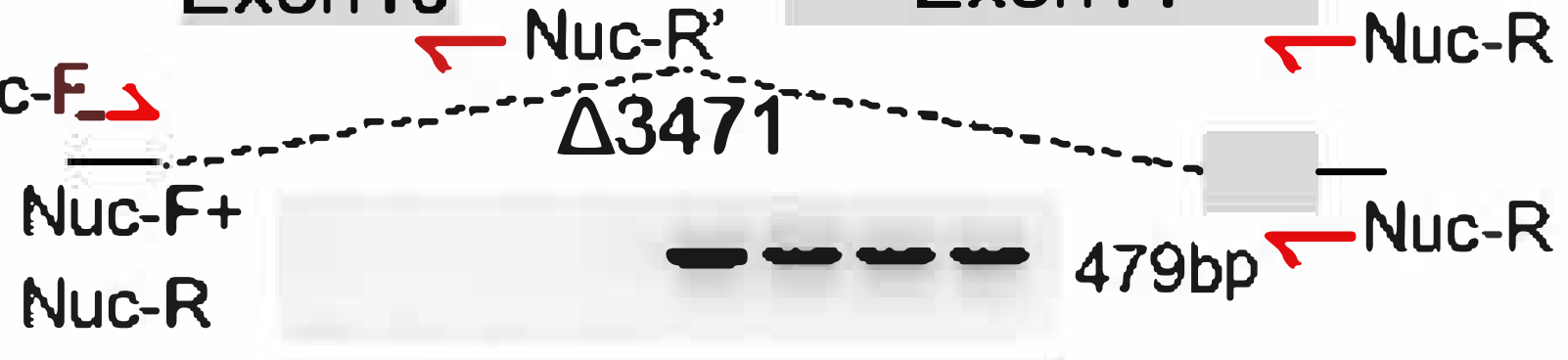

Nuc-F+

Nuc-F'+

$444 b p$

Nuc-R

$$
\text { Xpf: } \frac{6105486}{+/-} \frac{121113}{\Delta \text { Nuc/- }}
$$

'ATTAATCTAGAACGTTGCGGCTGCTCTGGT 3

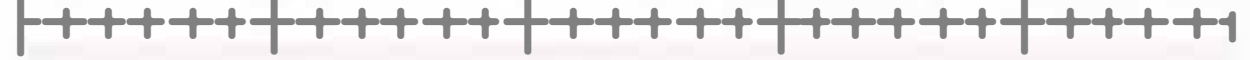

- _ - . $R$ L L W C

ATtAATCTAGAACGTTGCGGCTGCTCTGGT

$537 b p$

e
$+1-$

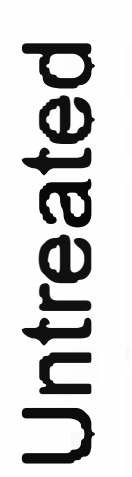

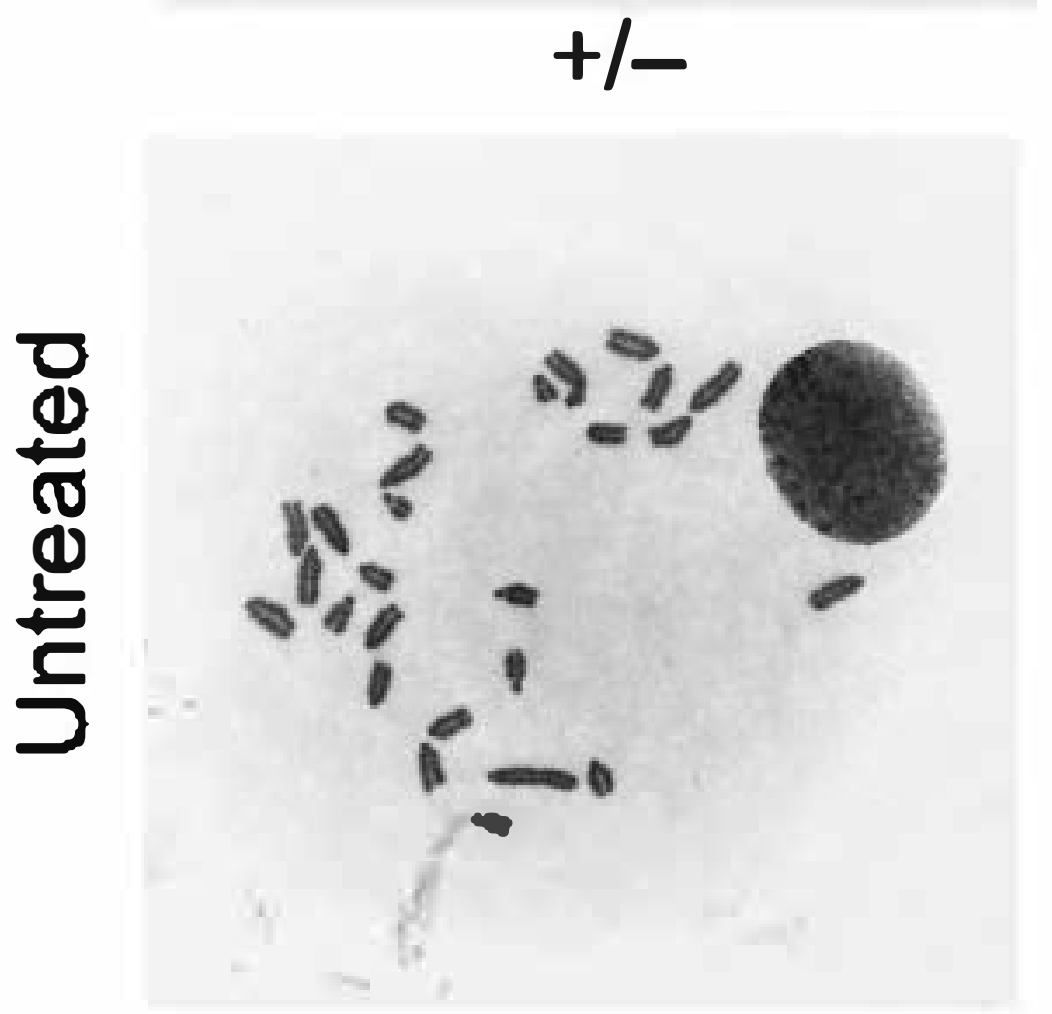

$\Delta \mathrm{Nucl}-$
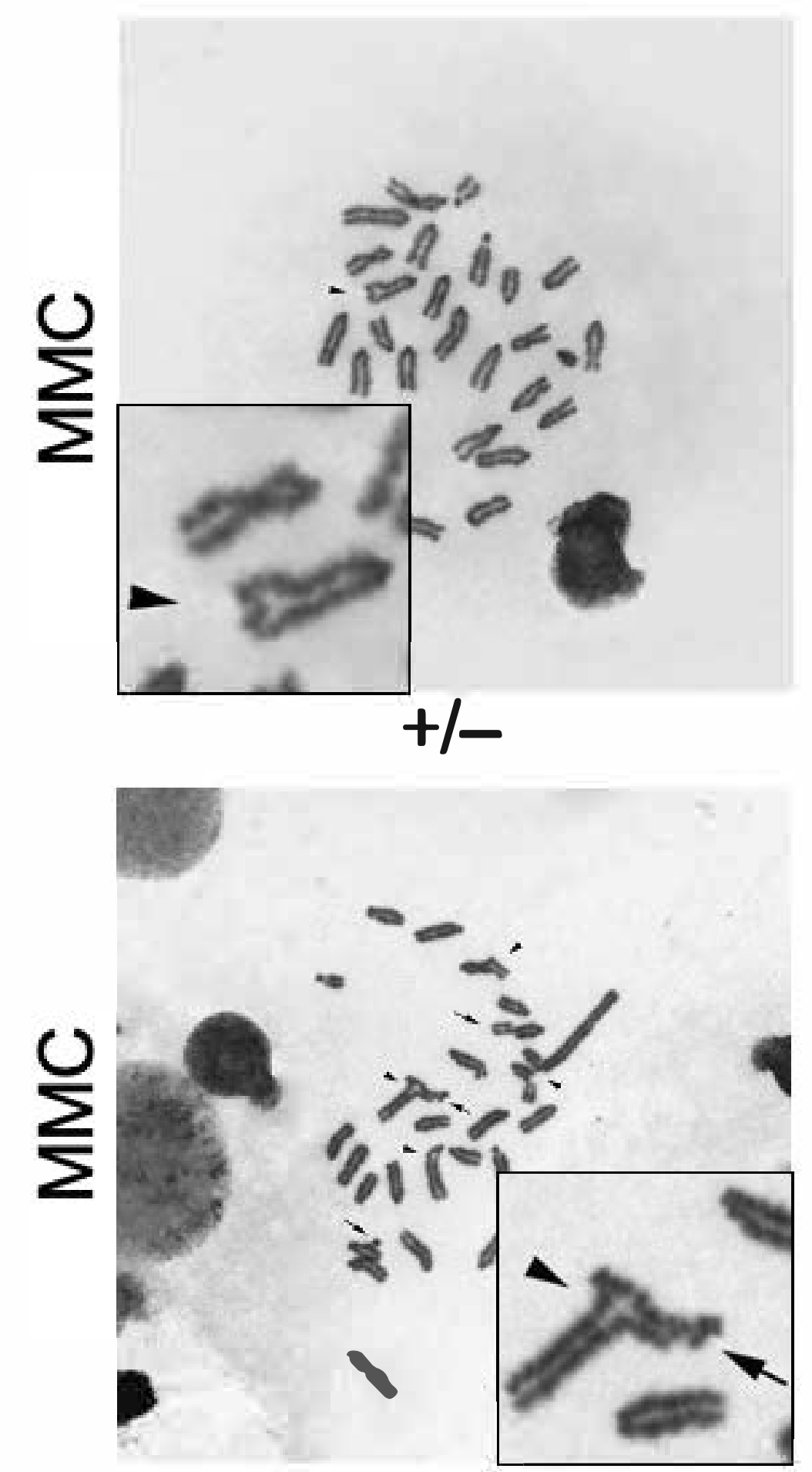

$\Delta \mathrm{NuCl}-$
I-Scel STGC

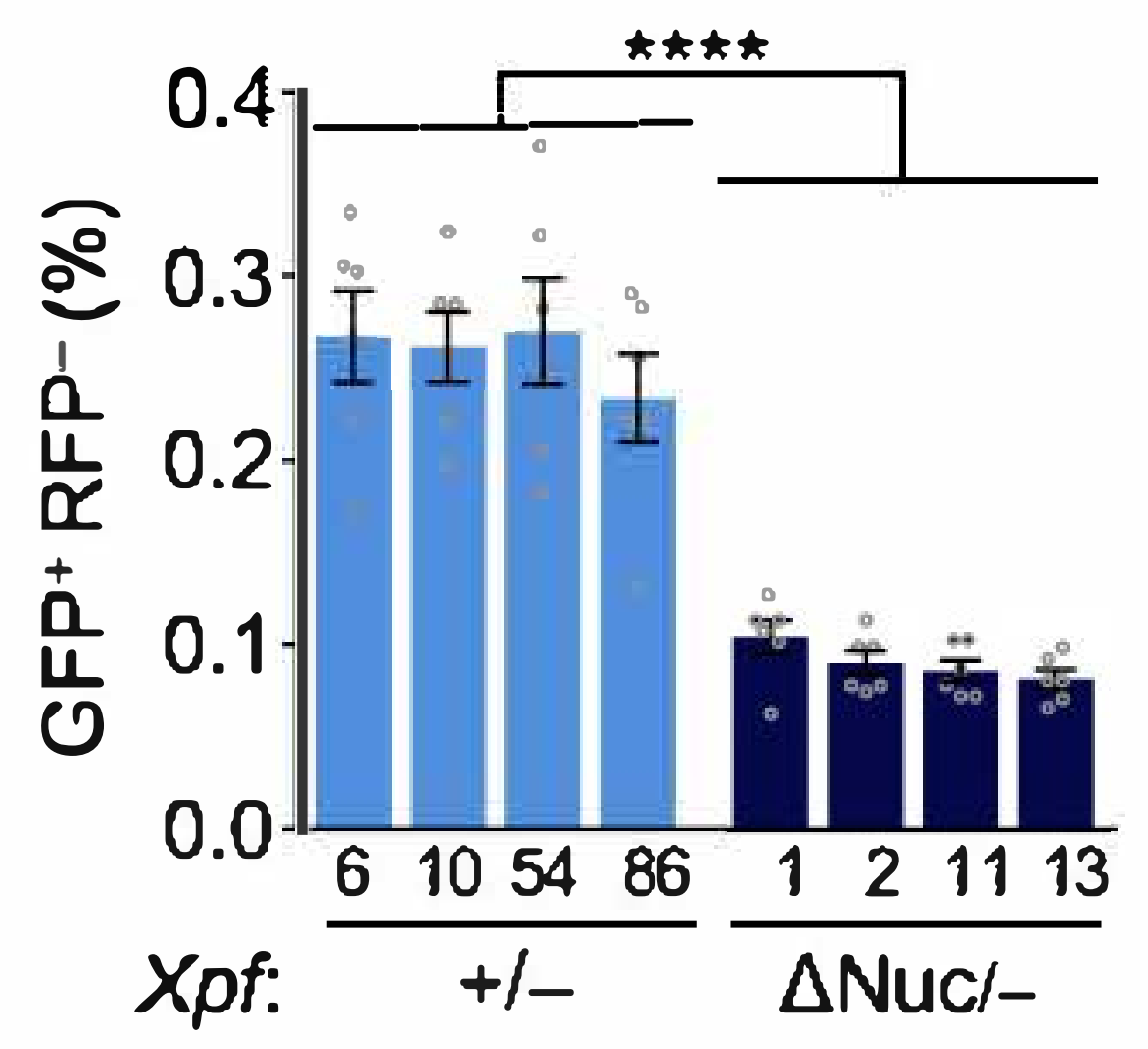


a

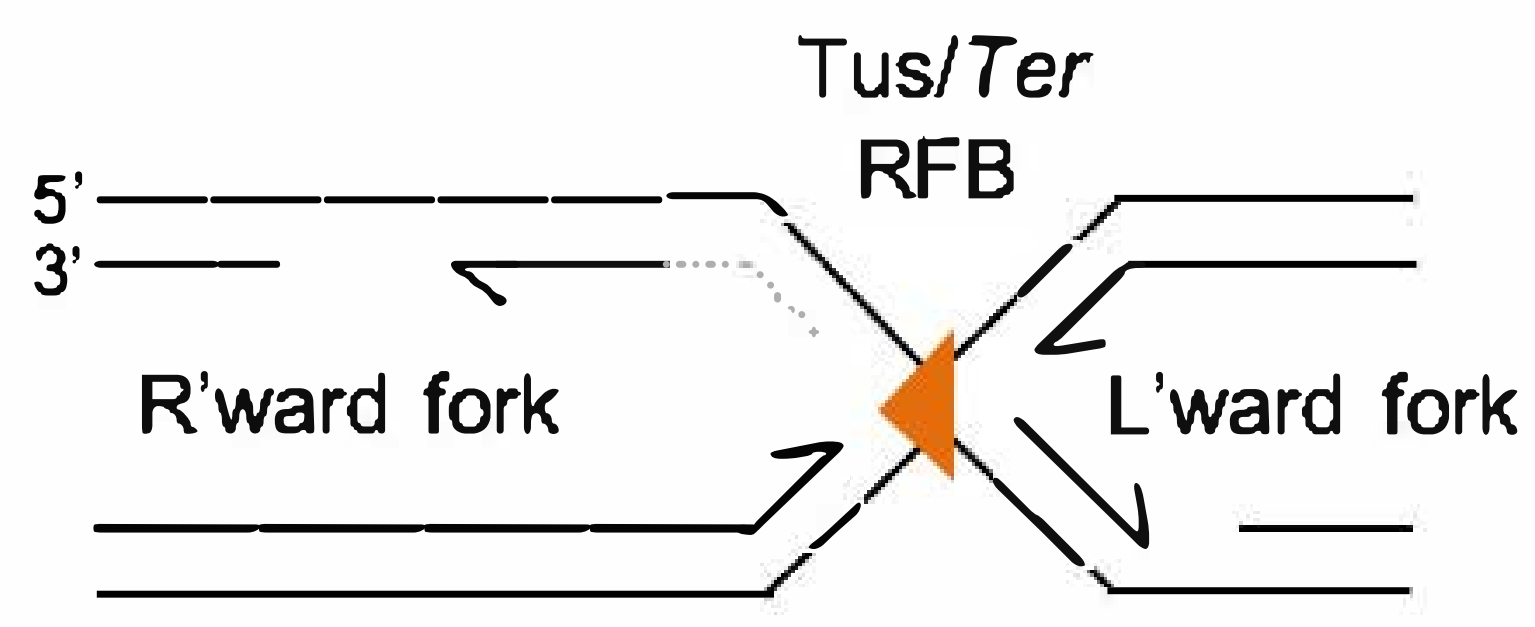

FA pathway activation Fork Reversal
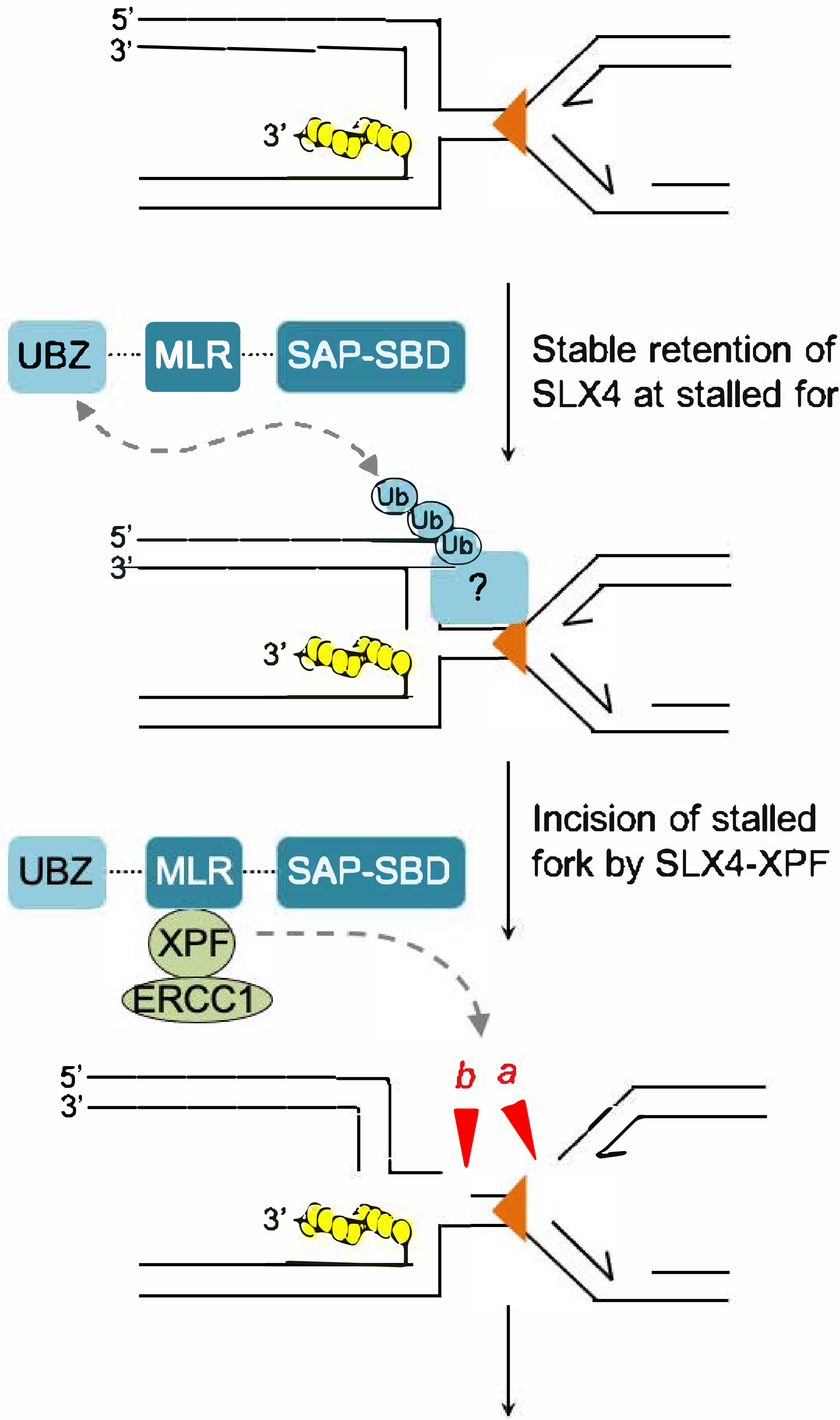

Efficient STGC b

Fig 7

\section{$\triangle \mathrm{UBZ}$ \\ MLR .... SAP-SBD}

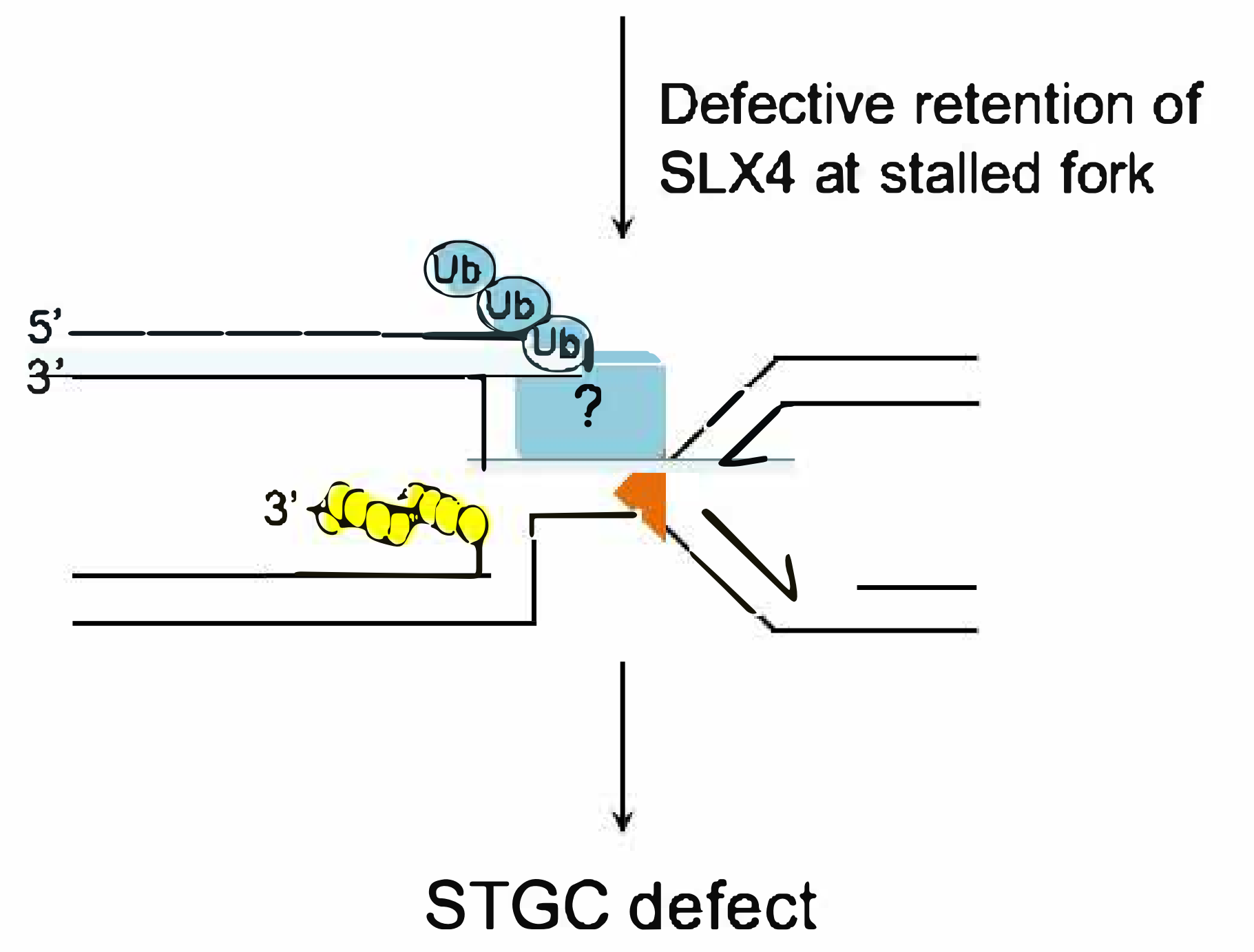

C
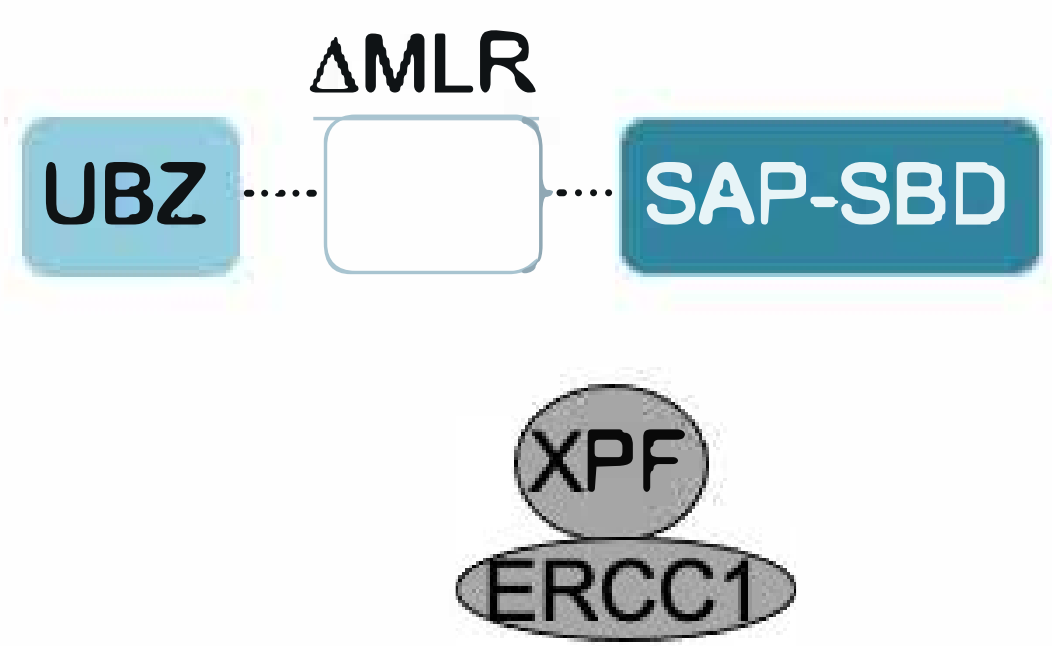

Absence of XPFmediated incisions

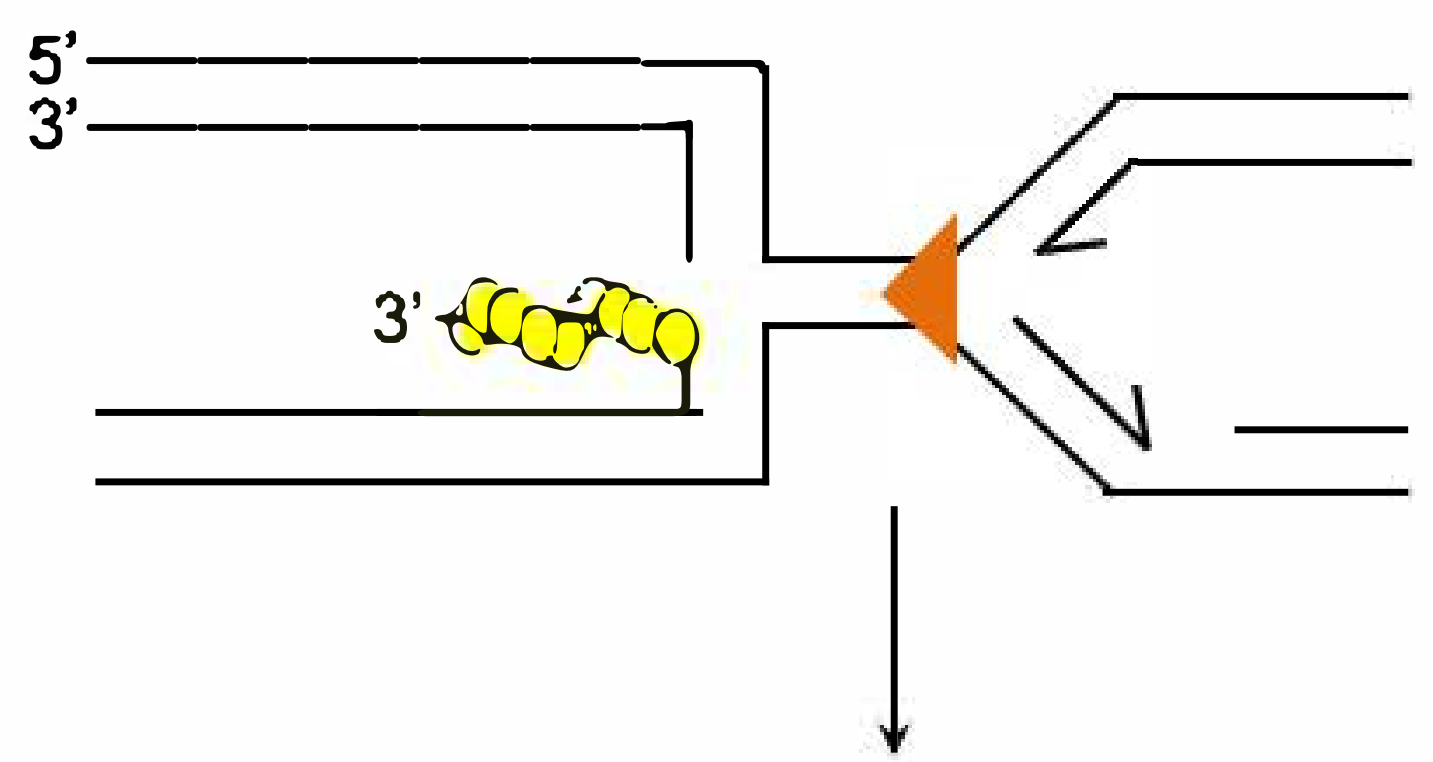

Severe STGC defect 


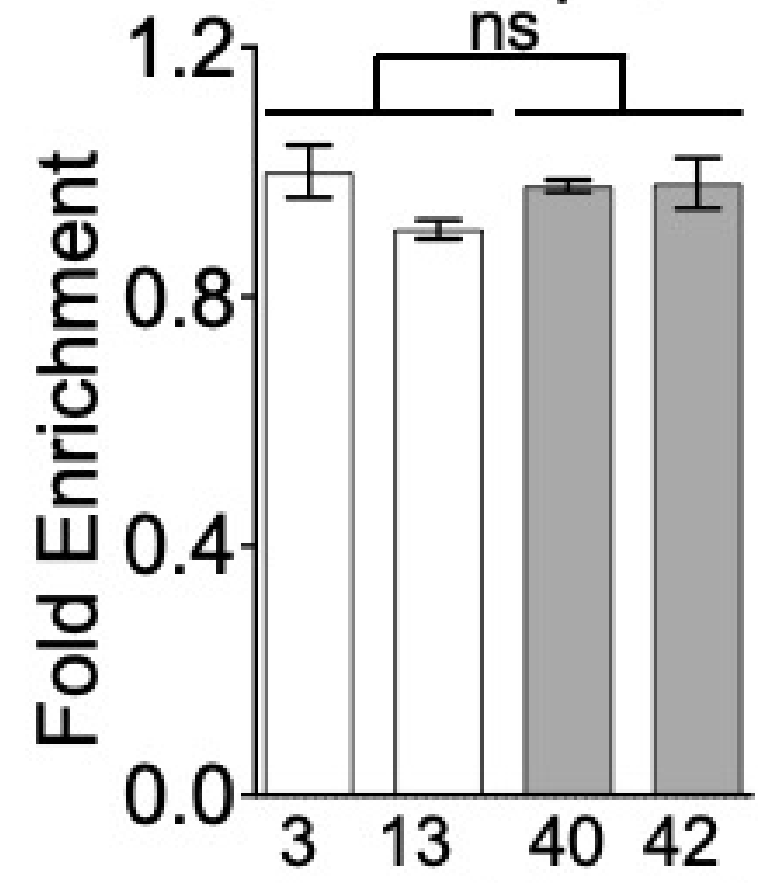

SIX4: $\frac{3 \quad 13}{+/+} \frac{4042}{\Delta 125 / \Delta}$

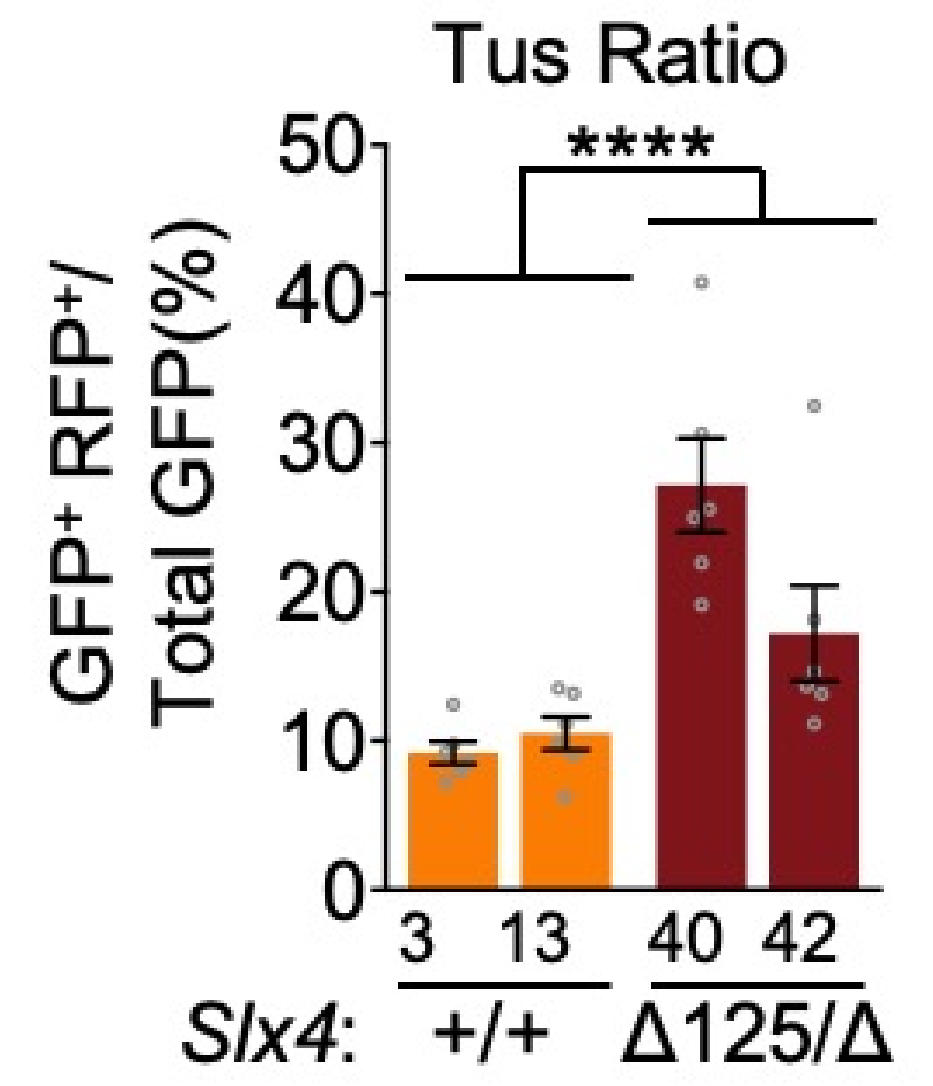

C Suppl Fig 1 d

\section{(5)}

$F_{7} \downarrow$ 564bp $\sqrt{5^{\prime} \mathrm{R}}$

\section{Exon 2}

SIX4

$+1+$

2
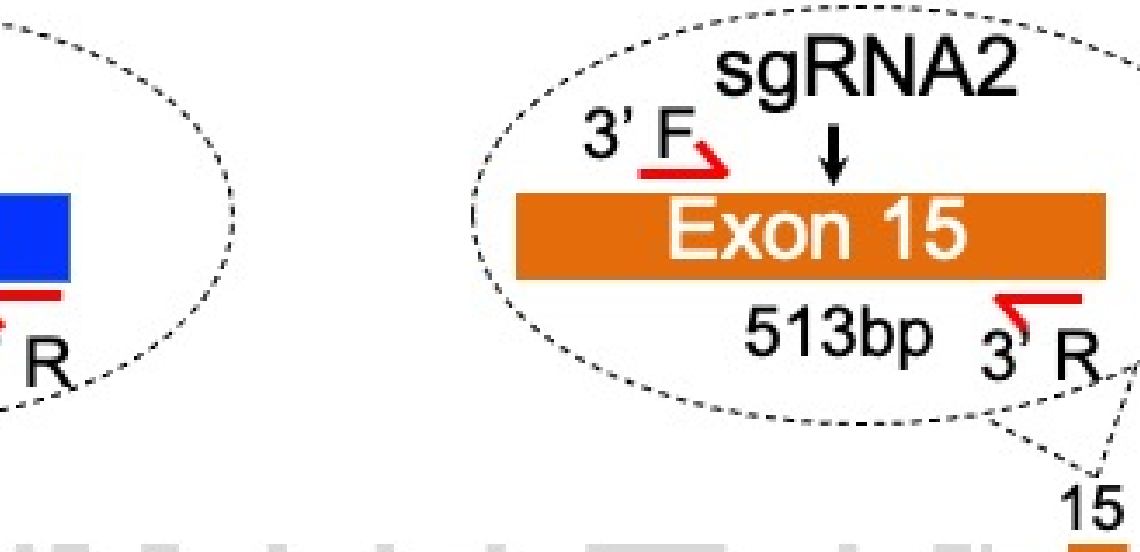
$\Delta 19.3 \mathrm{~kb}$

f
\# of clones

Total

202/262

$60 / 262$

$0 / 262$

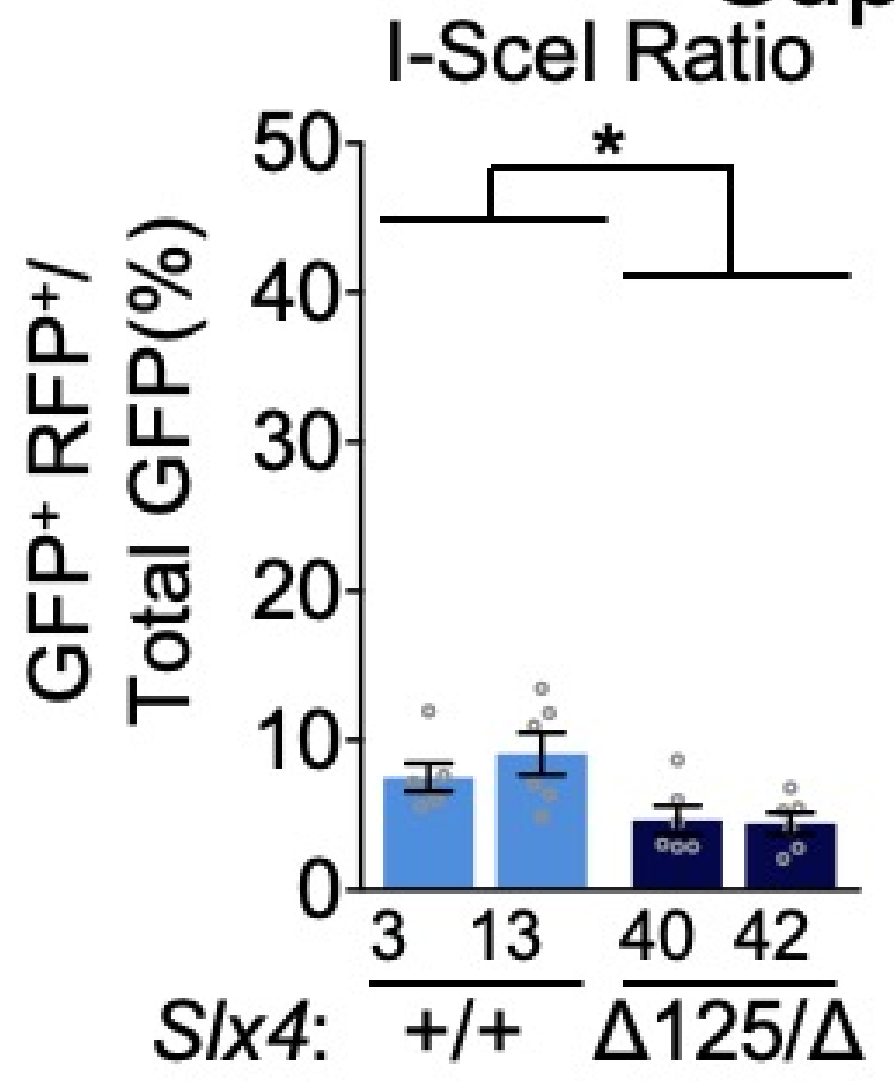

\section{SIX4 +1-}

Exon 2

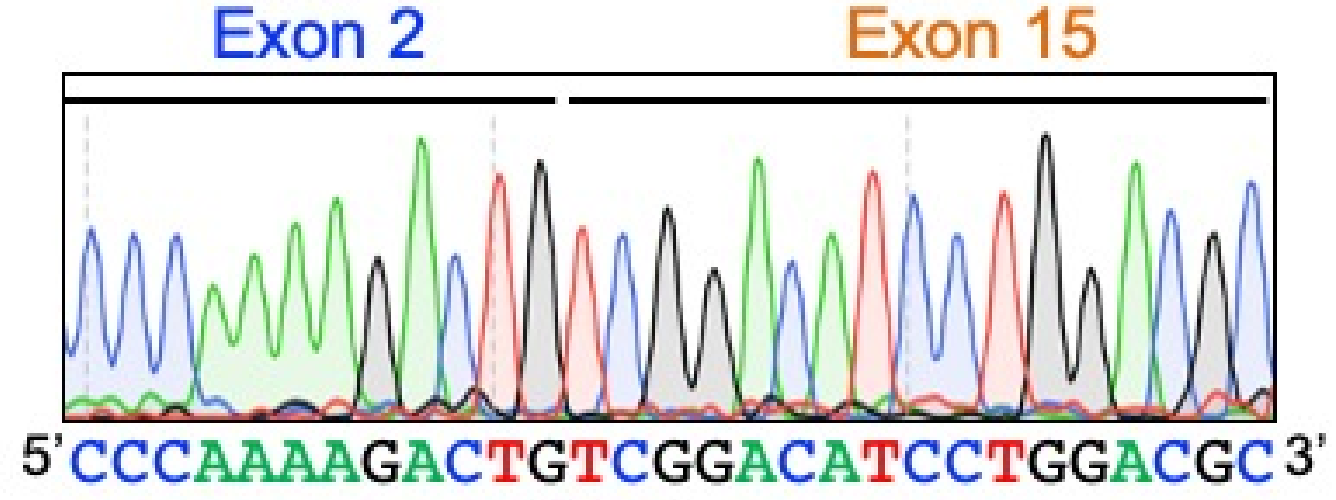
SIX4 -

g

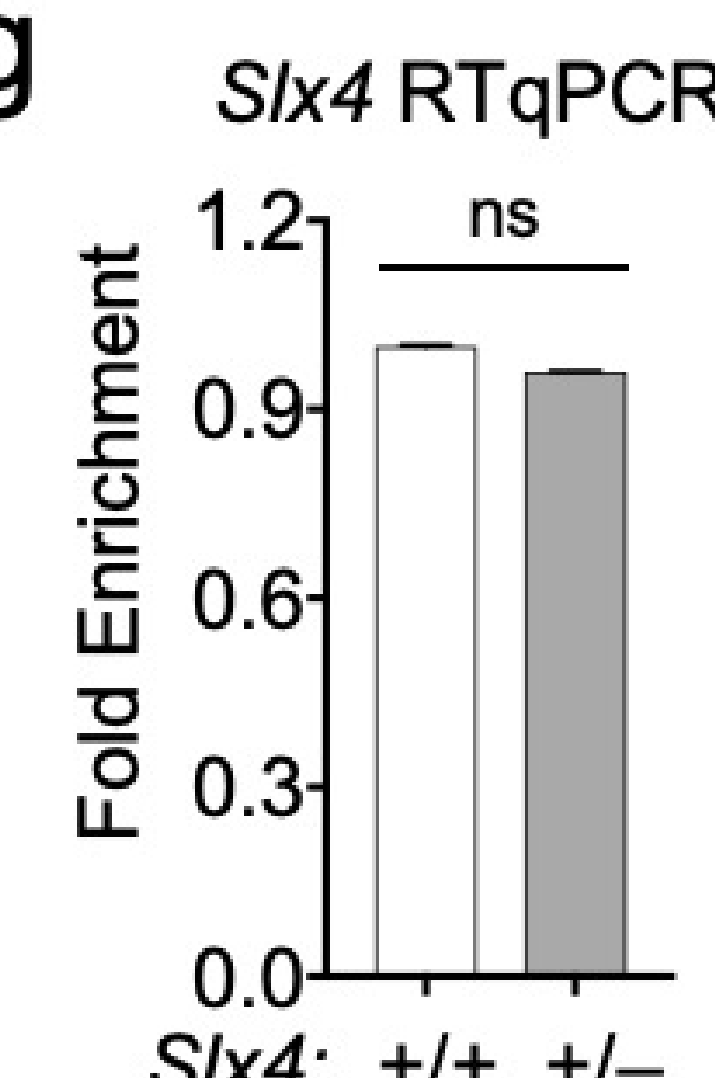

SIX4: $+1++1-$

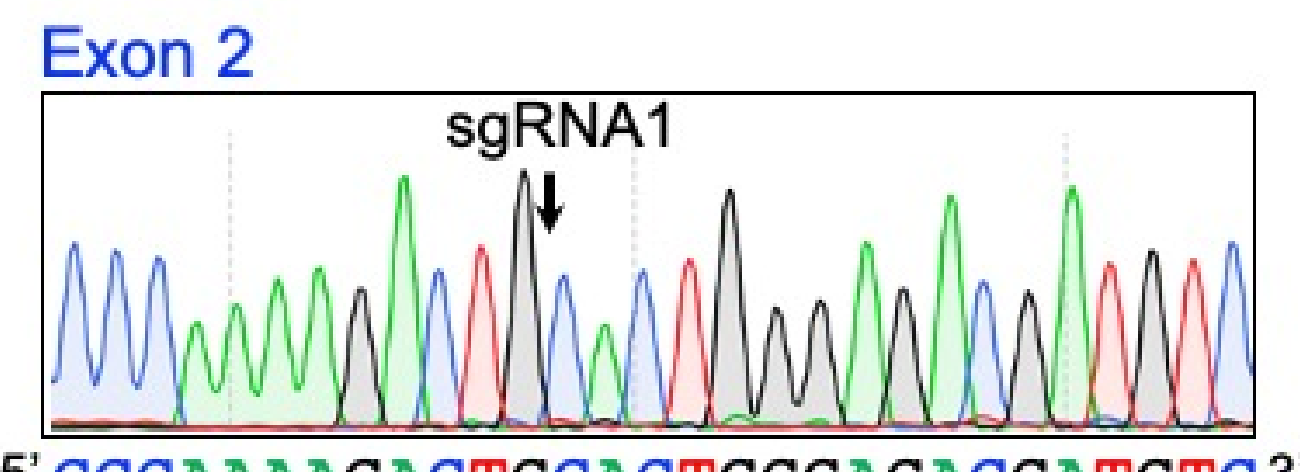
SIx4 +

\section{Exon 15

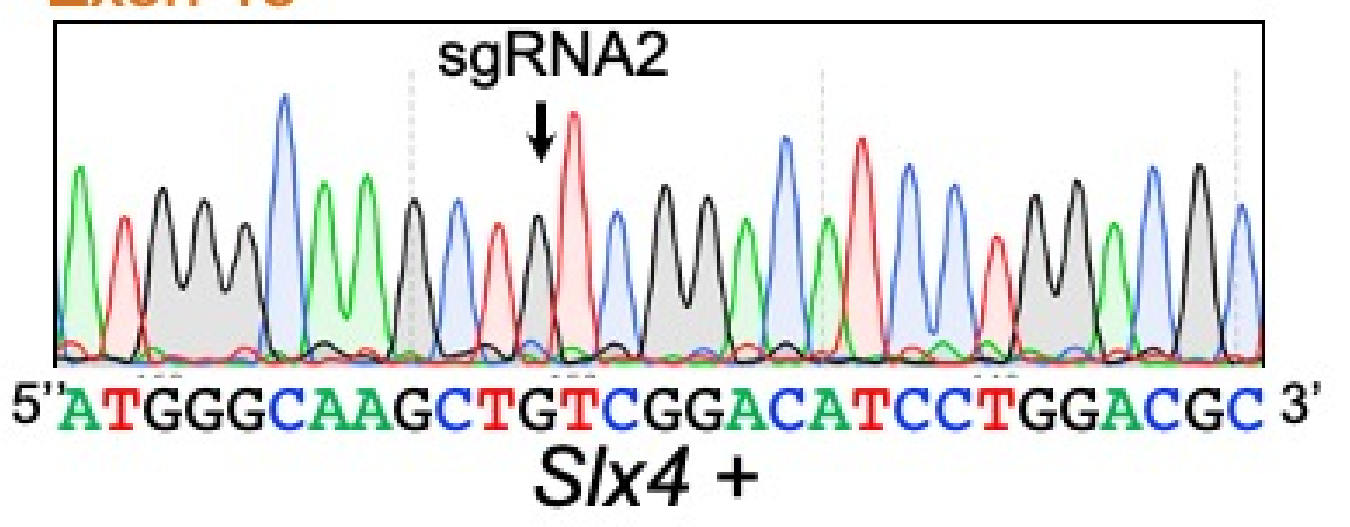

h

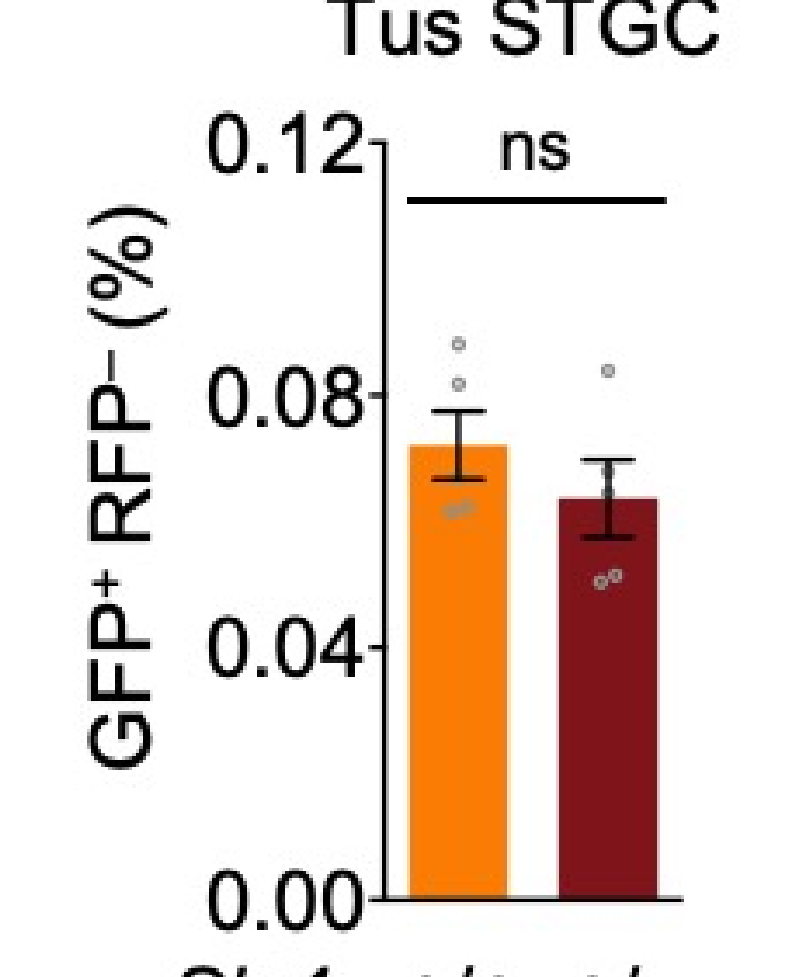

Slx4: +1+ +1-

I-Scel STGC

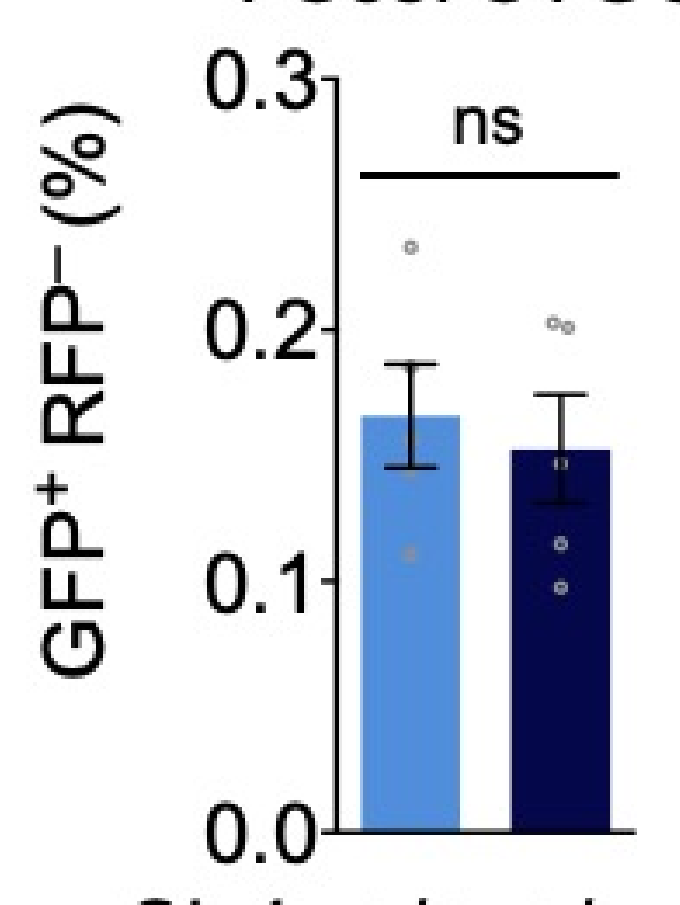

Slx4: +1+ +1-

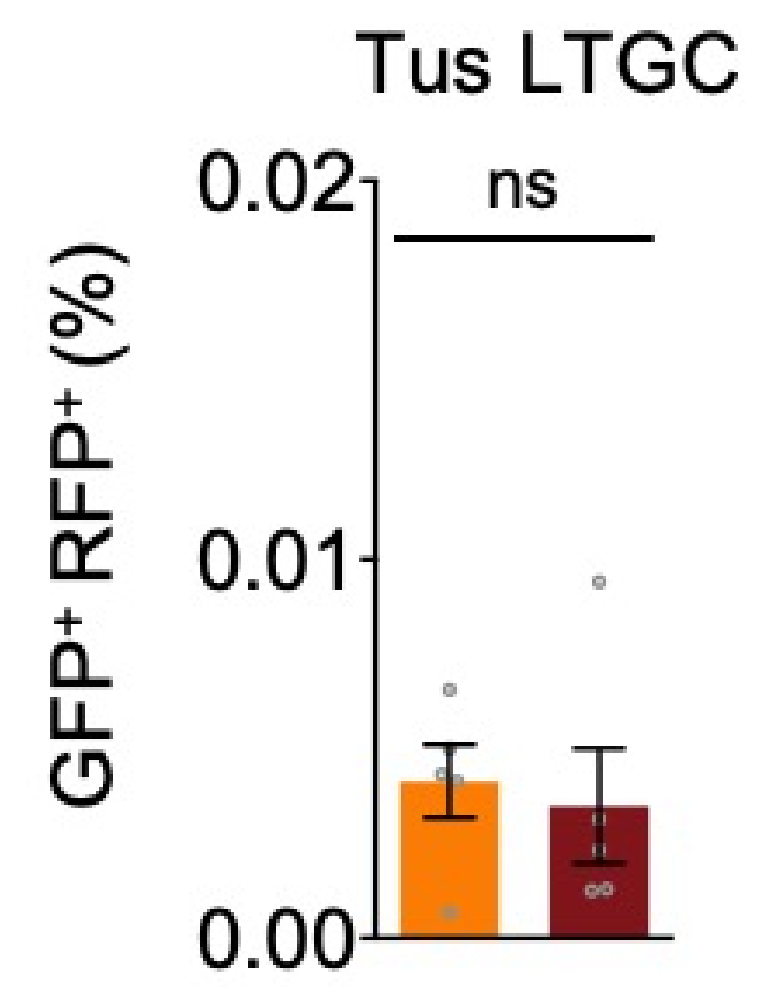

SIX4: +1+ +1-

I-Scel LTGC

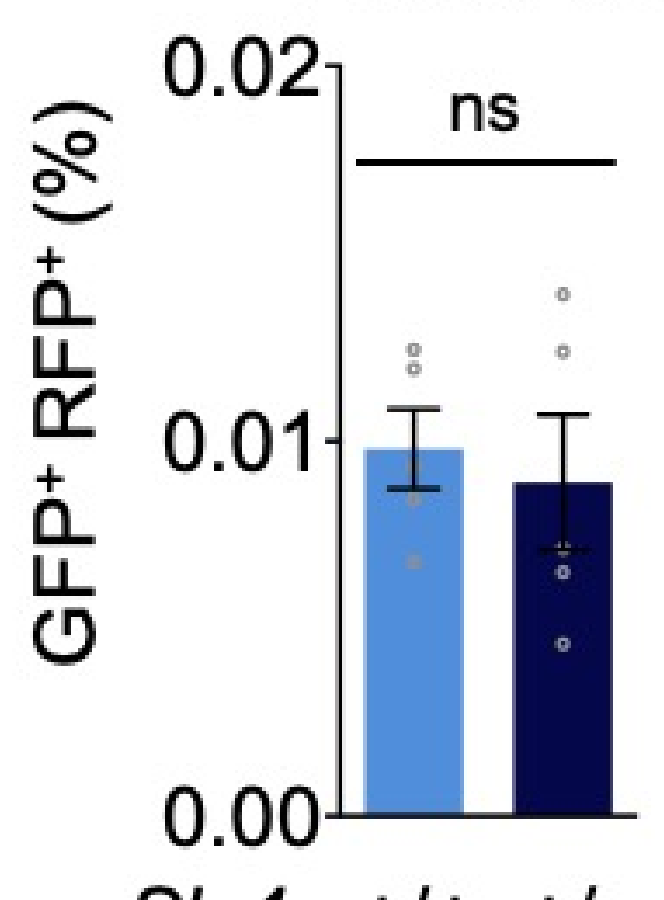

Slx4: +1+ +1-

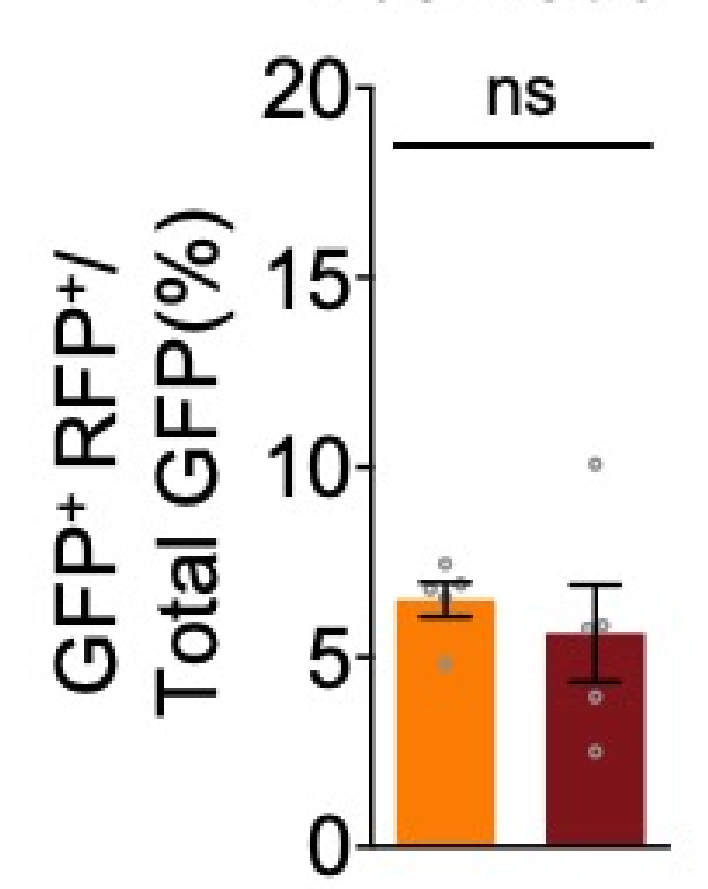

S/x4: +/+ +1-

I-Scel Ratio

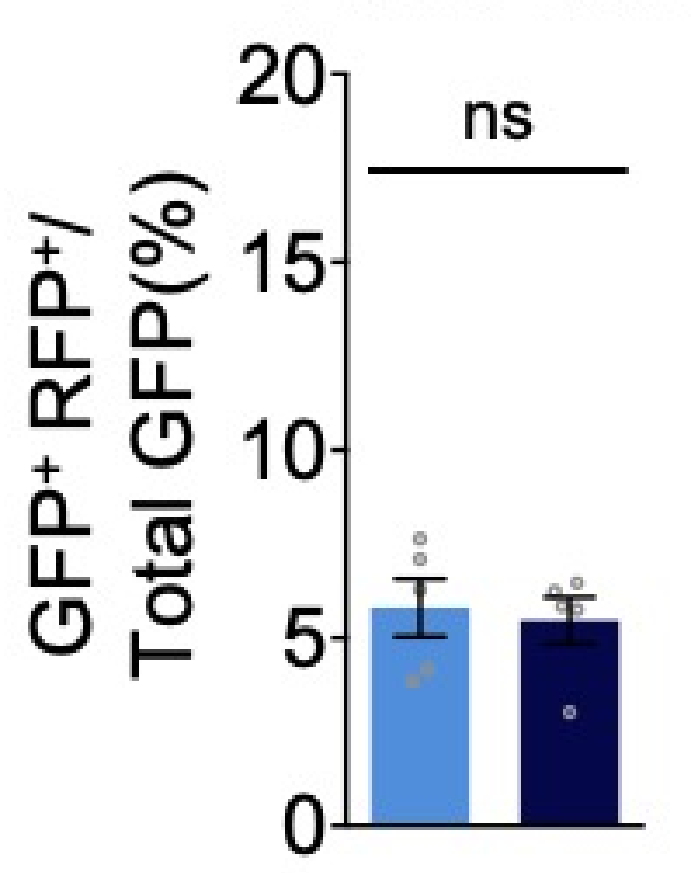

SIx4: $+1++1-$ 


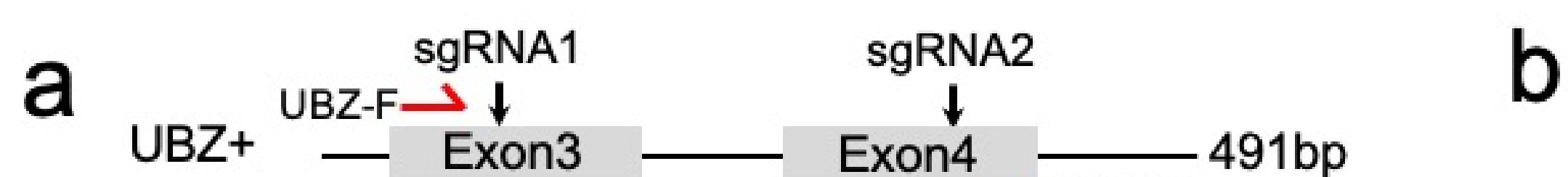

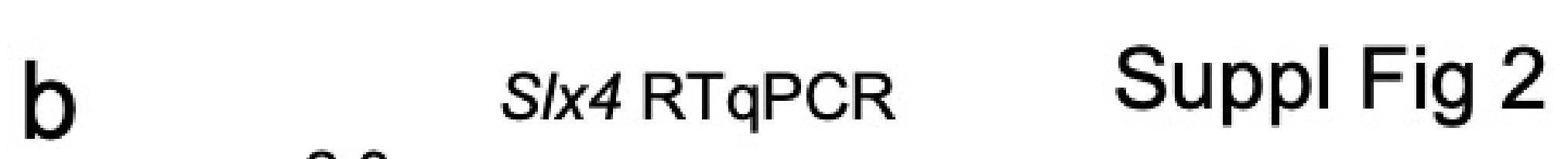

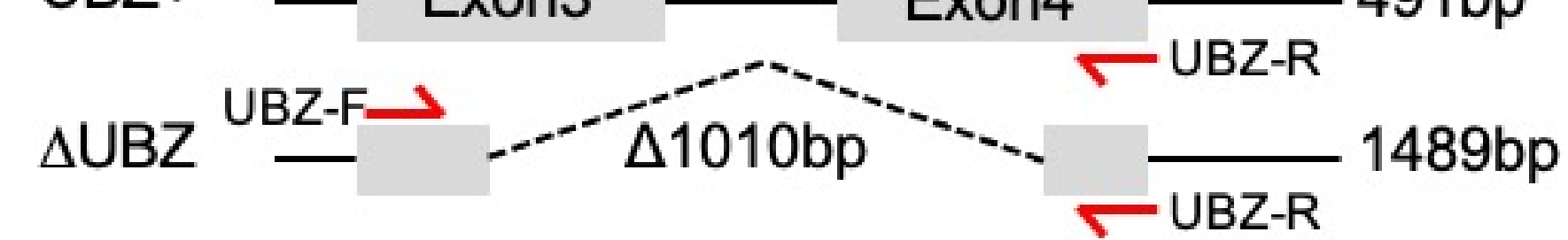

$1.5 \mathrm{~kb}-=-+\cdots=$

$0.5 \mathrm{~kb}-\quad-1--$

SIX4: $\frac{72544467181}{+/-} \frac{125111820}{\Delta U B Z /-}$

5' CACCATGACAGCCTGCTTCAGGCTGTGAGG 3'

3' GTGGTACTGTCGGACGAAGTCCGACACTCC 5' $\begin{array}{lllllllllll}\text { H } & H & \text { D } & \text { S } & \text { L } & \text { L } & \text { Q } & \text { A } & \text { V } & \text { R }\end{array}$

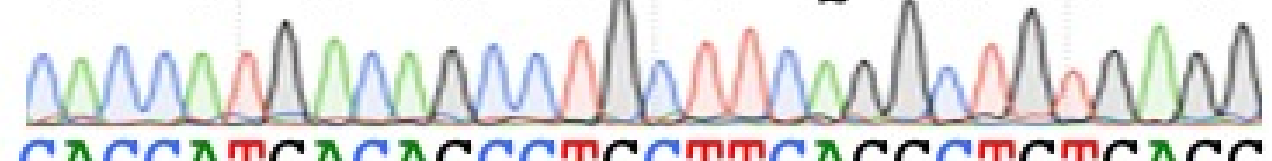
CACCATGACAGCCTGCTTCAGGCTGTGAGG
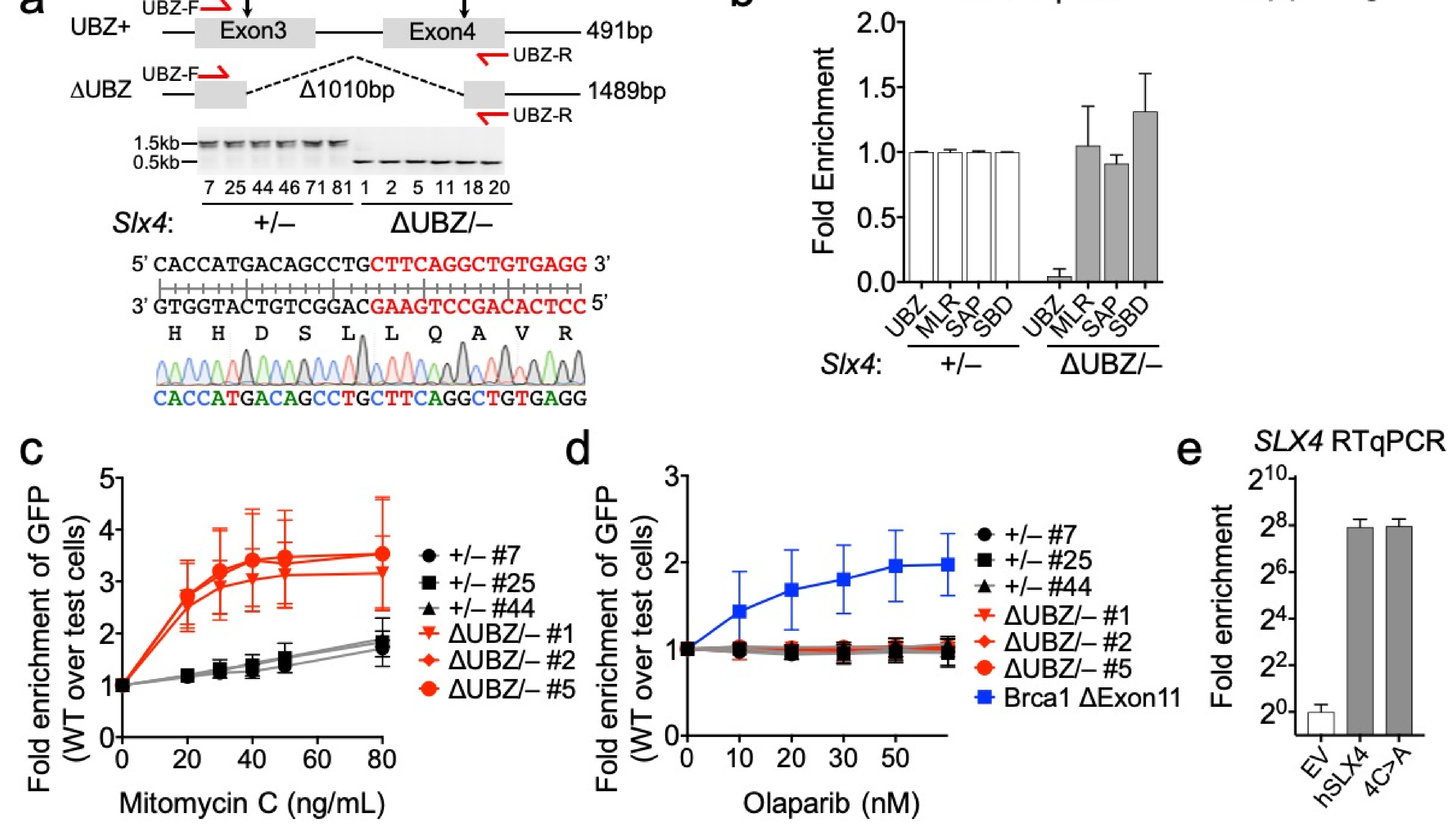

f
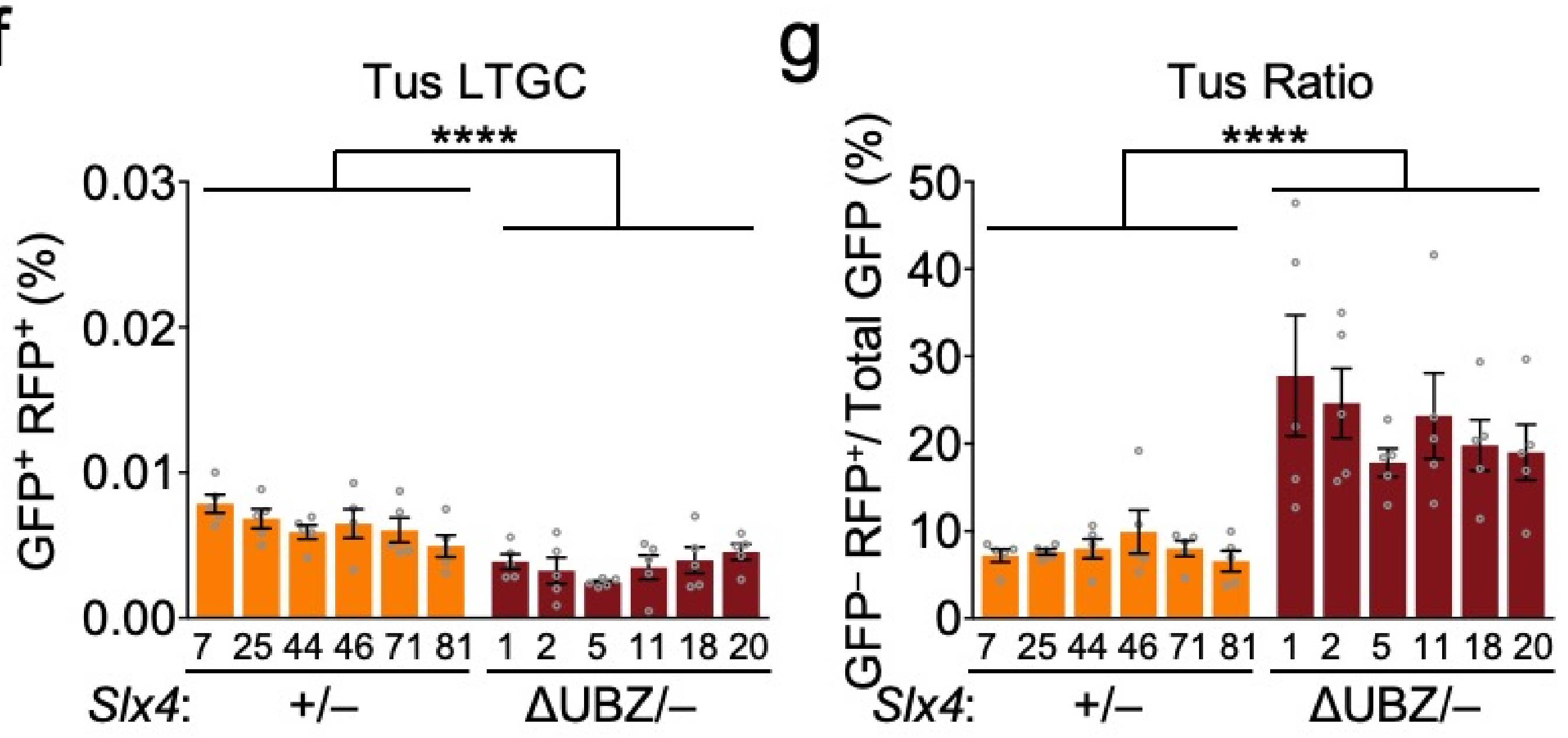

$\mathrm{h}$

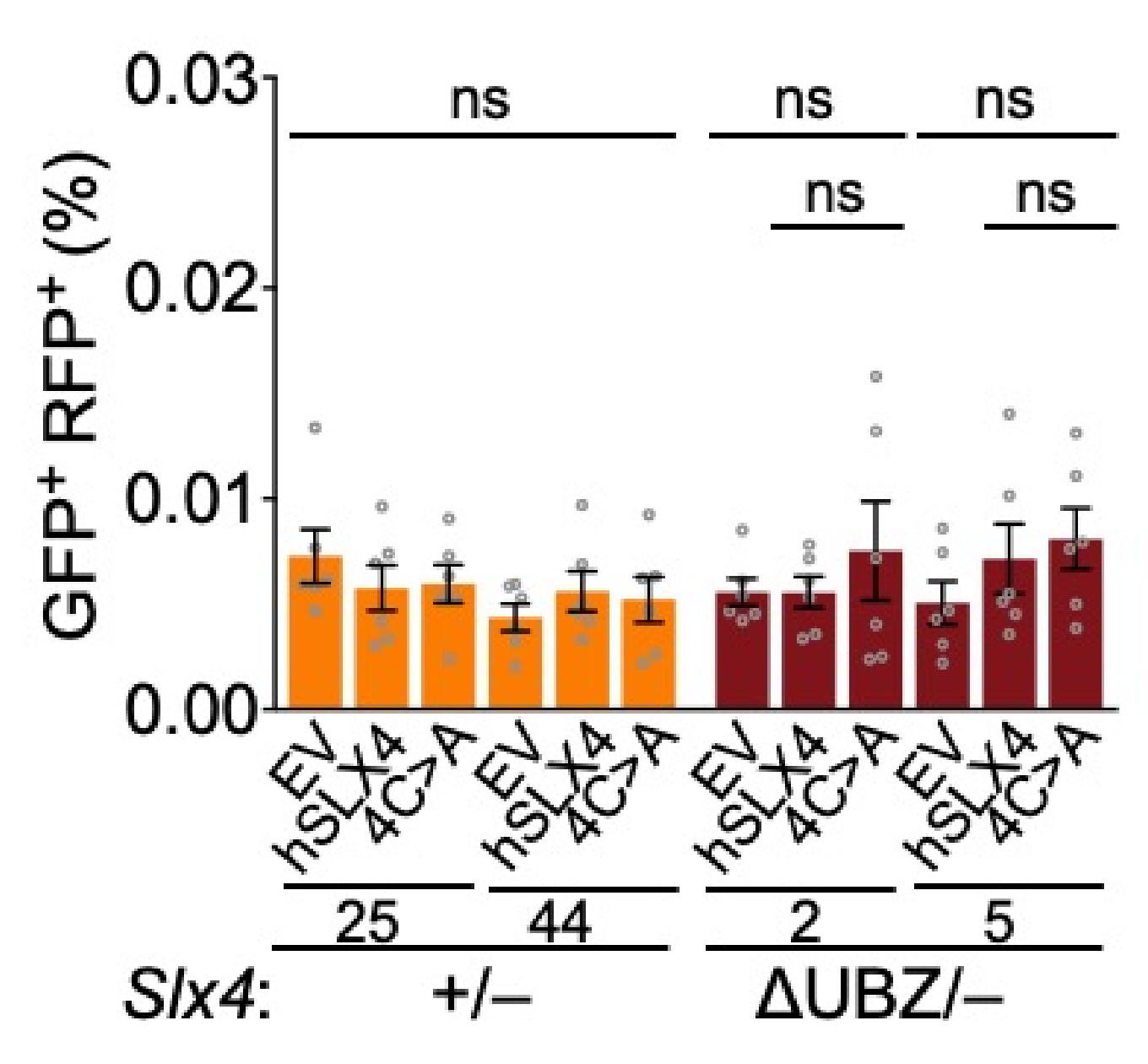

i
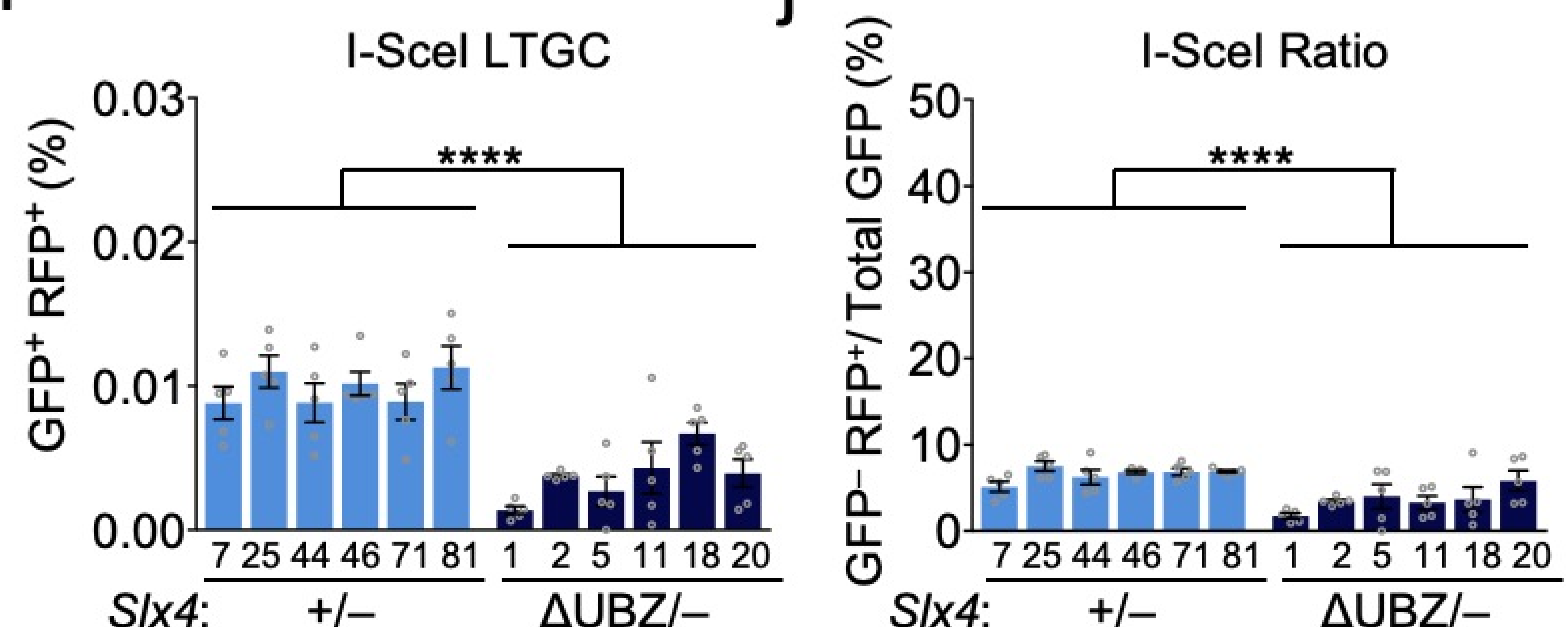

k

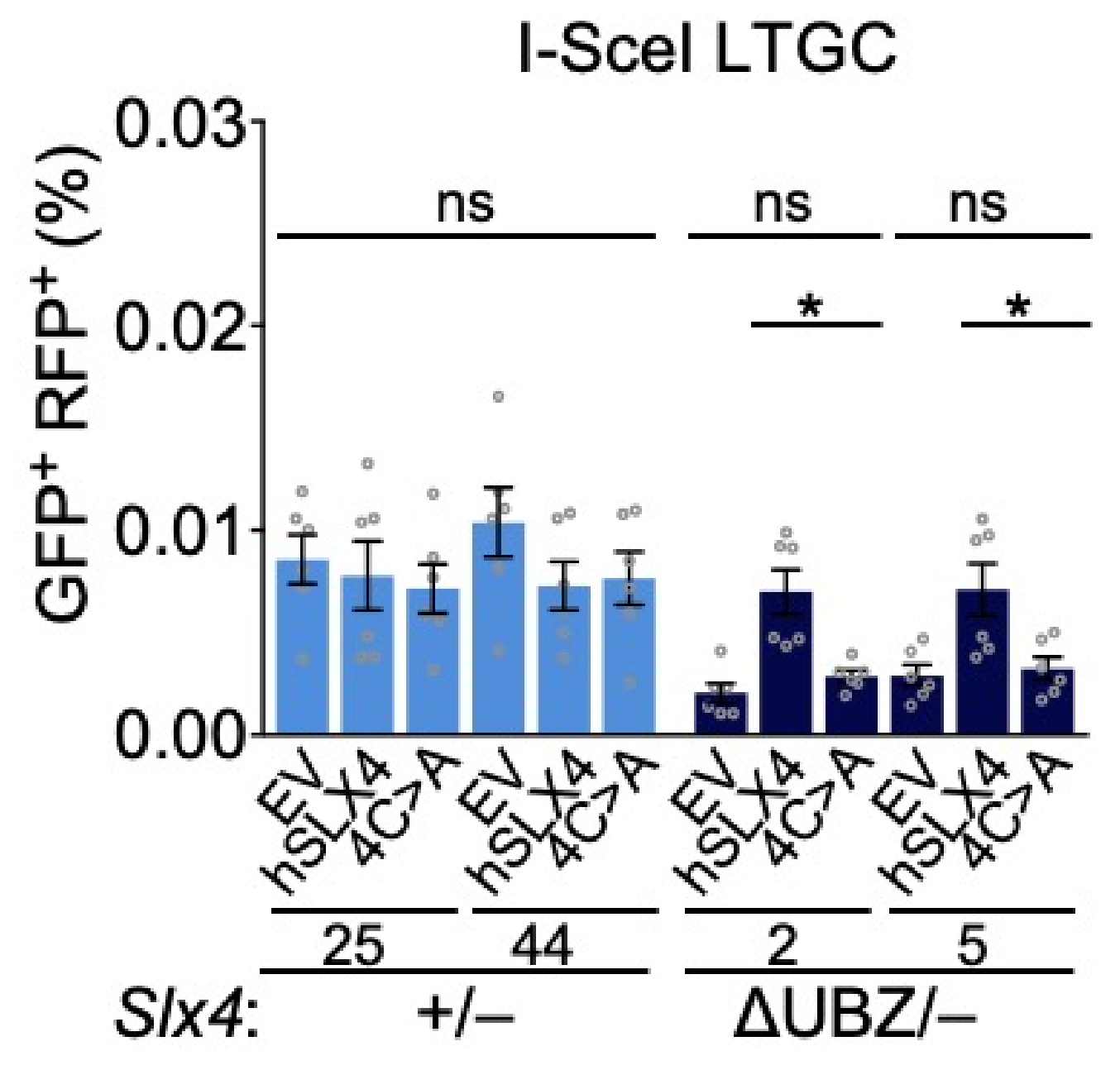




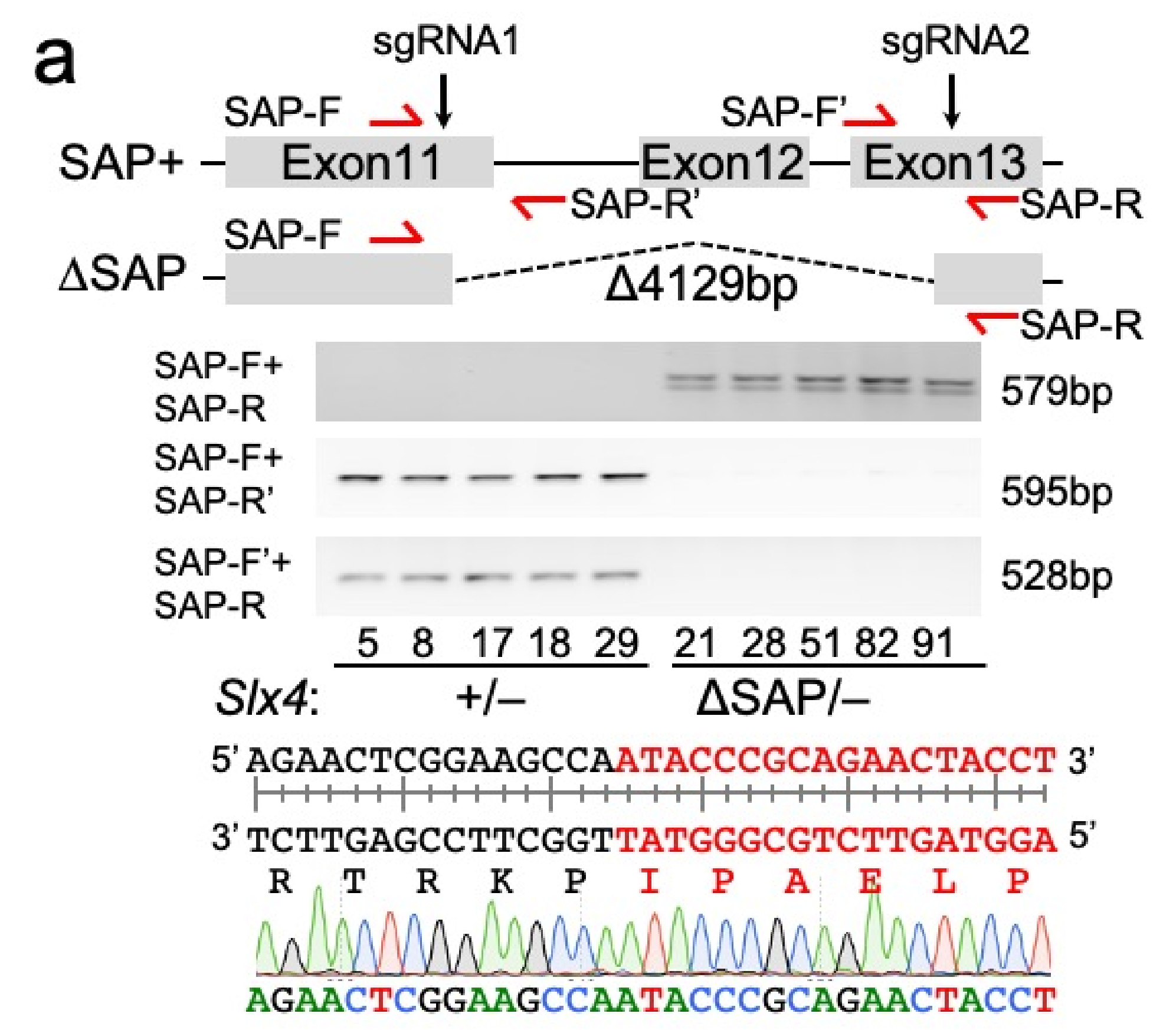

b $\quad$ Suppl Fig 3

C

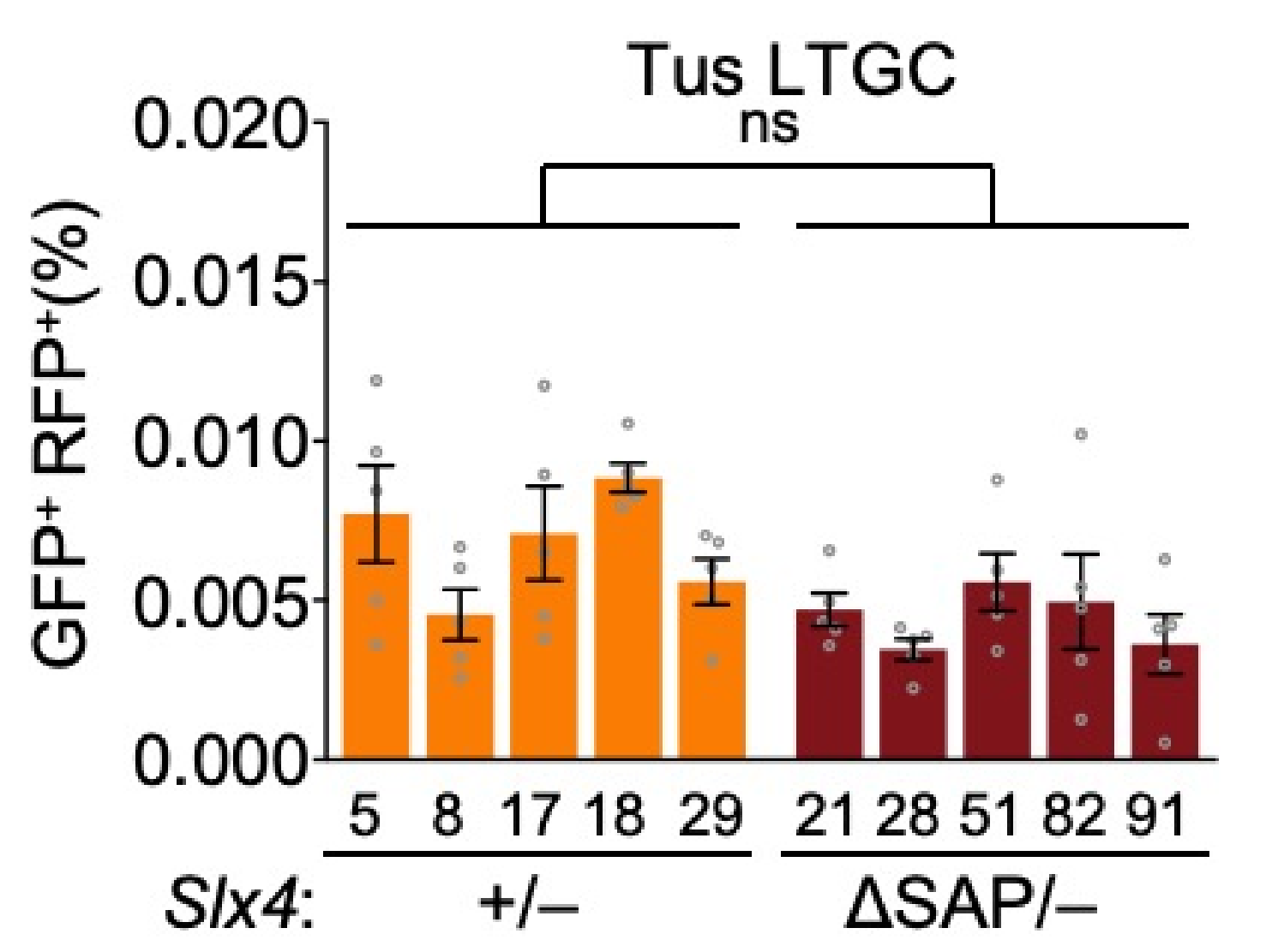

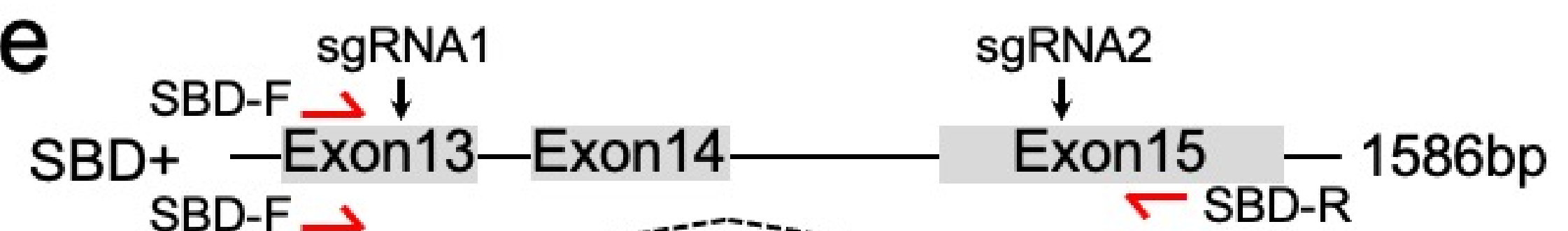
$\Delta \mathrm{SBD}-\ldots-\mathrm{SBD}-\mathrm{R} 550 \mathrm{bp}$

S/x4: $\frac{121617224451}{+/-} \frac{-\overline{1529} 31416282}{\Delta \mathrm{SBD} /-}$

5' GAACTACCTTGTCGACAGGCCTCCACCCAGTAG 3'

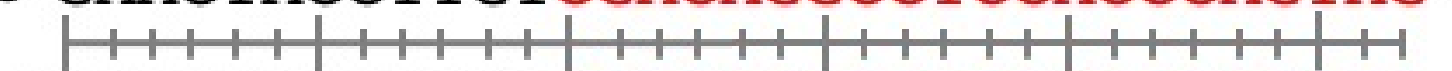

3' CTTGATGGAACAGCTGTCCGGAGGTGGGTCATC 5'

$\begin{array}{llllllllllll}\mathrm{E} & \mathrm{L} & \mathrm{P} & \mathrm{C} & \mathrm{R} & \mathrm{Q} & \mathrm{A} & \mathrm{S} & \mathrm{T} & \mathrm{Q} & \mathrm{STOP}\end{array}$

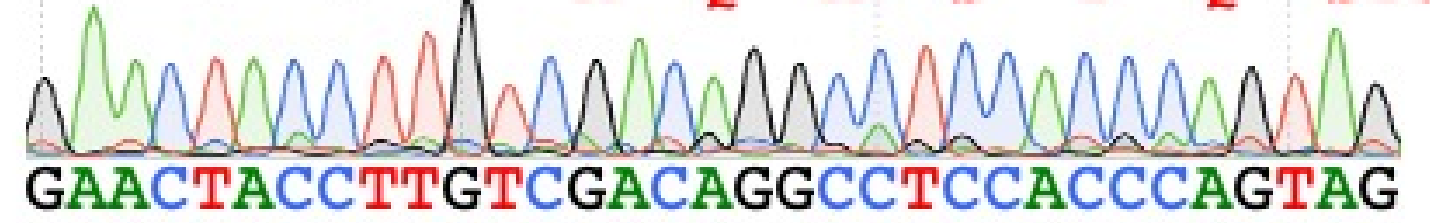

g

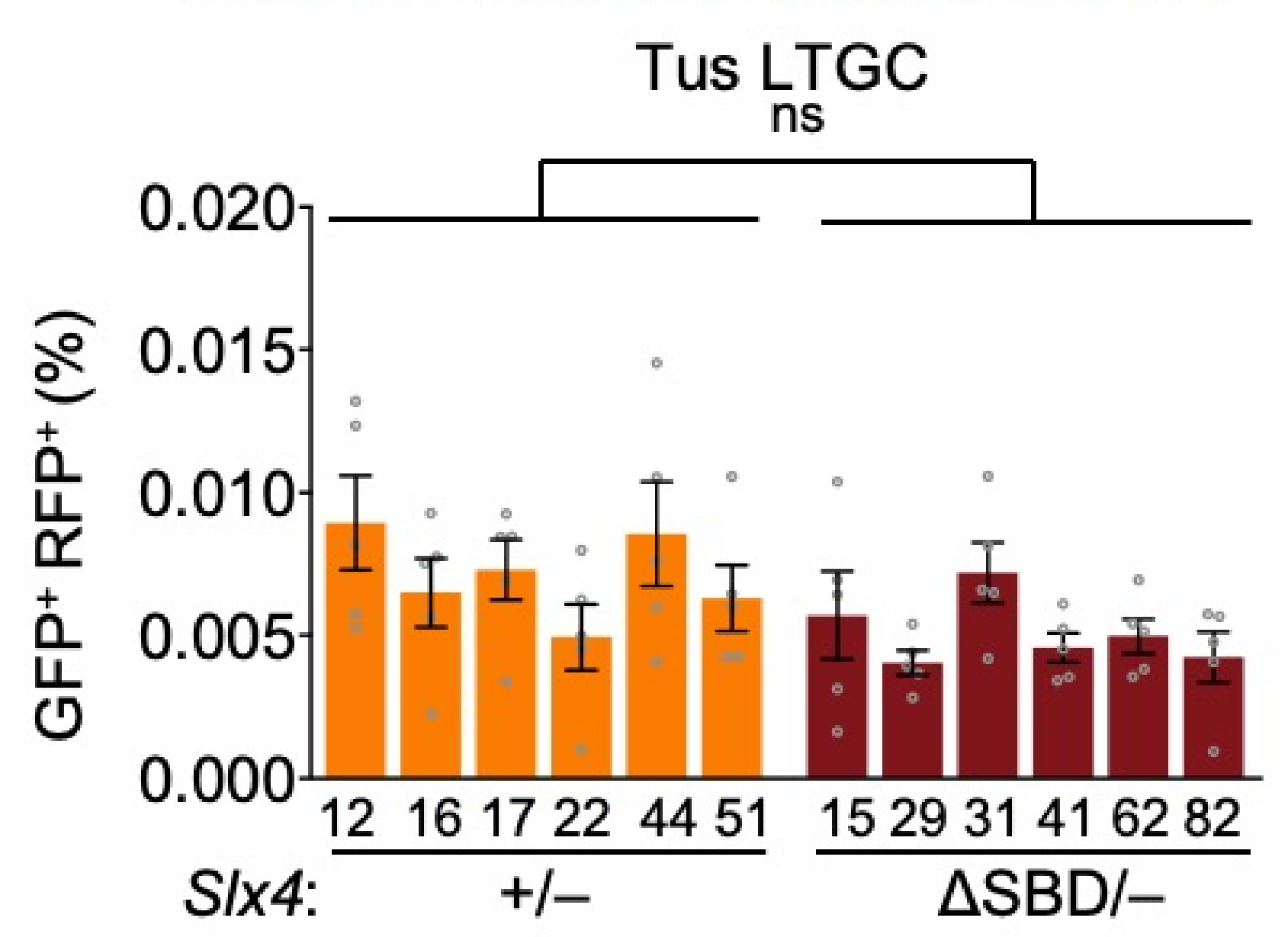

$\mathrm{h}$

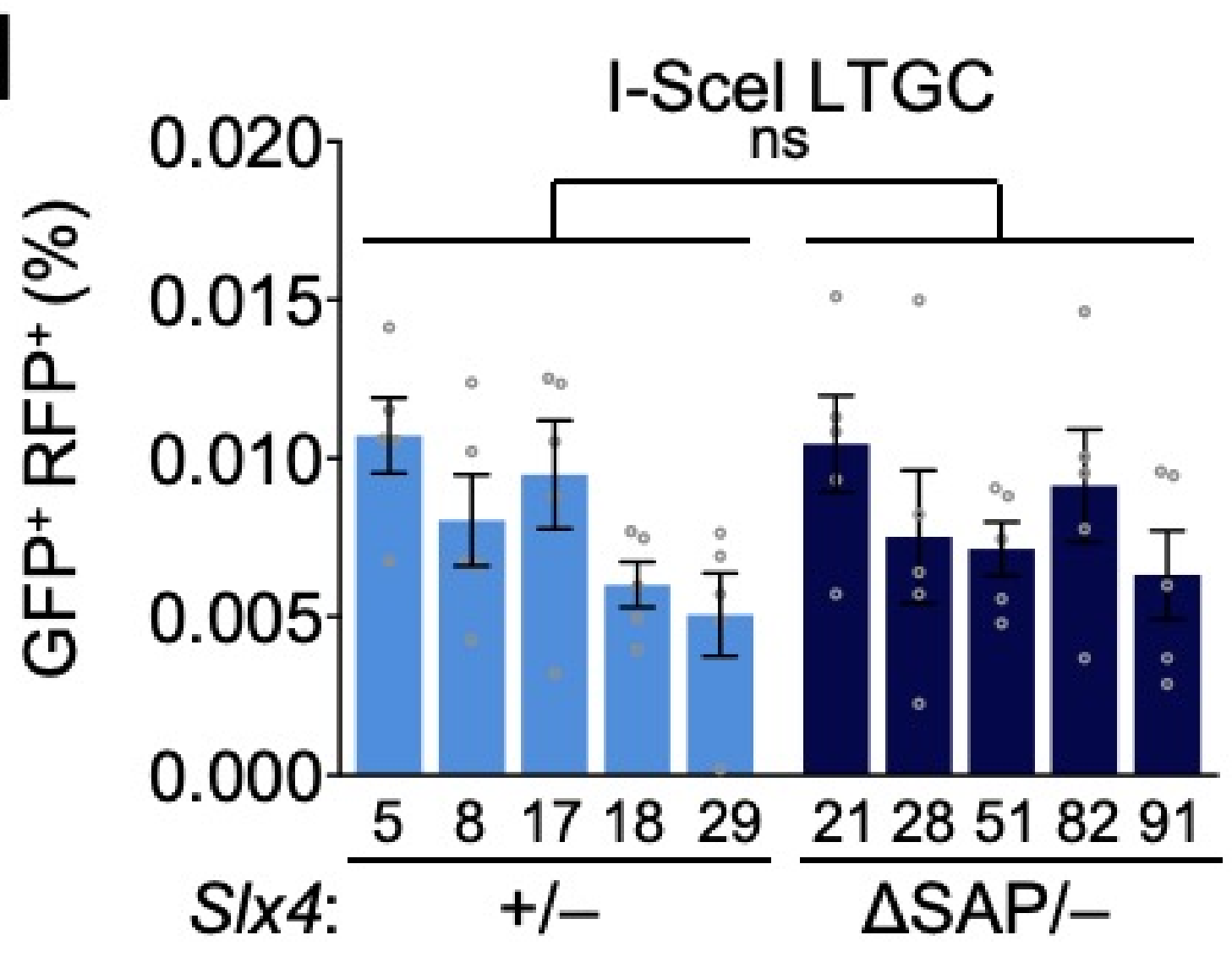

f
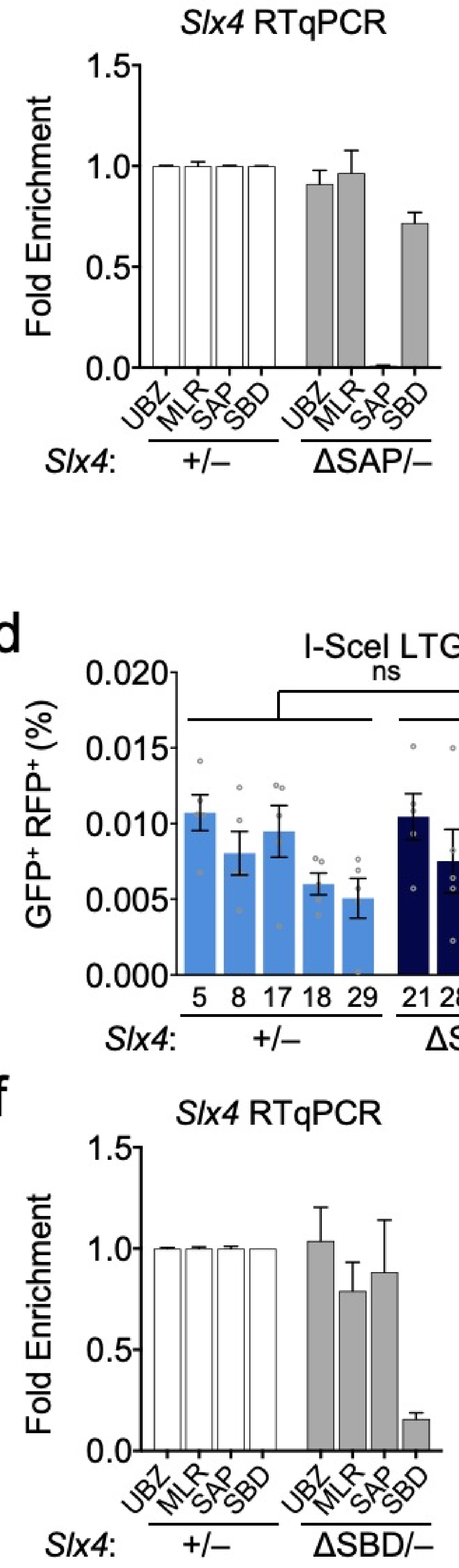

I-Scel LTGC

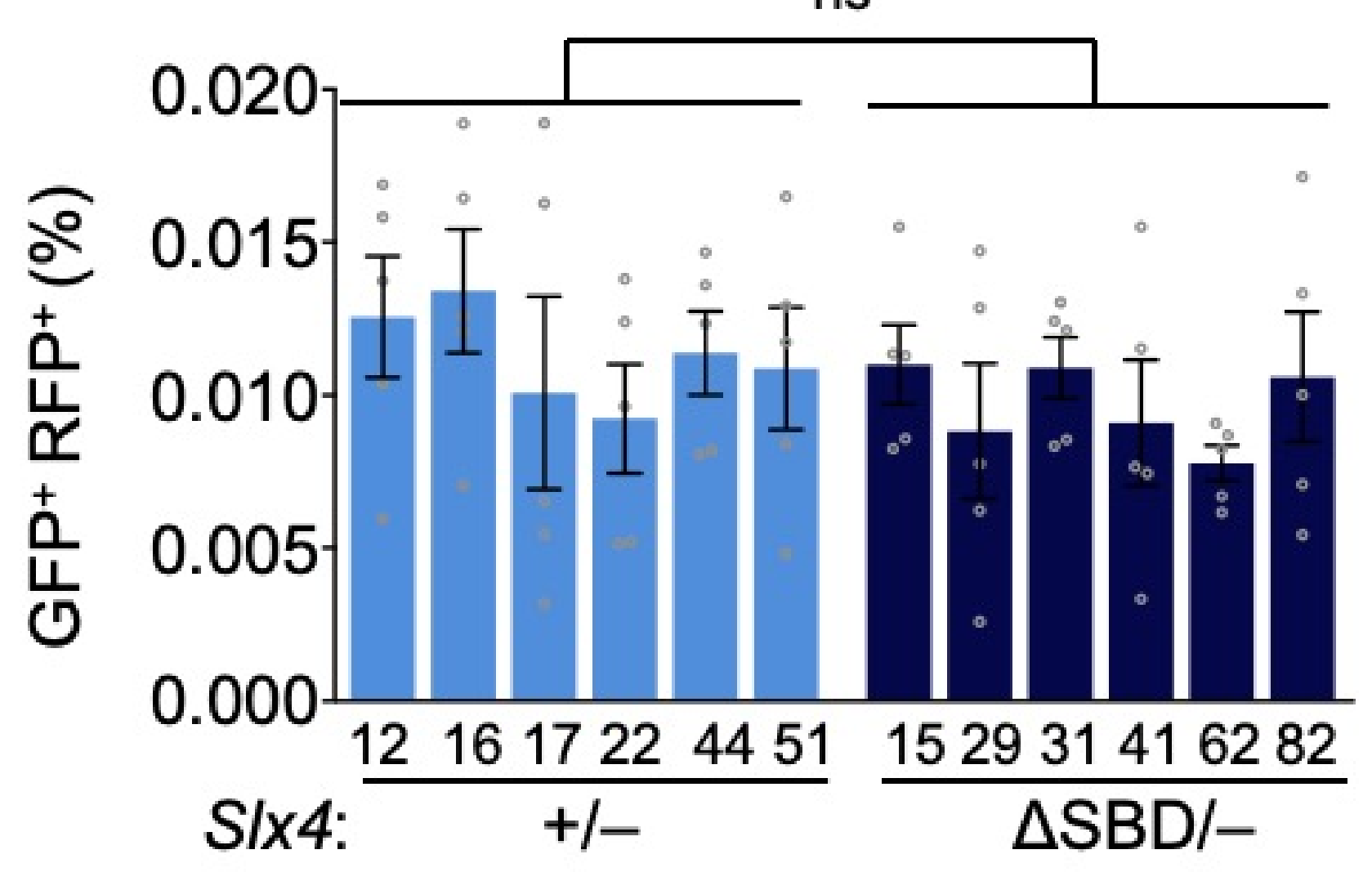


a

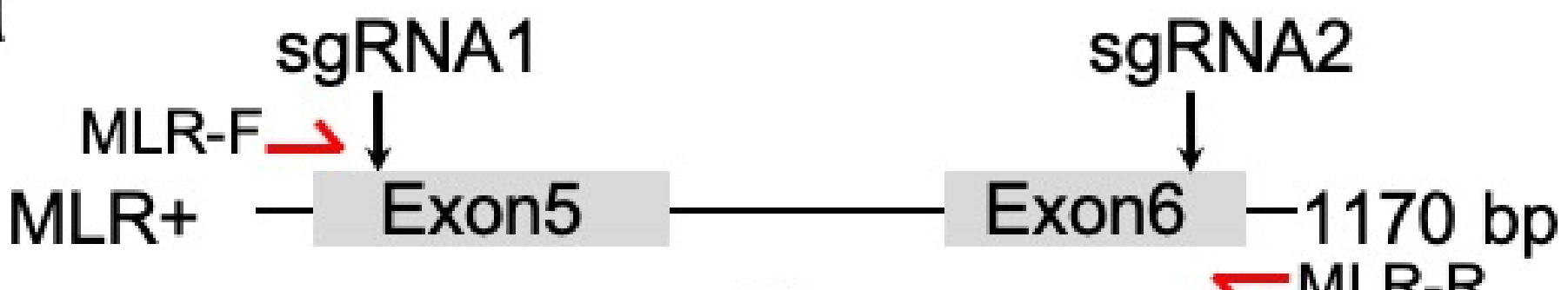

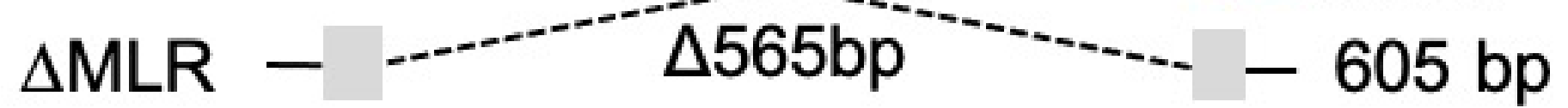

$1.5 \mathrm{~kb}-$

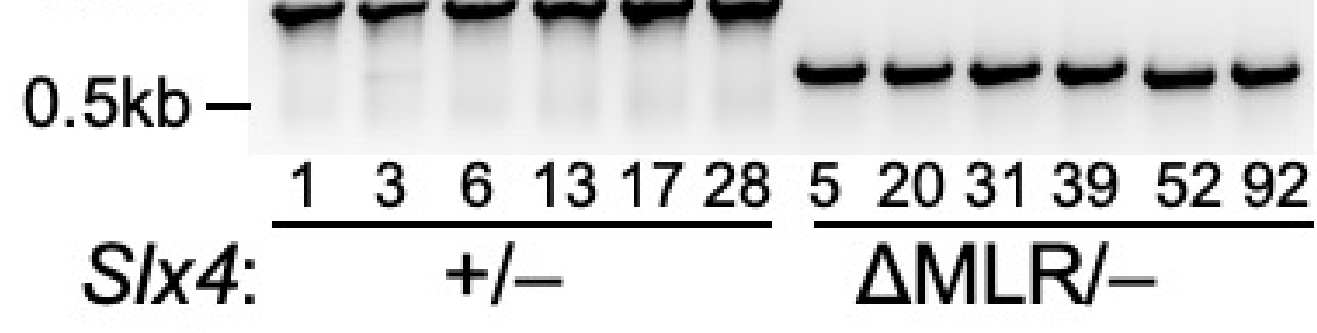

5'AgGGAGCCAAGGATTGTGTCCCAATGC 3' 3'TCCCTCGGTTCCTACAGGGTTACGGGA 5'

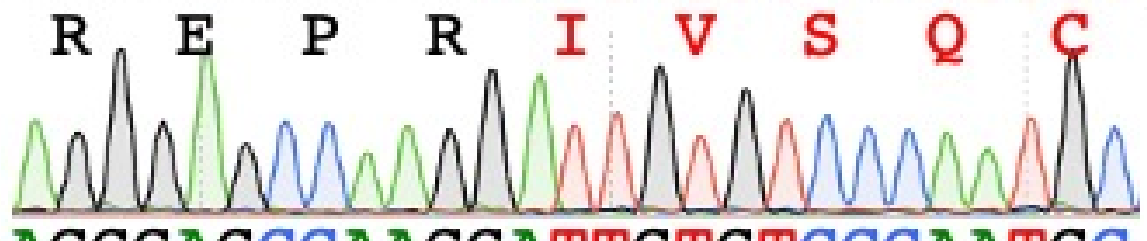

AGGGAGCCAAGGATTGTGTCCCAATGC

C

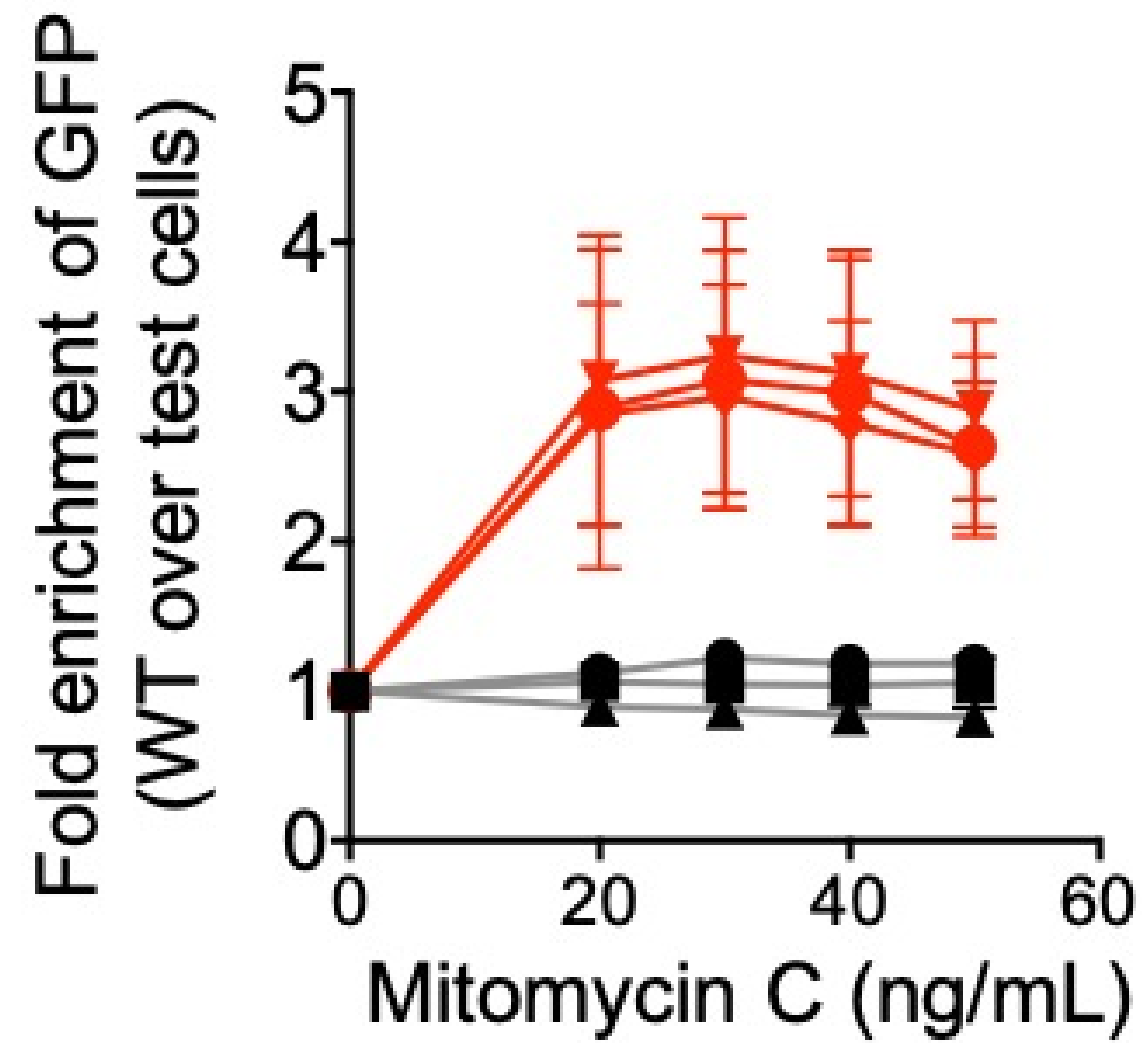

e

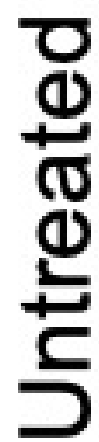

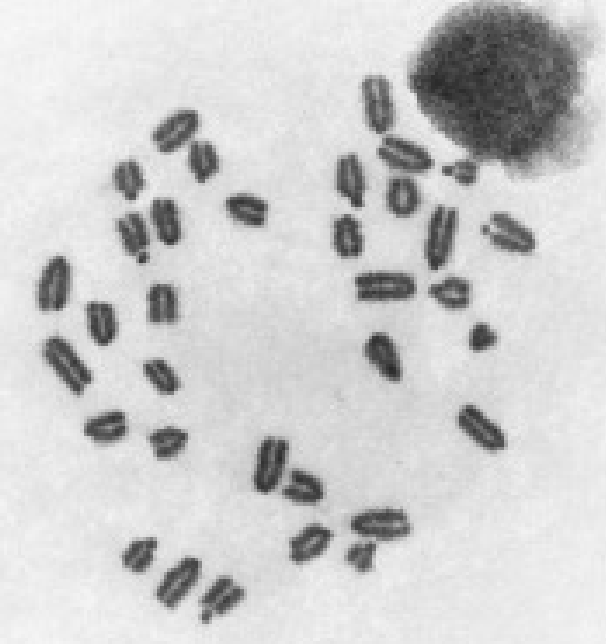

$\Delta \mathrm{SAP} /-$ $\sum$

$\Delta \mathrm{SAP} /-$
- +1- \#13

$+1-\# 17$

- +1- \#28

₹ $\triangle \mathrm{MLR} /-\# 5$

$\triangle M L R /-\# 31$
* $\triangle \mathrm{MLR} /-\# 20$

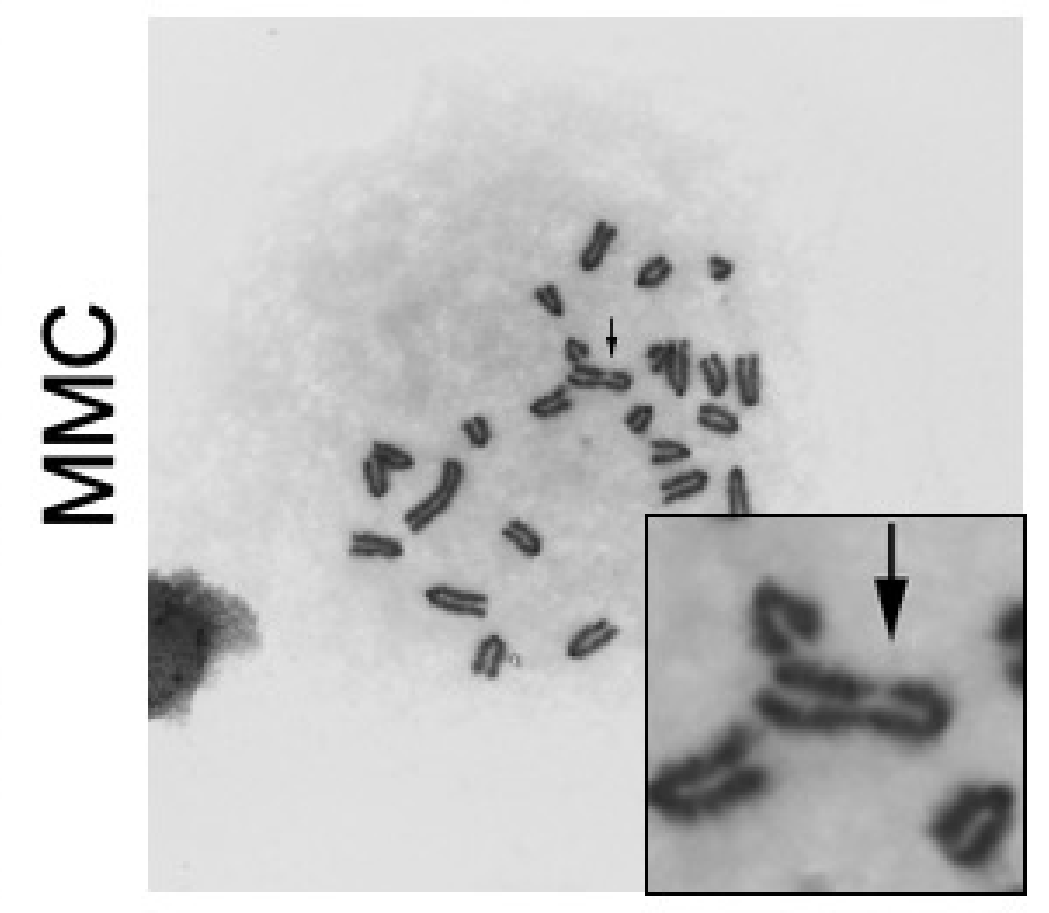

d
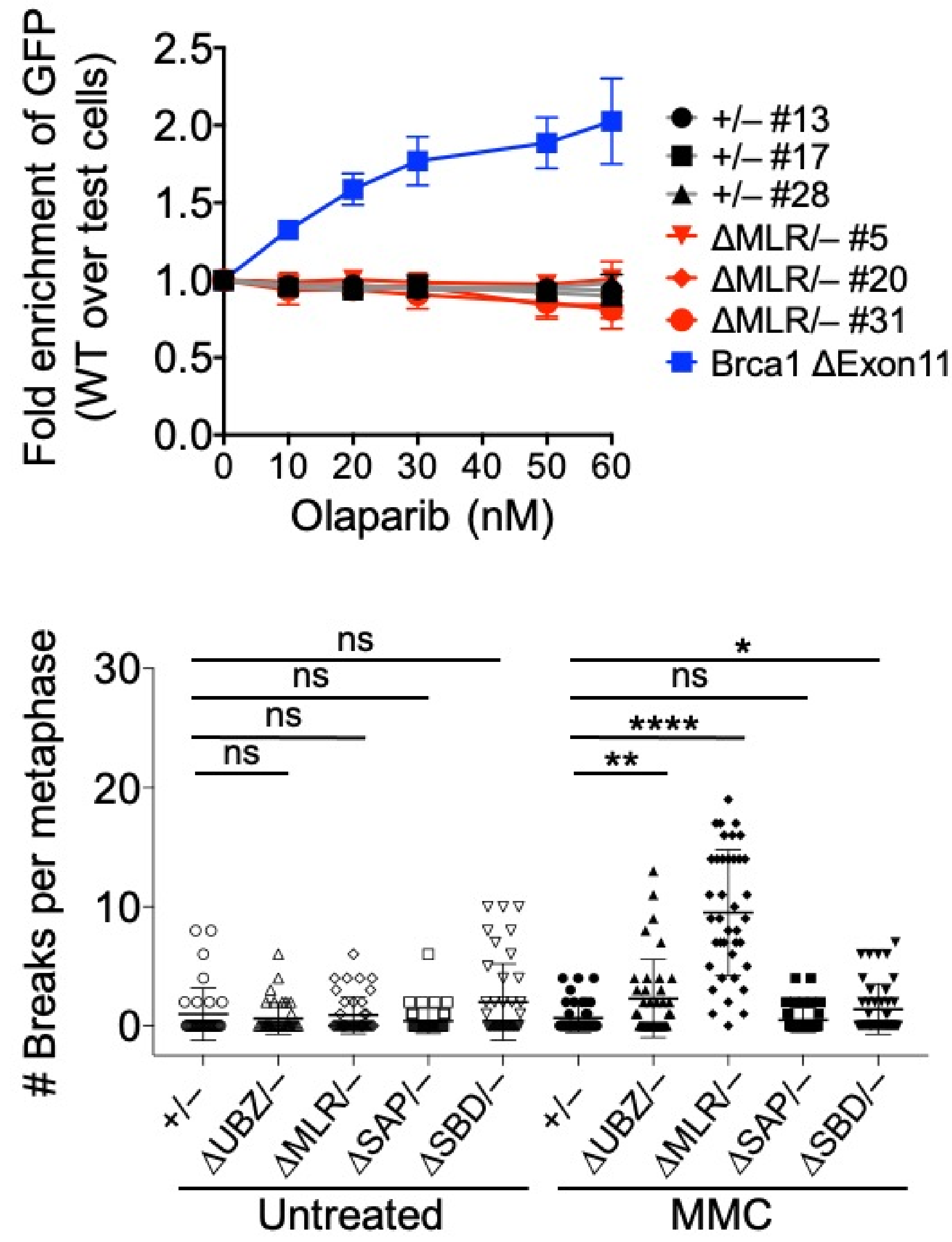

g

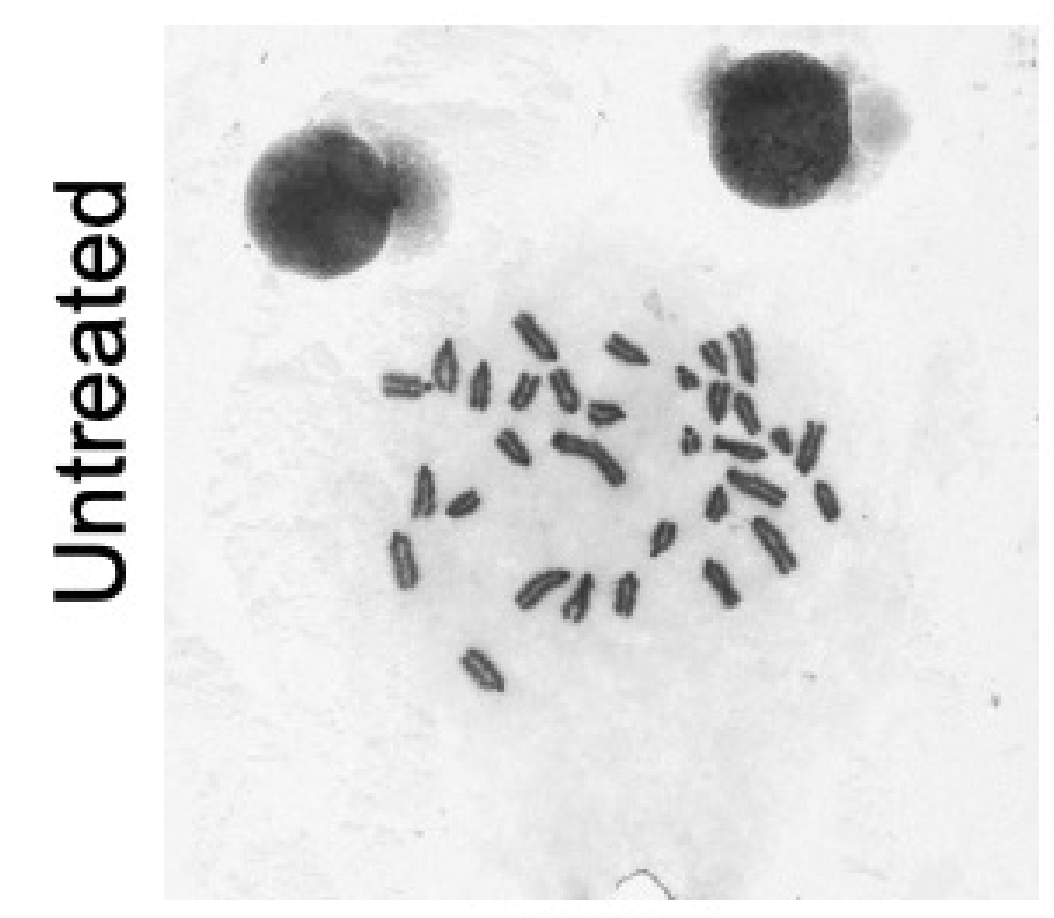

$\Delta \mathrm{SBD} /-$

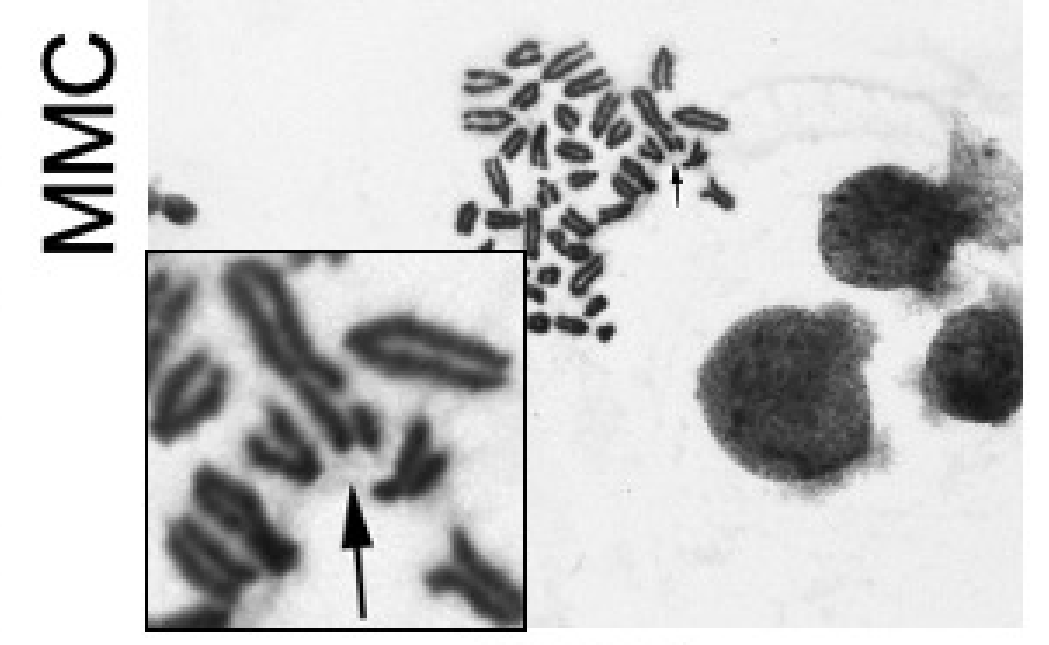

$\Delta \mathrm{SBD} /-$

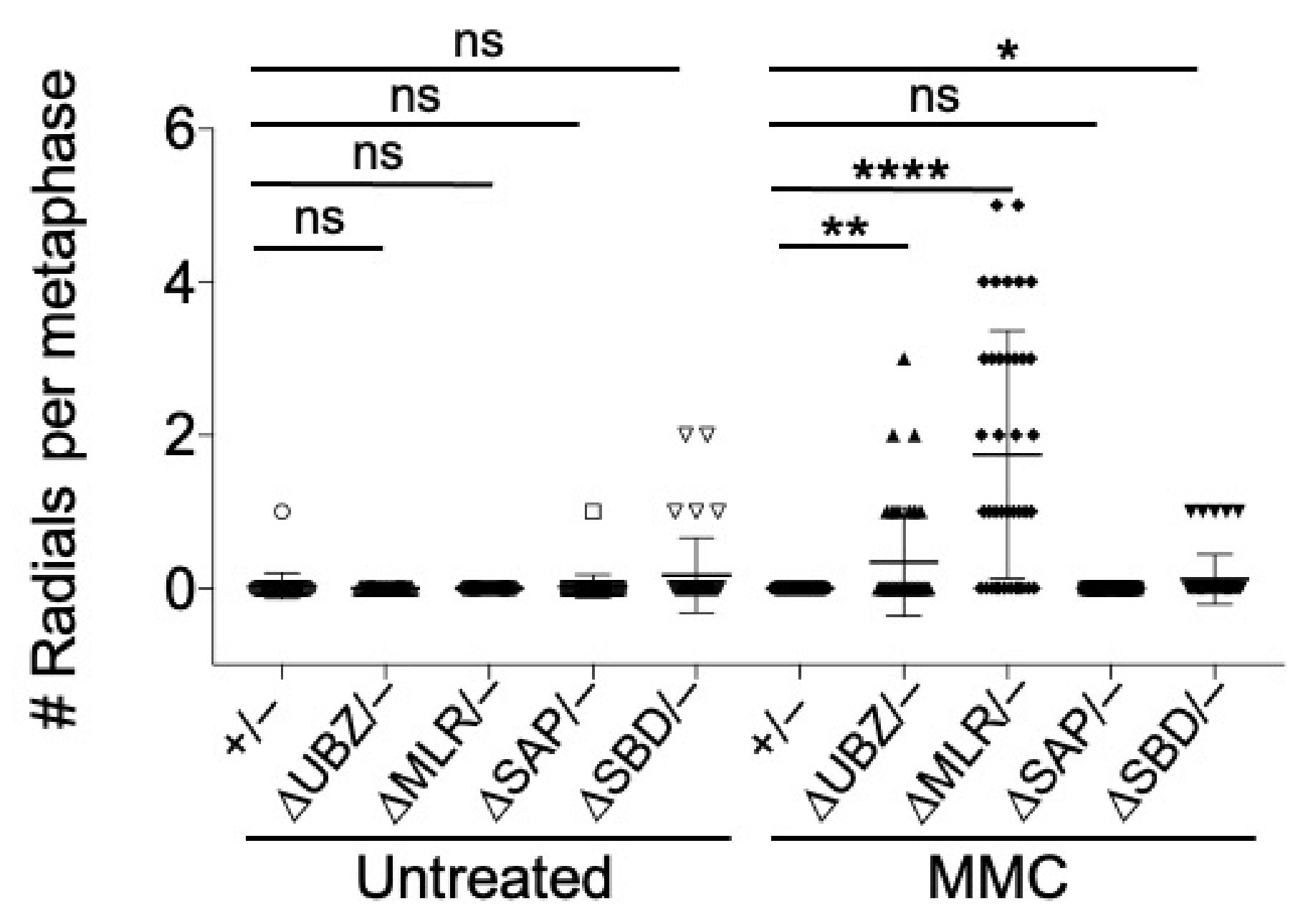

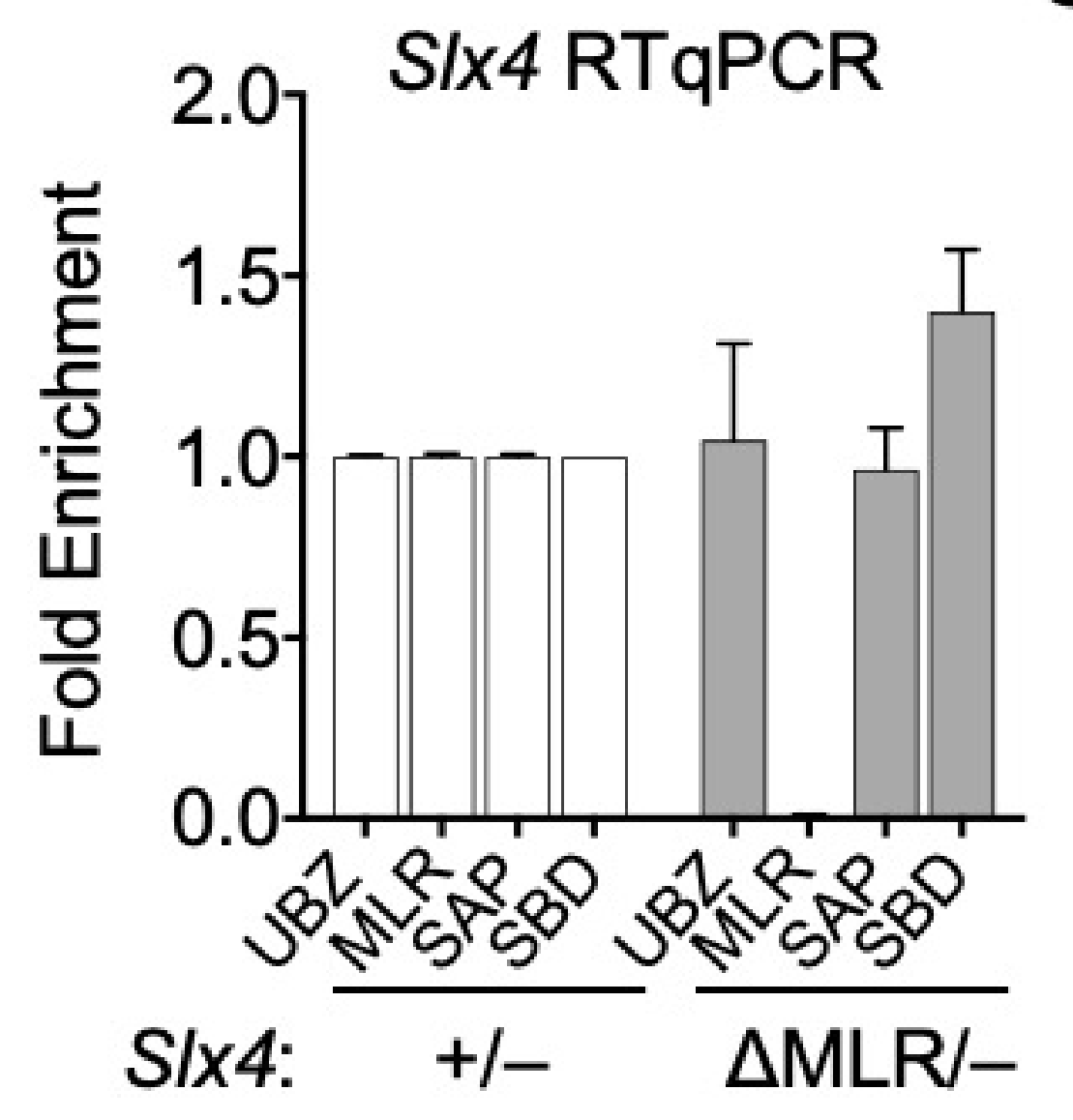


a

Tus LTGC

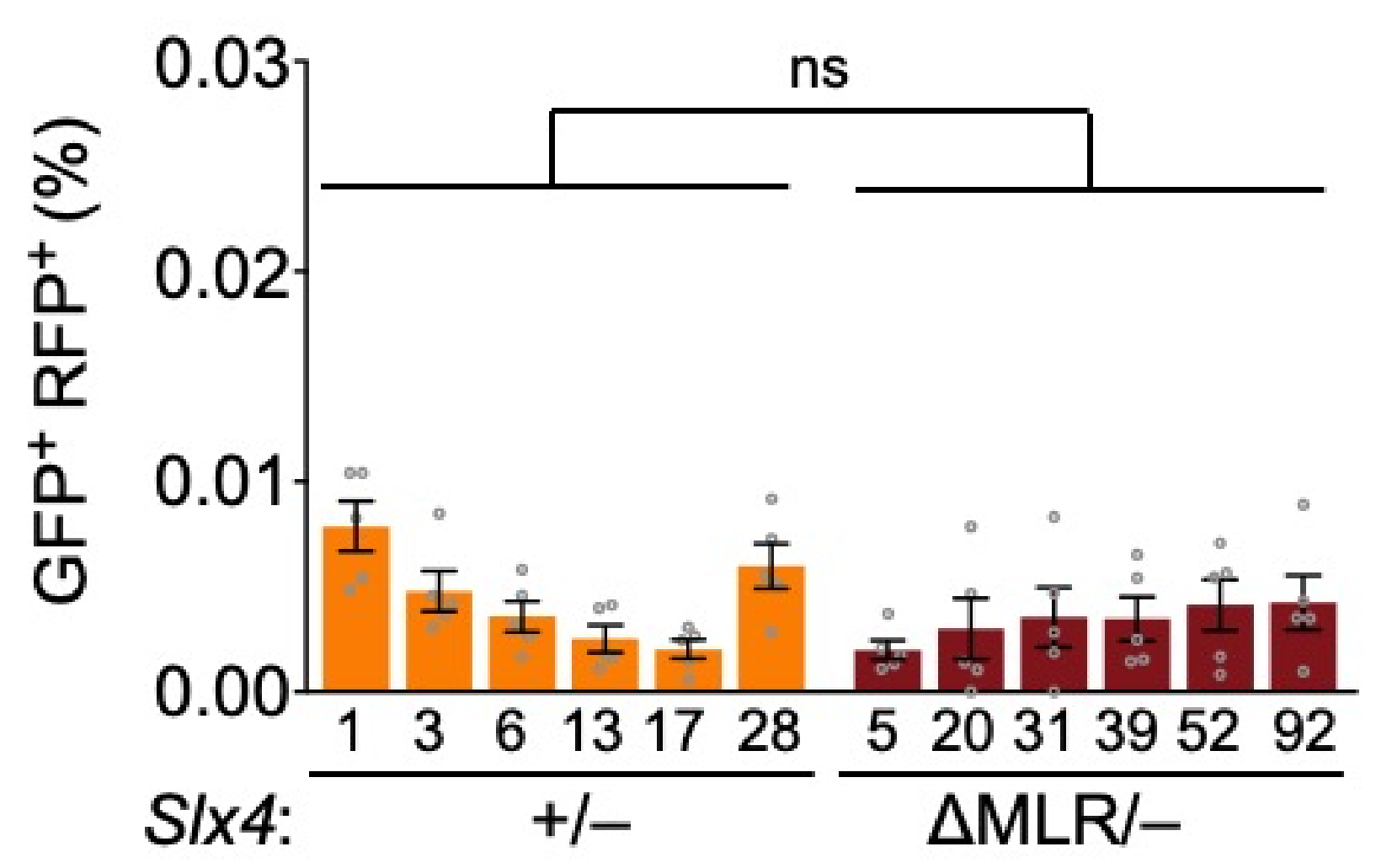

C

Tus LTGC

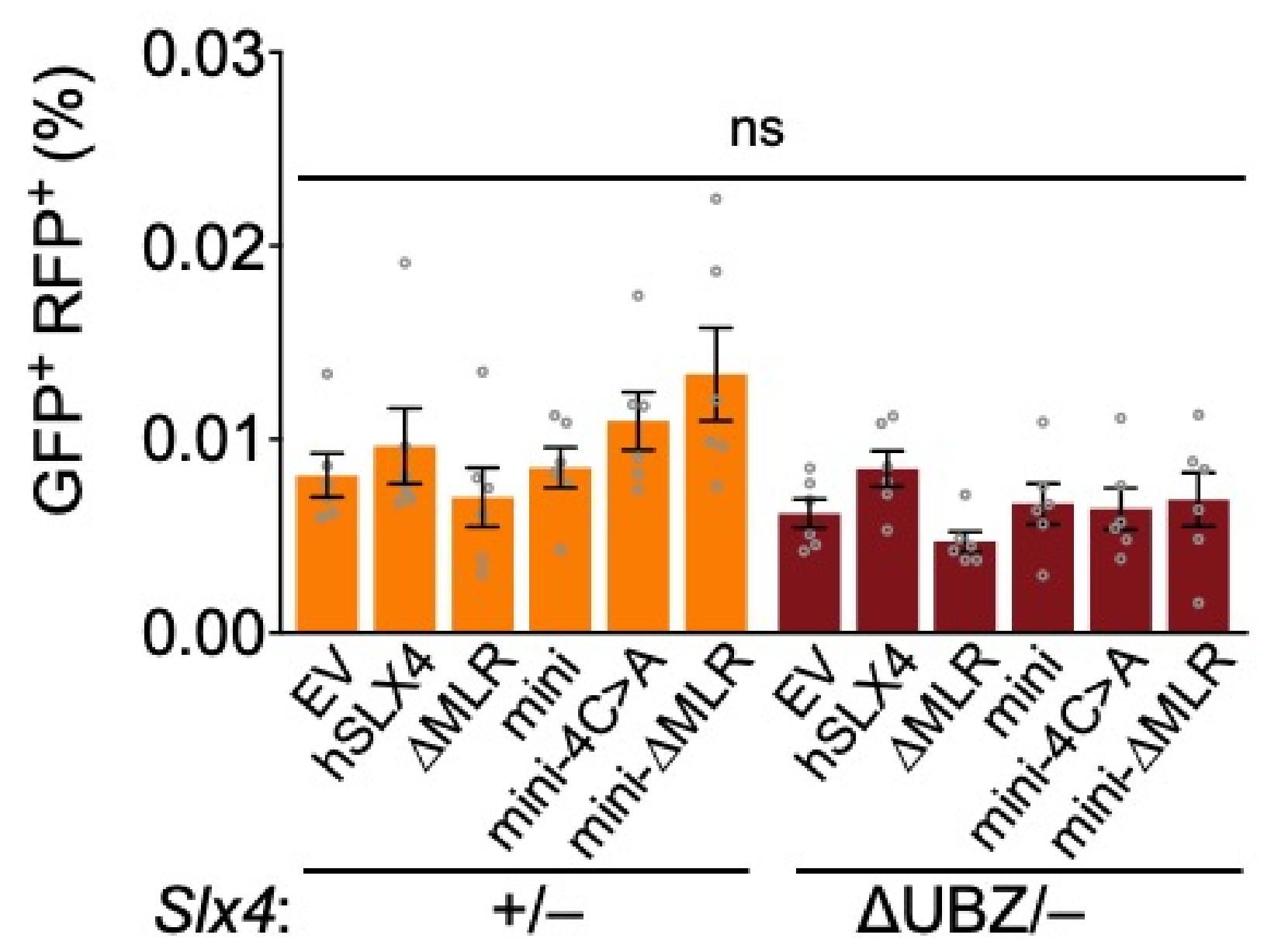

b

Suppl Fig 5

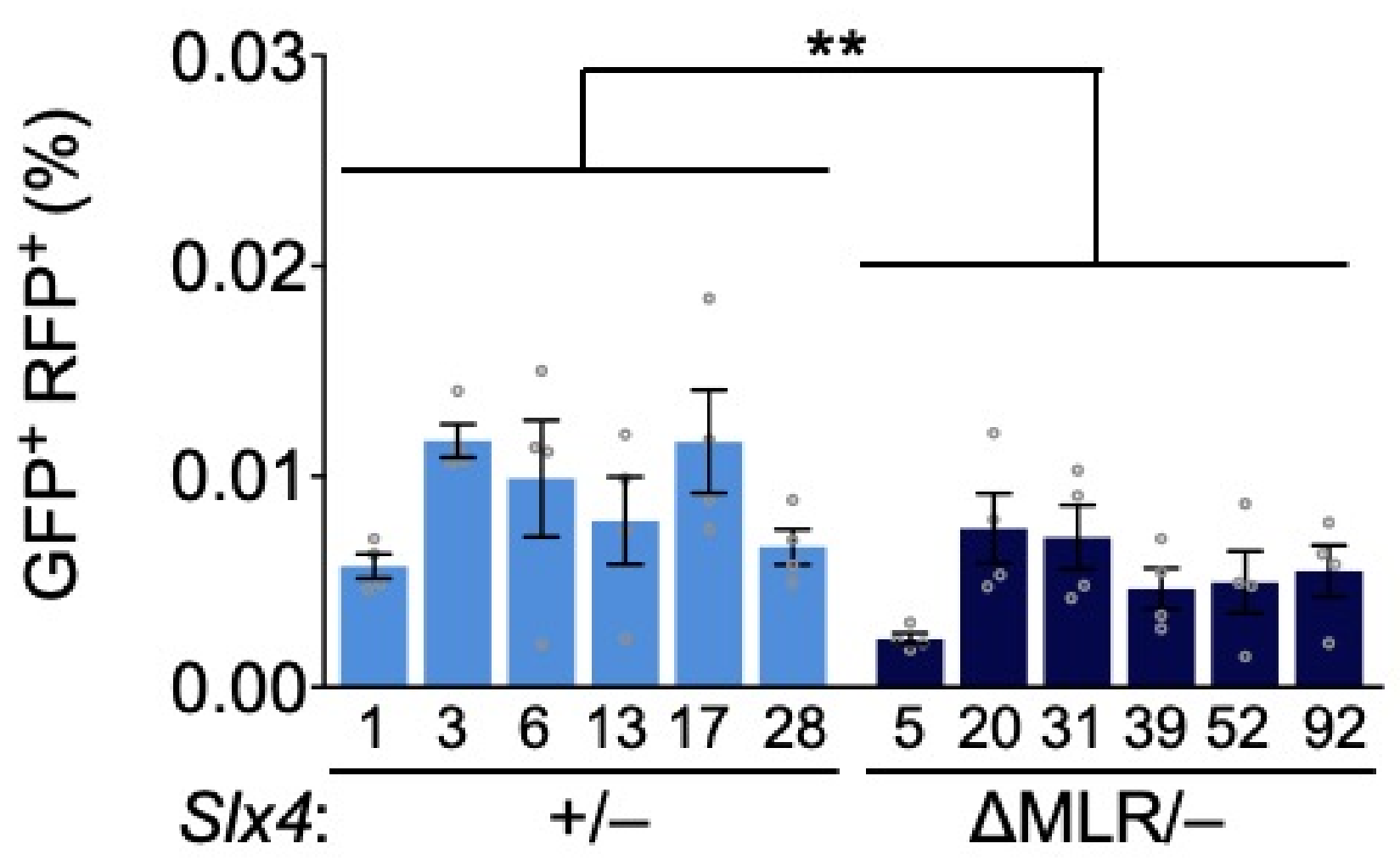

d

I-Scel LTGC

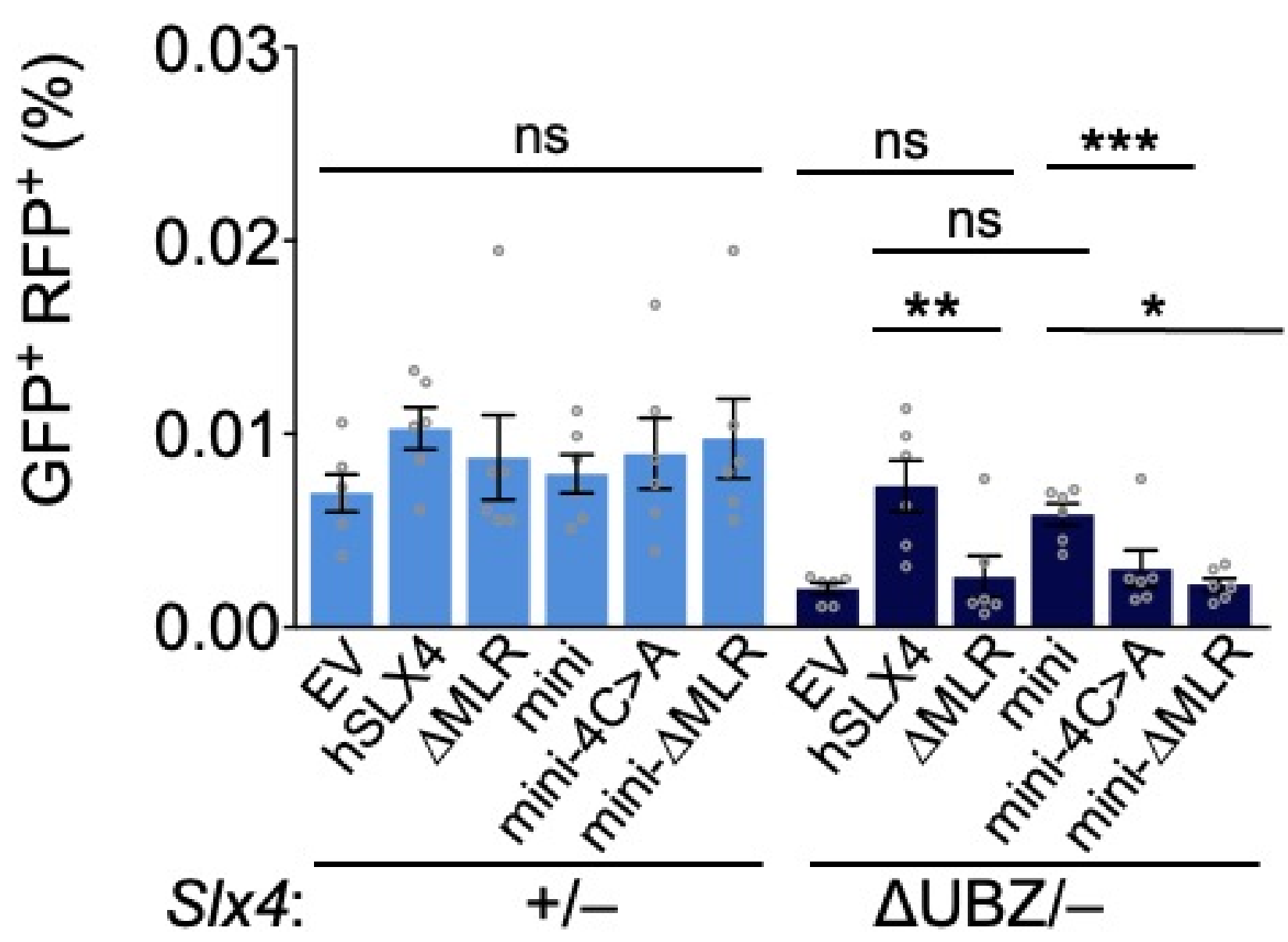


